\title{
TRACHEAL TISSUE ENGINEERING
}

\author{
by \\ C.M. Hobson \\ BA in Physics \& Biochemistry, Franklin \& Marshal College, 2006 \\ MS in Materials Engineering, Drexel University, 2008 \\ Submitted to the Graduate Faculty of \\ the Swanson School of Engineering in partial fulfillment \\ of the requirements for the degree of \\ Doctor of Philosophy
}

University of Pittsburgh

2014 


\section{UNIVERSITY OF PITTSBURGH SWANSON SCHOOL OF ENGINEERING}

This dissertation was presented

by

C.M. Hobson

It was defended on

March 19th 2014

and approved by

Peter D. Wearden, MD, PhD, Assistant Professor, Department of Surgery

Thomas W. Gilbert, PhD, Adjunct Professor, Department of Bioengineering

William R. Wagner, PhD, Professor, Department of Surgery, Bioengineering \& Chemical and Petroleum Engineering

Steven D. Abramowitch, PhD, Assistant Professor, Departments of Bioengineering and Obstetrics, Gynecology \& Reproductive Sciences

Alejandro Soto-Gutiérrez, MD, PhD, Assistant Professor, Department of Pathology \& Surgery

Dissertation Directors: Peter D. Wearden, MD, PhD, Assistant Professor, Department of Surgery,

Thomas W. Gilbert, PhD, Adjunct Professor, Department of Bioengineering 
Copyright (c) by C.M. Hobson

2014 


\title{
TRACHEAL TISSUE ENGINEERING
}

\author{
C.M. Hobson, PhD \\ University of Pittsburgh, 2014
}

Large airway defects pose a substantial problem to surgeons in both pediatric and adult populations. For example, primary tracheal cancers can result in neoplastic lesions, which are often not diagnosed until the tumor has become inoperable. These patients are palliated, but have a poor prognosis, with only $5 \%$ survival after 5 years. Tissue engineered transplants offer a life saving new therapeutic option. Recent reports have demonstrated good midterm results with decellularized human homograft tissue. However, these experiments have been limited to compassionate use. To achieve efficacy necessary for more widespread use further study is necessary to investigate alternate approaches and optimize the decellularization technique. Additionally, clinical application of this technology will require translation to a decellularized xenograft to obviate human tissue supply limitations. To this end, we compare the use of 3 alternate detergents (SDS, Triton X-100, and CHAPS) to sodium deoxycholate in the commonly accepted detergent enzymatic method (DEM). Fresh donor rat tracheas were decellularized using a modified 9-day DEM protocol. The pre-implant scaffolds were thoroughly characterized for each experimental group and implanted for 12 weeks using an orthotopic rat tracheal reconstruction model. It was found that detergent choice strongly affects the host remodeling response including host cell infiltration and epithelial differentiation. The clinically relevant sodium deoxycholate and Triton X-100 groups were retested with a final peracetic acid (PAA) rinse. It was determined that the use of PAA greatly improved the in vivo response of the previously poor performing sodium deoxycholate and made little improvement to the Triton X-100 scaffold. The optimum configuration, Triton X-100 with a PAA rinse, was selected for translation to a clinically relevant porcine model. 
Porcine tracheal decellularization was achieved using a modified 14 day DEM protocol with a novel cyclical pressure approach. The suitability of these porcine tracheas for pre-clinical large animal testing was verified through mechanical analysis (pressure-diameter and suture retention) and in vitro seeding experiments with human bronchial epithelial cells. 


\section{TABLE OF CONTENTS}

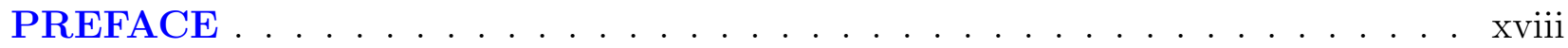

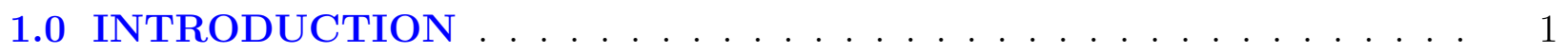

1.1 The Mammalian Trachea . . . . . . . . . . . . . . . . . . . . . 1

1.1.1 Anatomy and Physiology . . . . . . . . . . . . . . . 2

1.1.2 Barrier to Foreign Antigens . . . . . . . . . . . . . . . . 4

1.2 Disease States . . . . . . . . . . . . . . . . . . . 4

1.2 .1 Agenesis \& Atresia . . . . . . . . . . . . . . . . . 4

1.2 .2 Tracheal Stenosis . . . . . . . . . . . . . . . . . 6

1.2 .3 Tracheomalacia . . . . . . . . . . . . . . 6

1.2 .4 Tracheal Tumors . . . . . . . . . . . . . . . . . . . . . 8

1.3 History of Tracheal Surgery . . . . . . . . . . . . . . . . 9

1.3.1 Repair \& Replacement . . . . . . . . . . . . . . . . . . . . 9

1.4 Experimental and Clinical Prostheses . . . . . . . . . . . . . . . . . 11

1.4.1 Synthetic Materials . . . . . . . . . . . . . . . . . . 11

1.4.1.1 Solid Prostheses . . . . . . . . . . . . . . . . . . . . 11

1.4.1.2 Porous Prostheses. . . . . . . . . . . . . . . . . . . . 12

1.4.2 Nonviable Biologic Tissues . . . . . . . . . . . . . . . . . 15

1.4.3 Autograft Transplantation . . . . . . . . . . . . . . 15

1.4.3.1 Free Grafts . . . . . . . . . . . . . . . . . 17

1.4.3.2 Tissue Flaps . . . . . . . . . . . . . . . . . . . . . . 17

1.4.3.3 Tubular Constructs . . . . . . . . . . . . . . . . . . . . 18

1.4.4 Whole Organ Transplantation . . . . . . . . . . . . . . . . . . . 19 
1.4.4.1 Revascularization . . . . . . . . . . . . . . . . . . . . . 19

1.4.4.2 Implant Modification . . . . . . . . . . . . . . 20

1.4 .5 Next Steps . . . . . . . . . . . . . . . . . . . . . 21

1.5 A Living Prosthetic: Tissue Engineered Trachea . . . . . . . . . . . . . . . 22

1.5.1 Synthetic and Biologically Derived Polymers . . . . . . . . . . 23

1.5.2 Decellularized Tissue . . . . . . . . . . . . . . . . . . 23

1.5.3 Cell Lines in Tracheal Tissue Engineering . . . . . . . . . . . . . . . 24

1.5.3.1 Acellular Constructs . . . . . . . . . . . . . . . . 24

1.5.3.2 Single Cell Type . . . . . . . . . . . . . . . 25

1.5.3.3 Multiple Cell Types . . . . . . . . . . . . . . . . . 26

1.5.4 In Vitro Culture . . . . . . . . . . . . . . . . . . . . . . . . . . 27

1.5.5 Surgical Implantation . . . . . . . . . . . . . . . 27

1.5.6 Hurdles to a Tissue Engineered Trachea . . . . . . . . . . . . . 28

1.6 Research Gap \& Specific Approach . . . . . . . . . . . . . . . . . . . . 28

2.0 CHARACTERIZATION OF DECELLULARIZED RAT TRACHEA . 30

2.1 Introduction $\ldots \ldots \ldots \ldots$

2.2 Materials \& Methods . . . . . . . . . . . . . . . . . . . . . . 32

2.2 .1 Animals . . . . . . . . . . . . . . . . . . . . 32

2.2.1.1 Tissue Collection . . . . . . . . . . . . . . . . 33

2.2 .2 Scaffold Preparation . . . . . . . . . . . . . . . . . . . 33

2.2.3 Differential Scanning Calorimetry . . . . . . . . . . . . . 33

2.2 .4 Pressure Diameter Testing . . . . . . . . . . . . . . . . . 35

2.2 .5 Histology . . . . . . . . . . . . . . . . . . . . . . 38

2.2 .6 Statistics . . . . . . . . . . . . . . . . . . . . . 38

2.3 Results and Discussion . . . . . . . . . . . . . . . . . 38

2.3.1 All Treatment Groups Effectively Decellularize Rat Trachea . . . . . . 38

2.3.2 Cartilage Mechanics, Structure and Composition are Maintained . . . 40

2.3.3 Trachea Mucosal Structure is Maintained Despite Cellular Removal . . 40

2.3.4 Detergents Differentially Influence Collagen Microstructure and Matrix Associated Proteins . . . . . . . . . . . . . . . . . 42 
2.3.5 Tracheal Mechanics . . . . . . . . . . . . . . . . . . . . . 49

2.3.6 Limitations and Future Work . . . . . . . . . . . . . . . . . 49

2.4 Conclusions . . . . . . . . . . . . . . . . . . . . . . . 52

2.5 Acknowledgements . . . . . . . . . . . . . . . . . 53

3.0 SUCCESSFUL ORTHOTOPIC TRANSPLANTATION OF DECELLULARIZED RAT TRACHEA . . . . . . . . . . . . . . 54

3.1 Introduction . . . . . . . . . . . . . . . . . . 54

3.2 Materials \& Methods . . . . . . . . . . . . . . . . . . . 55

3.2 .1 Implant . . . . . . . . . . . . . . . . . . . . 55

3.2 .2 Post-operative Care . . . . . . . . . . . . . . . . . . . 55

3.2.3 Explant and Tissue Processing . . . . . . . . . . . . . 57

3.2 .4 Ciliary Beat Frequency . . . . . . . . . . . . . . . . 57

3.2 .5 Scanning Electron Microscopy _. . . . . . . . . . . . . 58

3.2 .6 Histology . . . . . . . . . . . . . . . . . . . . . 58

3.2 .7 Statistics . . . . . . . . . . . . . . . . . . . 58

3.3 Results and Discussion . . . . . . . . . . . . . . . . . 60

3.3.1 Animal Survival Varies with Treatment, PAA Correlated with Positive

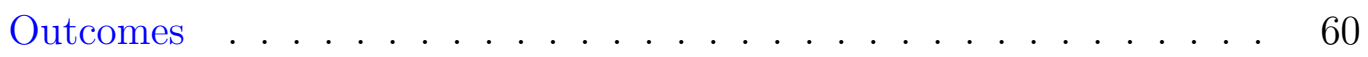

3.3.2 Mortality Driven by Obstruction . . . . . . . . . . . . . . 61

3.3.3 Processing Conditions Predetermines Host Mediated Remodeling . . . 62

3.3.4 Decellularized Grafts Support Epithelial Growth and Differentiation . 67

3.3.5 PAA treated Grafts Regenerate a Functional Immunological Barrier 69

3.3 .6 Limitations and Future Work . . . . . . . . . . . . . . . . 74

3.4 Conclusions . . . . . . . . . . . . . . . . . . . . . . . . . 74

3.5 Acknowledgements . . . . . . . . . . . . . . . . . . 77

4.0 TRANSLATING TO A CLINCIALLY RELEVANT XENOGENEIC

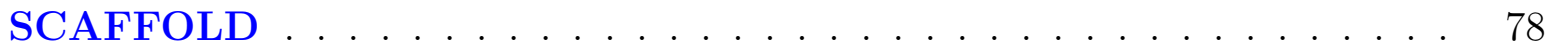

4.1 Introduction $\ldots \ldots \ldots \ldots \ldots \ldots$

4.1.1 Cyclical Pressure Decellularization . . . . . . . . . . . . . . 79 
4.1.1.1 Pressured Assisted Decellularization Improves Retention of Native Mechanical Properties . . . . . . . . . . . . . . 80

4.1.1.2 Demonstrated Success of Cyclical Pressure Technique in Three Month Large Animal Implants . . . . . . . . . . . . . . . 85

4.2 Materials \& Methods . . . . . . . . . . . . . . . . . . . . . . . . . 88

4.2 .1 Tissue Collection . . . . . . . . . . . . . . . . . . 88

4.2.2 Porcine Trachea Vacuum Decellularization . . . . . . . . . . . . 91

4.2 .3 Suture Retention Testing . . . . . . . . . . . . . . . . . . . . . 92

4.2.4 Pressure Diameter Testing . . . . . . . . . . . . . . . . . . . . 92

4.2 .5 In vitro Cell Culture . . . . . . . . . . . . . . . . . . . . 92

4.2 .6 Histology . . . . . . . . . . . . . . . . . . . . . . . . . 93

4.2 .7 Statistics . . . . . . . . . . . . . . . . . . . 94

4.3 Results and Discussion . . . . . . . . . . . . . . . . . . . 94

4.3.1 Cyclical Vacuum Capable of Successful Porcine Tracheal Decellularization 94

4.3.2 Xenogeneic Scaffold is Mechanically Suitable for Transplant . . . . . . 94

4.3.3 Porcine Scaffolds Support Healthy Human Respiratory Epithelium . 97

4.3.4 Limitations and Future Work . . . . . . . . . . . . . . . . . . 97

4.4 Conclusions . . . . . . . . . . . . . . . . . . . . . . . . . 97

4.5 Acknowledgments . . . . . . . . . . . . . . . . . . . . . 99

5.0 SUMMARY AND CONCLUSIONS . . . . . . . . . . . . . . 100

5.1 Novel Findings and Future Directions _ . . . . . . . . . . . . . . 100

5.1.1 Structural and Compositional Characterization of Decellularized Scaffolds is Vital to Tissue Engineering Success . . . . . . . . . . . . 101

5.1.2 Processing Conditions Predetermines Host Mediated Remodeling . . . 103

5.1.3 Peracetic Acid (PAA) Treated Grafts Regenerate a Functional Immunological Barrier . . . . . . . . . . . . . . . . 105

5.1.4 Cyclical Pressure Synergistically Improves Detergent Based Decellularization . . . . . . . . . . . . . . . . . . 108

5.1.5 Xenogeneic Scaffolds are Suitable for Further Preclinical Testing . . . 109

5.2 Closing Remarks . . . . . . . . . . . . . . . . . . . . . . . 111 
BIBLIOGRAPHY . . . . . . . . . . . . . . . . . . . 112 


\section{LIST OF TABLES}

1.1 Solid Prostheses . . . . . . . . . . . . . . . . . . . . . . . . . . . . . . . 13

1.2 Porous Prostheses . . . . . . . . . . . . . . . . . . . . . . . . . 14

1.3 Outcomes for Nonviable Biological Prostheses . . . . . . . . . . . . . . 16

1.4 Requirements for a Suitable Tracheal Replacement . . . . . . . . . . . . . 21 


\section{LIST OF FIGURES}

1.1 Illustration of a Human Trachea . . . . . . . . . . . . . . . . . . 1

1.2 Larynx and proximal trachea, reproduced from Gray's Anatomy . . . . . . . 3

1.3 Schematic of a transverse tracheal section, reproduced from Gray's Anatomy 3

1.4 Scanning electron microscope image of lung trachea epithelium. There are both ciliated and non-ciliated cells in this epithelium. Note the difference in size between the cilia and the microvilli (on the non-ciliated cell surface). Adapted from "Bronchiolar epithelium 3 - SEM" by Charles Daghlian . . . . 5

1.5 "Laryngotracheal Stenosis" by Rn Cantab licensed under CC 3.0 Unported . 7

1.6 Julius Casserius - Tracheotomy Tabulae Anatomicae 1601 . . . . . . . . . . . 10

2.1 Decellularization schematic derived from the clinically accepted detergent enzymatic method. Each day specimens were subjected to two hours in the specified detergent and three hours in 1M sodium chloride with 2,000 KU DNAse I with gentle agitation at $20^{\circ} \mathrm{C}$. Scaffolds were rinsed and stored overnight in PBS with $1 \%$ antibiotic/antimycotic. This process was repeated for nine days. Scaffolds were then individually packaged and subjected to terminal gamma ray sterilization . . . . . . . . . . . . . . . .

2.2 A custom built device designed to simultaneously test the pressure and diameter response of tracheas under physiologically relevant levels of air loading.

2.3 Screenshot from custom matlab code. A: Automated cycle identification and data smoothing, excluded data is shown in red. B: Extraction of average pressure and diameter curves. . . . . . . . . . . . . . . . . 
2.4 Gross morphology of rat tracheas after DEM decellularization prior to gamma sterilization. Decellularization preserved organ level structure and shape. Translucent appearance suggests successful decellularization. . . . . . . . . .

2.5 10X Alcian blue stained detergent decellularized trachea scaffolds. Glycoaminoglycans are stained blue with nuclear material in purplish red. All grafts demonstrate maintenance of GAGs within intact cartilage rings. As seen in the CHAPS, SDS, Triton, and deoxycholate groups, nuclei and nuclear material are absent in all decellularized specimens . . . . . . . . . . . . . .

2.6 10X Alcian blue stained PAA treated decellularized trachea. Grafts demonstrate retention of GAG within intact cartilage rings. . . . . . . . . . . .

2.7 10X Movat's pentachrome staining of non PAA treated, decellularized rat tracheas. Black: nuclei and elastic fibers, Yellow: collagen, Blue: mucin, Bright Red: fibrin, Red: Muscle. Partial removal or denaturation of basement membrane and elastic fibers is evident within ionic detergent groups (SDS and

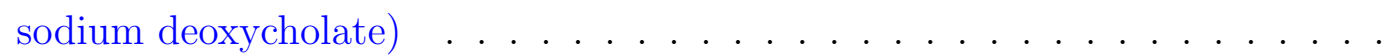

2.8 10X Movat's pentachrome staining of PAA treated, decellularized rat tracheas. Black: nuclei and elastic fibers, Yellow: collagen, Blue: mucin, Bright Red: fibrin, Red: Muscle. . . . . . . . . . . . . . . . . .

2.9 Differential scanning thermograph of non PAA treated decellularized rat tracheas. Primary collagen peak is located at $60^{\circ} \mathrm{C}$, with mature highly crosslinked fibers stable up to 75 or $80^{\circ} \mathrm{C}$. Matrix associated proteins including elastin and GAGs are represented as a broad shoulder from $35^{\circ} \mathrm{C}$. . . . .

2.10 Differential scanning thermograph demonstrating comparison between PAA treated and untreated detergent decellularized rat tracheas. . . . . . . . . .

2.11 10X Herovivi's staining of non PAA treated, decellularized rat tracheas, primarily esed to differentiate young and mature collagen. Herovici's stains young collagen and reticulum blue and mature collagen red while providing a yellow cytoplasm counterstain. Nuclei are stained blue to black. . . . . . . 
2.12 Main collagen peak and shoulder onset temperature computed from DSC thermogram, presented as mean plus/minus standard deviation (minimum $\mathrm{n}=3$ for each group). Top: Main peak temperature representing the maximal value for the largest peak, computed as the global minimum of the zero first derivative, SDS did not have a clear peak and was therefore, excluded. Bottom: Shoulder onset temperature for the main peak. Computed as the temperature at which a 5 degree moving average first deviated from a linear baseline. . . . . . . . . . . . . . . . . . . .

2.13 Total enthalpy, computed from DSC thermogram, presented as mean plus/minus standard deviation (minimum $\mathrm{n}=3$ for all groups). Computed as the total area between the curve and a fitted linear baseline. Statistical significance was calculated with a MANOVA and post hoc Tukey's test, significant differences were found between all detergent only specimens and both PAA treated groups. A significant difference was also found between SDS and the remaining detergent only specimens. . . . . . . . . . . . . . . . . .

3.1 Intraoperative images of orthoptopic transplantion. Top: Decellularized trachea after graft reanastomosis prior to strap muscle reapproximation and closure. Bottom: Zoomed image demonstrating careful separation of the neurovascular bundle and graft alignment . . . . . . . . . . . . . .

3.2 Schematic of ciliary beat frequency analysis. Top: The freshly explanted and transected trachea is sandwiched between a glass coverslip and glass culture well, silicone sheeting is used as a spacer. Middle: DIC microscopy is used to capture at least 400 frames of cilia motion. The video is imported into ImageJ and a line is manually drawn through the beating cilia. Bottom: The video is re-sliced along the line by time, resulting in a plot with pixel intensity plotted along the $\mathrm{x}$ axis for each video frame. Ciliary beat frequency is computed manually by measuring cyclic pixel variation over time . . . . . . . . . .

3.3 Post-operative survival curves demonstrating increased survival among SDS and Triton X-100 experimental groups. 
3.4 Post-operative survival curves showing 100\% survival among both PAA posttreated experimental groups. . . . . . . . . . . . . . . . .

3.5 Top: Gross appearance of CHAPS graft at necropsy. Middle CHAPS necropsy H\&E section demonstrating mucous obstruction in red, Bottom: Triton necropsy also demonstrating lack of stenosis and muscous obstruction in red. . . . . .

3.6 Gross morphology of representative explanted tracheal grafts. Significant stenosis in CHAPS and sodium deoxycholate groups. All non-PAA treated grafts demonstrated a yellow hue, potentially suggesting the onset of necrosis. Neovasculature is not readily apparent in the CHAPS, SDS, or Triton X-100 groups. Treatment with PAA dramatically improves graft appearance at explant. PAA treated grafts demonstrate a healthy, native appearance with distinct vasculature . . . . . . . . . . . . . . . . . .

3.7 Left: Scanning electron micrograph (1000X) of the native rat trachea. Right: Expanded view $(5000 \mathrm{X})$ of dense mature ciliated epithelium . . . . . . . 68

3.8 Representative scanning electron micrograph (25X) of 12 week CHAPS explant. Right frame shows expanded view (500X) of squamous epithelium . .

3.9 Representative scanning electron micrograph of 12 week SDS explant. Left: 100X magnification Right frame shows expanded view (1,000X) of squamous epithelium with several ciliated cells in the bottom right corner . . . . . . .

3.10 Representative scanning electron micrographs of 12 week Triton X-100 explants with expanded views. Top: Non-PAA treated control showing a patent lumen with good epithelial recolonization and differentiation. Bottom: PAA treated Triton X-100 graft showing excellent epithelial coverage with mature cilia. . . . . . . . . . . . . . . . . . . . .

3.11 Representative scanning electron micrographs of 12 week sodium deoxycholate explants with expanded views. Top: Non-PAA treated control showing squamous epithelium with distributed patches of bare ECM (100X) . Bottom: PAA treated Triton X-100 graft showing excellent epithelial coverage with mature cilia. Right frame shows expanded view of squamous epithelium . . 
3.12 Ciliary beat frequencies computed from 12 week non PAA treated Triton X100 explants $(\mathrm{n}=5)$. Explanted tracheal sections, both graft and native, have cilia function significantly lower than naive controls. . . . . . . . . . . . 72

3.13 Ciliary beat frequencies computed from 12 week PAA treated Triton X-100 and sodium deoxycholate explants $(\mathrm{n}=5$ each). Naive cilia function is restored among all PAA treated grafts . . . . . . . . . . . . . . .

3.14 Histologic examination of basal cell protein expression within decellularized mouse tracheal grafts. Tracheas were explanted at 1, 4, and 8 weeks following surgery and were examined for expression of keratins 5 (K5, red) and 14 (K14, green), followed by DAPI staining (blue). Dual-positive cells are highlighted in yellow. Scale bar 200 microns. . . . . . . . . . . . . . . . . . . . . . 75

3.15 Explants of mice one, four, or eight weeks following surgery and immunofluorescent labeling for markers of mature epithelial cells was performed, followed by DAPI-staining (blue). Images are shown at a mid-graft location. Cells expressing acetylated tubulin (ACT, red) and Clara cell secretory protein (CCSP, green) are shown . . . . . . . . . . . . . . .

4.1 Cyclical pressure device comprised of a PC controlled vacuum pump, solenoid valves, pressure sensor, and vacuum chamber. Pressure is cyclically pulsed with tissue submerged in a detergent to effect decellularization . . . . . . . .

4.2 H\&E sections of: Native porcine dermis (Top) and Cyclical Pressure Decellularization $($ Bottom $) \ldots \ldots \ldots \ldots$

4.3 Plots comparing maximum stiffness and failure stress for cyclical pressure decellularized, agitation controls, and native vocal folds. Please note the significant difference between agitation controls and both cyclical pressure decellularized and native tissue for both measured parameters. . . . . . . .

4.4 Viscoelastic properties of cyclical pressure decellularized, agitated controls, and native vocal folds. Agitated controls were found to be significantly different than both cyclical pressure decellularized specimens and the native control. No significant difference was observed between native and cyclical pressure decellularized specimens. . . . . . . . . . . . . . . . 
4.5 Comparison of hematoxylin and eosin staining for native, agitation control, and cyclical pressure decellularized porcine aorta demonstrating improved retention of tissue micro-structure in pressure assisted techniques compared to standard agitation decellularization. . . . . . . . . . . . .

4.6 Surgical images demonstrating the graft prior to implant (top) and completed surgery immediately before closure (bottom) . . . . . . . . . . . . . 89

4.712 week explanted cyclical pressure decellularized specimens . . . . . . . . . 90

4.8 Chart demonstrating programmed pressure profile for three decell cycles taking approximately one minute each . . . . . . . . . . . . . . . . . 91

4.9 Hemotoxylin \& Eosin staining of native (top) and vacuum decellularized (bottom) porcine trachea . . . . . . . . . . . . . . . . .

4.10 Left: Suture retention curves showing load elongation. Mean and standard deviation for each group is presented. Right: Suture retention setup showing a reanastomosed trachea clamped prior to uniaxial load to failure testing on

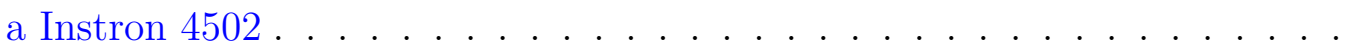

4.11 Histology of fixed tracheal mucosa after 72 hours seeding in vitro with human bronchial epithelial cells. Top: Differential interference microscopy stack showing confluent epithelium. Bottom: FITC conjugated phalloidin stained epithelial cells . . . . . . . . . . . . . . . . . . . 


\section{PREFACE}

To my grandfather, I wish he'd been around to see this. 


\subsection{INTRODUCTION}

\subsection{THE MAMMALIAN TRACHEA}

The trachea or windpipe is a cartilaginous and membranous tube, extending from the lower part of the larynx, on a level with the sixth cervical vertebra, to the upper border of the fifth thoracic vertebra, where it divides into two bronchi (Figure 1.1). The trachea is a posteriorly flattened cylinder, measuring about $12 \mathrm{~cm}$ in length and approximately $2 \mathrm{~cm}$ in diameter. In children the trachea is approximately $6 \mathrm{~cm}$ in length and $1 \mathrm{~cm}$ in diameter.

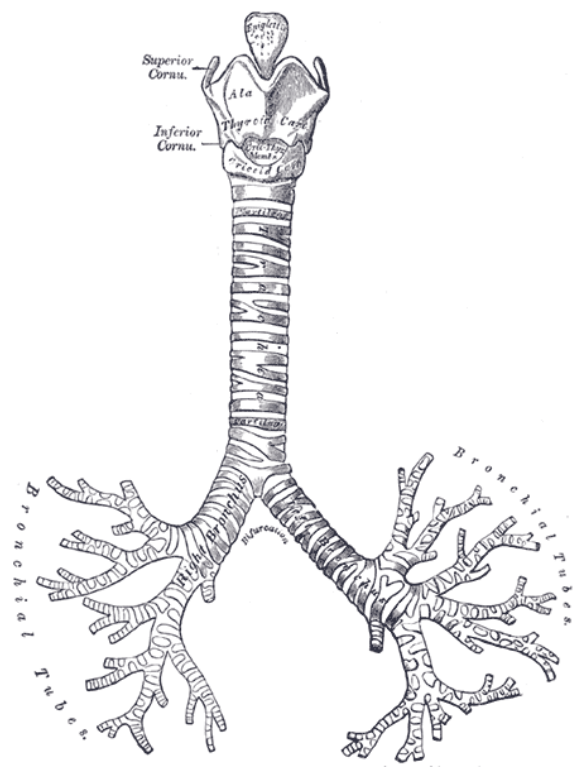

Figure 1.1: Human Trachea, reproduced from Gray's Anatomy 


\subsubsection{Anatomy and Physiology}

The trachea is composed of partial circumference rings of hyaline cartilage, fibrous tissue, muscular fibers, and a mucous membrane. There are typically between sixteen and twenty cartilage rings occupying the front two-thirds of the trachea. A simple membrane composed of fibrous tissue muscular fibers completes the c-shaped cartilage rings. The cartilage rings are horizontally above one another, separated by narrow fibrous regions, the annular ligaments (Figure 1.1). Tracheal rings measure about $4 \mathrm{~mm}$ in height and $1 \mathrm{~mm}$ in thickness. Often, two or more of the cartilages combine, partially or completely, and may be bifurcated at their extremities. The first and last tracheal rings are unique, the first is broader and connected by the cricotracheal ligament to the bottom of the cricoid cartilage (Figure 1.2). The last cartilage is thick and broad curving downward between the two bronchi. The cartilage rings are enclosed in an elastic fibrous membrane, which consists of two layers; the thicker passes over the outer surface of the ring, the thinner over the inner surface. On either side of the cartilage the two layers blend together to form a single membrane, which connects the rings to one another. The mucous membrane consists of loose connective tissue, with a distinct basement membrane, supporting a stratified epithelium. The surface layer is comprised of a variety of specialized epithelial cell types, including sensory, secretory, and ciliated epithelium. The deeper layers are composed of cuboidal basal cells thought to have some ability to differentiate into other cells types found within the epithelium. Basal cells can respond to injury of the airway epithelium, migrating to cover a site denuded of epithelial cells, and subsequently differentiating to restore a healthy epithelial cell layer. Beneath the basement membrane there is a distinct layer of longitudinal elastic fibers with a small amount of intervening connective tissue. The submucous layer is composed of a loose mesh work of connective tissue, containing large blood vessels, nerves, and mucous glands with ducts opening to the surface (Figure 1.3). The trachea is finely tuned to its intended function. The combination of C-Shaped cartilage rings and the fibrous annular ligaments makes it circumferentially stiff, but longitudinally compliant. This mechanical anisotropy enables relative mobility of the head, but also prevents collapse during respiration. In addition to its mechanical role, the trachea has a vital immunologic role. 


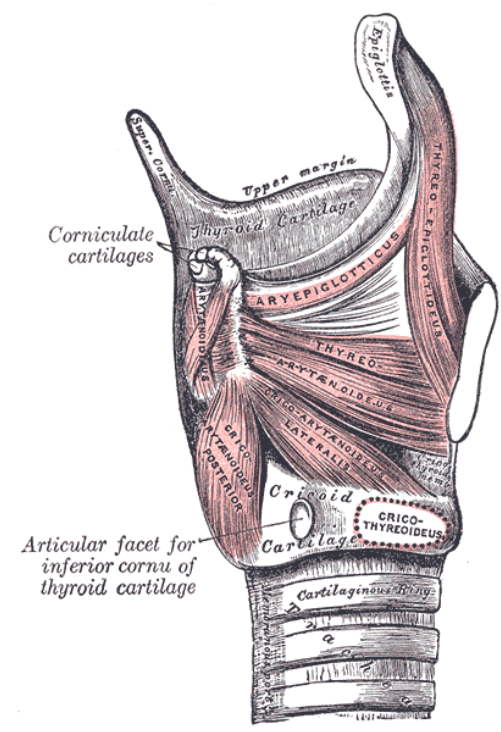

Figure 1.2: Larynx and proximal trachea, reproduced from Gray's Anatomy

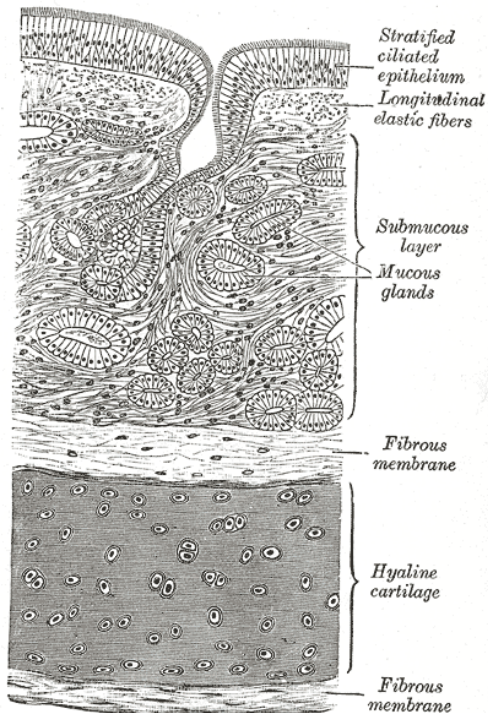

Figure 1.3: Schematic of a transverse tracheal section, reproduced from Gray's Anatomy 


\subsubsection{Barrier to Foreign Antigens}

The trachea possesses many exocrine glands and is lined by a pseudo-stratified columnar epithelium in which ciliated and mucous cells predominate. The ciliated epithelial cells are interspersed with goblet mucousal cells (named for the similarity in shape to a wine glass). Goblet cells produce mucous which helps maintain epithelial moisture and traps particulate matter and pathogens moving through the airway. The ciliated cells then beat in concert, moving secreted mucus containing trapped foreign particles toward the laryngopharynx, for either expectoration or swallowing to the stomach where the acidic pH helps to neutralize microorganisms (Figure 1.4). This system is collectively known as the mucociliary escalator and serves two functions: to keep the lower respiratory tract sterile, and to prevent mucus accumulation in the lungs.

\subsection{DISEASE STATES}

Due to the complexity of the human respiratory system, there are many conditions effecting the larynx, bronchi, lungs and trachea. However, this overview will focus solely on those conditions affecting the trachea. While rare, conditions affecting the trachea are particularly troubling to surgeons. The following sections will present an overview of the important conditions and their clinical presentation.

\subsubsection{Agenesis \& Atresia}

In children, tracheal agenesis and atresia are almost uniformly fatal and are fortunately quite rare. The trachea may be completely absent (agenesis), or it may be partially in place but considerably underdeveloped (atresia). Operative techniques are available to correct the underlying abnormality, but surgical attempts have yielded poor results, essentially making this an uncorrectable malformation. 


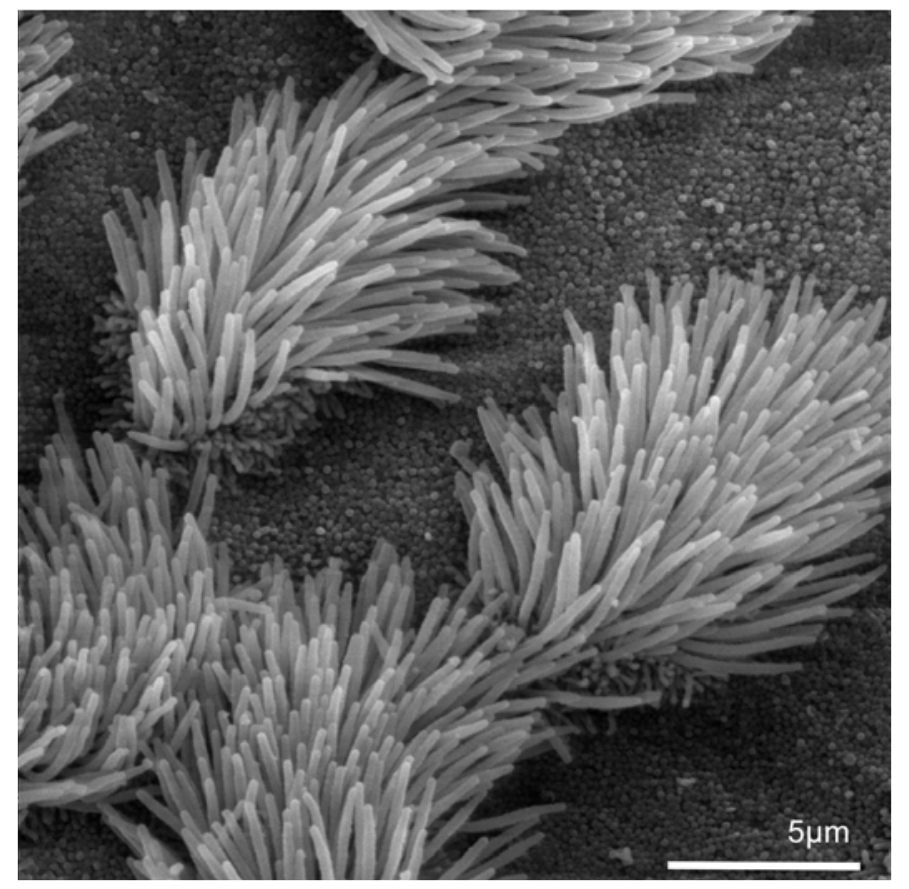

Figure 1.4: Scanning electron microscope image of lung trachea epithelium. There are both ciliated and non-ciliated cells in this epithelium. Note the difference in size between the cilia and the microvilli (on the non-ciliated cell surface). Adapted from "Bronchiolar epithelium 3 - SEM" by Charles Daghlian 


\subsubsection{Tracheal Stenosis}

Tracheal stenosis refers to an abnormal narrowing of trachea below the vocal folds, as opposed to those present in the main bronchi or larynx. This is often referred to as subglottic stenosis in children as this is the narrowest region of the trachea and where stenosis is most likely to present. Stenosis encompasses a wide variety of conditions, including both acquired and congenital defects. The most common cause for stenosis is post-intubation and tracheotomy related injuries. Stenosis may involve a single cartilage ring, or the entire length of the trachea (Figure 1.5) Typically all cases of stenosis regardless of cause result in a similar clinical presentation, including breathlessness (dyspnea) particularly when undertaking physical activities (exertional dyspnea). The patient may also experience added respiratory sounds which in the more severe cases can be identified as stridor (a high-pitched sound resulting from turbulent air flow in the upper airway) but in many cases can be readily mistaken for lower respiratory distress. Thus, many patients with tracheal stenosis are incorrectly diagnosed and treated as asthmatics. In children these conditions are often not discovered until a secondary cause such as an upper respiratory infection increasingly taxes the already limited airway.

\subsubsection{Tracheomalacia}

Tracheomalacia is a condition in which the cartilage rings are not sufficiently stiff to support the trachea from pressures generated by respiration. During normal function, the trachea dilates slightly during inspiration and narrows during expiration. In a malacic trachea,

the membranous portion of the trachea compresses inwards markedly reducing the airway lumen. In severe cases this can result in total collapse of the airway during expiration. Primary tracheomalacia is a congenital disorder of the tracheal rings and is relatively rare. On the other hand, secondary tracheomalacia is an acquired disorder in which cartilage weakness results from an external pressure. Common causes of secondary tracheomalacia include vascular anomalies, such as innominate artery compression and tracheoesophageal fistula (abnormal connections between the esophagus and trachea). Clinical presentation typically involves breathing problems that worsen with coughing, crying, feeding, or upper 


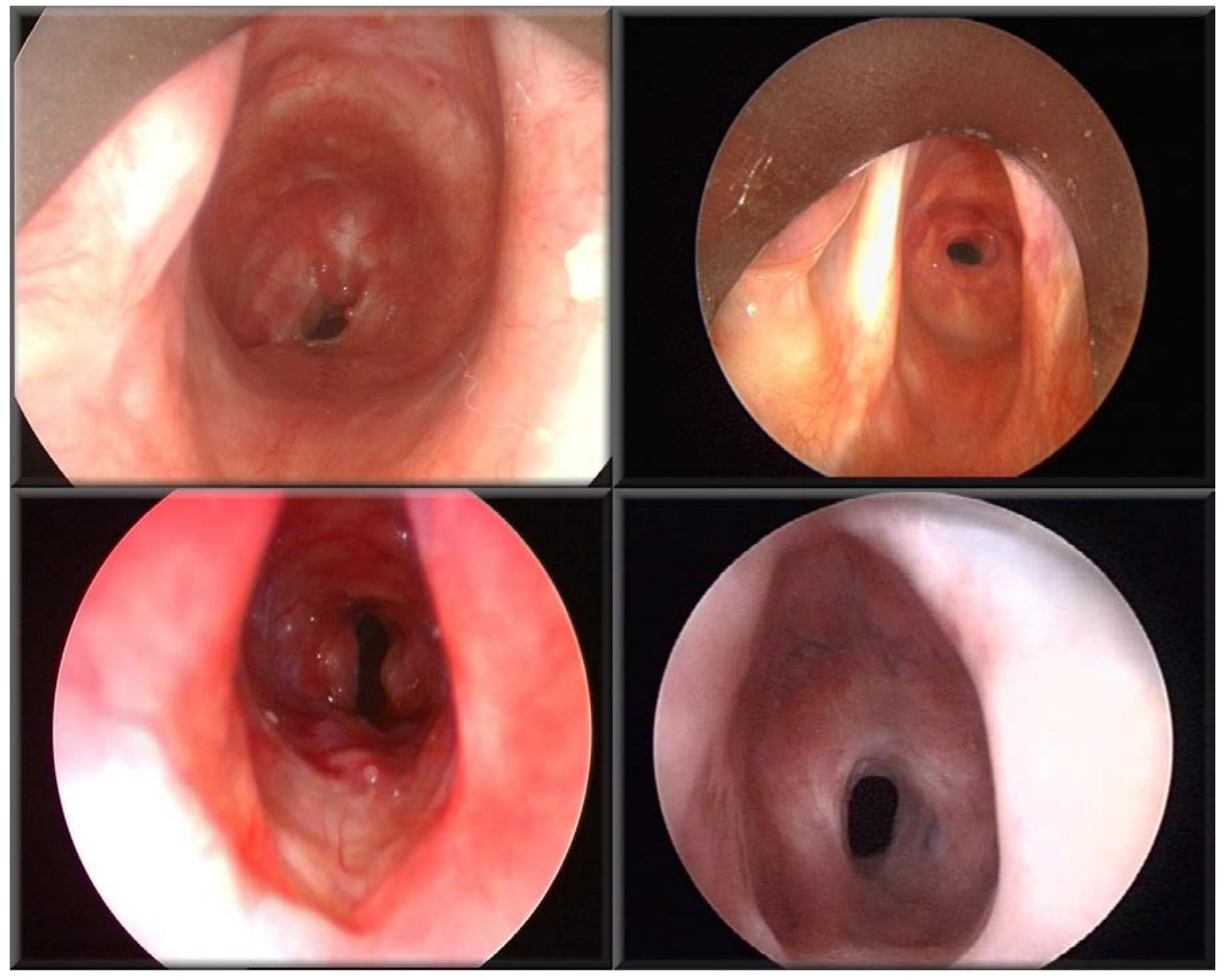

Figure 1.5: "Laryngotracheal Stenosis" by Rn Cantab licensed under CC 3.0 Unported 
respiratory infections, in severe cases, infants may hyper-extend their necks in order to keep the airway open.

\subsubsection{Tracheal Tumors}

Trachea tumors whether benign or malignant can affect respiration through obstruction of the airway. Malignant primary tracheal tumors are most often smoking related squamous cell carcinomas (SCC). One quarter of tumors are benign: chondromas from the cartilage rings, hemangiomas, result from tumors in tiny blood vessels, and papillomas are human papilloma (HPV) viral tumors. In addition to primary neoplasms, secondary involvement can occur from tumors of surrounding tissues such as the lungs, larynx, and esophagus. Typically, tracheal tumors grow slowly. Malignant tumors, specifically squamous cell carcinomas, may be ulcerative. Primary tracheal tumors are insidious, often presenting with signs and symptoms of upper airway obstruction. While readily treatable at early stages, they are often not diagnosed until 6 months after first symptoms present. In a series of 329 patients with primary tracheal malignancies, dyspnea was the most frequent symptom (71\%), followed by cough (40\% with blood (34\%), asthma (19.5\%), and stridor (17.5\%) [1]. The first symptom is usually exertional dyspnea, which gradually worsens. Acute respiratory difficulty is usually not present until the airway is nearly fully occluded. Delay in diagnosis occurs since chest radiograms are the standard diagnostic technique. Patients may also present with repeated episodes of either unilateral or bilateral pneumonia. A diagnosis of adult-onset asthma is often made, delaying definitive treatment. In one series, delayed diagnosis of more than 6 months after symptoms onset occurred in one third of patients. SCC metastasizes to regional lymph nodes and can invade the mediastinum in more aggressive forms or in late stages. Distant metastases are common, most often to bone. Adenoid cystic carcinoma (ACC) occurs almost as frequently as SCC, collectively comprising two thirds

of primary tracheal malignancy. It is slower in onset than SCC, often having a prolonged course of clinical symptoms. 


\subsection{HISTORY OF TRACHEAL SURGERY}

The tracheotomy is arguably the oldest surgical procedure described, evidence as far back as hieroglyph slabs belonging to King Djer in Abydos and King Aha in Saqqara suggest the tracheotomy was performed in ancient Egypt around 3100 BC. The tracheotomy is also mentioned in the Rig Veda, a sacred Hindu medical text written before 1000BC [2]. In the fourth century, Alexander the Great is said to have saved a soldier choking on a bone by puncturing his trachea with the point of his sword. Scant mention was made of tracheal surgery again until the 16th century, it was considered irresponsible and dangerous due to risk of infection and perceived inability of cartilage to heal. Successful tracheotomies were only recorded 28 times from 1546 until 1833. In these cases it was used only in emergency cases of laryngeal abscess, severe respiratory distress, or obstructive blockage (Figure 1.6). In one report a tracheotomy was used to relieve pressure caused by impingement from gold coins lodged in the esophagus of a 14-year-old boy who had swallowed them to prevent their theft [3]. Over time, the tracheotomy became an accepted technique to bypass upper respiratory obstruction caused by infection or foreign bodies. However, further work remained to refine the tools and techniques. High incision, improper cannulas, splitting the cricoid cartilage predisposed patients to complications. The use of a curved cannula removing pressure from the anterior and posterior surfaces of the trachea prevented lesions and erosion. These advances significantly decreased mortality and morbidity. Recently it has become clear that endotracheal intubation is quicker and safer, mostly relegating the use of tracheotomy for patients requiring long term mechanical ventilation.

\subsubsection{Repair \& Replacement}

Lingering ancient fears were a major obstacle to the development of tracheal surgical procedures. Up until the late 20th century it was widely believed that the limited inherent healing capacity of cartilage presented an insurmountable challenge. Hippocrates argued that "The most difficult fistulas are those which occur in the cartilaginous areas...". In the second century AD Aretaeus pronounced "The lips of the wound do not coalesce, for they are 


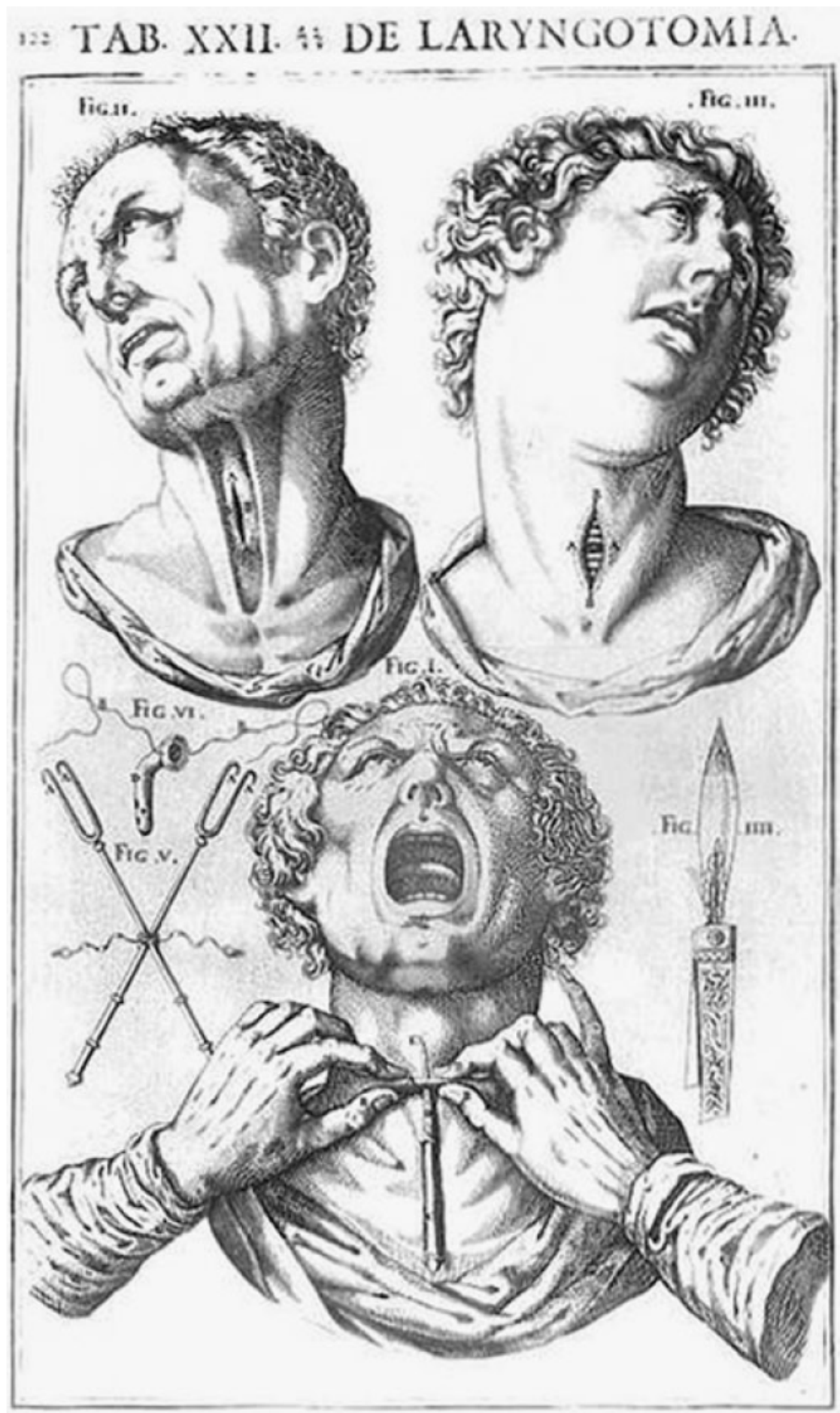

Figure 1.6: Julius Casserius - Tracheotomy Tabulae Anatomicae 1601 
both cartilaginous and not of the nature to unite" [3]. Another significant concern was the long held belief that only a limited segment of trachea could be removed and re-anastomosis accomplished. Belsey, among many others, believed that roughly $2 \mathrm{~cm}$ of the human trachea could be excised and the trachea re-anastomosed [4]. These various reports sparked a nearly century long search for a safe and efficacious replacement.

\subsection{EXPERIMENTAL AND CLINICAL PROSTHESES}

In general, research has been conducted among several parallel avenues

1. Synthetic materials, both solid and porous to encourage host infiltration

2. Nonviable biological tissues, including fixed trachea

3. Autograft transplantation

4. Allograft transplantation

Success has been announced periodically, but so far no single replacement has been safe and efficacious in the long term.

\subsubsection{Synthetic Materials}

The apparent simplicity of a tracheal replacement encouraged trials of tubular constructs throughout the 20th century. These synthetic materials were predominantly simple tubes, fabricated from inert, biocompatible materials. Problems of migration, dislodgement, infection, and obstruction inevitably arose. Furthermore, these tubes became permanent implants, since connective tissue eventually forms around the grafts, causing obstruction and stenosis in the absence of a stent [5].

1.4.1.1 Solid Prostheses Synthetic materials were used extensively to form solid, tubular conduits as replacements for resected trachea. Most often, experimental designs were tested in dogs. Materials employed include, stainless steel, plastics, cobalt alloys, silicone rubbers, methacrylates and are listed exhaustively in Table 1.1. Some of these designs were 
even attempted clinically. For example, Borrie and colleagues [6, 7] added fabric cuffs to silicone tubes to hopefully prevent granulation tissue and to encourage fixation of the prosthesis. Neville et al used a similarly cuffed silicone tube clinically [8]. Toomes and Grillo both experienced obstructive granulation tissue, migration, and vascular erosion with the same prosthesis $[9,10]$. Success was variable, but all grafts eventually tended to migrate, become dislodged, obstructed and encourage infection at the graft interface. Erosion of the brachiocephalic artery was periodically present and often fatal. Despite all of these complications, a rigid prosthesis can maintain an open airway temporarily, even in the absence of a healing response. Further attempts were made with porous materials to encourage tissue ingrowth, reepithelization, and prevent dislodgement.

1.4.1.2 Porous Prostheses Meshes fabricated from a wide variety of materials were employed both experimentally and clinically. Host cell infiltration and deposition of connective tissue was hoped to better support epithelial migration. Fabric meshes were typically supported with wire, plastic rings, or coils and wrapped with with biopolymers such as fibrin or collagen to ensure an air tight seal at implant. Meshes tested experimentally, include steel, stainless, titanium, polyethylene, Teflon, polyurethane, Dacron (polyester) and Polyvinyl acetal, but are listed exhaustively in Table 1.2. Meshes were also used clinically, with attempts using steel wire, tantalum covered with fascia or skin, HDPE sometimes covered with pericardium; and polyvinyl acetal and steel wire. The deposition of scar tissue in these grafts often led to obstruction and stenosis. Frequently, large sections of mesh remained bare, resulting in bacterial colonization. Only those grafts that were sufficiently short were fully recellularized. As with many solid prostheses, arterial erosion was present with HDPE replacements in the clinic $[30,31]$. Death occurred with steel mesh prosthesis [13]. HDPE mesh that fails to become fully epithelialized is a haven for environmental bacteria, especially Pseudomonas aeruginosa, which produces a horrific odor. One patient, over many years had lost his wife, his job, and friends because of intolerable halitosis. He was finally relieved by the replacement of his HDPE graft with with a silicone T tube [10]. 
Table 1.1: Solid Prostheses

\begin{tabular}{|c|c|c|}
\hline \multicolumn{3}{|l|}{ Animal Experimentation } \\
\hline Material & Year & Publication \\
\hline Stainless Steel & $1948 \& 1973$ & {$[11,12]$} \\
\hline Steel Coil & 1956 & {$[13]$} \\
\hline Cobalt Chromium Alloy & $1948 \& 1961$ & {$[11,14]$} \\
\hline Glass & 1948 & {$[11]$} \\
\hline Polyethylene & $1948,1949,1953,1955 \& 1968$ & {$[5,15,16,17,18]$} \\
\hline PMMA & $1956 \& 1961$ & {$[13,14]$} \\
\hline Silicone Rubber & $1969,1970, \& 1973$ & {$[6,7,19]$} \\
\hline Teflon(PTFE) & $1963,1970 \& 1973$ & {$[12,20,21]$} \\
\hline Polyvinylacetal & $1958 \& 1961$ & {$[14,22]$} \\
\hline Polyvinylchloride & 1958 & {$[23]$} \\
\hline \multicolumn{3}{|l|}{ Human Implantation } \\
\hline Stainless Steel & $1950 \& 1952$ & {$[24,25]$} \\
\hline Steel Coil & 1956 & [13] \\
\hline Silicone Rubber & $1985 \& 1990$ & {$[9,8]$} \\
\hline Polyethylene & $1952,1962 \& 1965$ & {$[26,27,28]$} \\
\hline Teflon(PTFE) & 1959 & {$[29]$} \\
\hline PMMA & 1948 & {$[15]$} \\
\hline
\end{tabular}


Table 1.2: Porous Prostheses

\begin{tabular}{llr}
\hline Animal Experimentation & & \\
\hline Material & Year & Publication \\
Steel Wire & $1951-1970$ & {$[5,13,18,21,32,33,34]$} \\
Tantalum & $1949-1968$ & {$[35,36,13,18,21,37,38,39]$} \\
Titanium & 1984 & {$[40]$} \\
High Density Polyethylene (HDPE) & $1962 \& 1968$ & {$[18,33,41,30]$} \\
HDPE \& Collagen & 2000 & {$[42]$} \\
Collagen \& Polypropylene & $1994 \& 1997$ & {$[21,45,46,28]$} \\
Teflon & $1965-1990$ & {$[47]$} \\
Polyurethane & 1983 & {$[14,48]$} \\
Polyvinyl acetal & $1958 \& 1961$ & {$[49]$} \\
Polyester (Dacron) & 1988 & {$[13,32]$} \\
\hline Human Implantation & & {$[50,51]$} \\
\hline Steel Wire & $1951-1956$ & {$[30,52,31]$} \\
Tantalum with skin or fascia & 1952 & {$[47]$} \\
HDPE with pericardium & $1965,1968 \& 1975$ & \\
Polyvinyl acetal \& steel wire & 1983 & {$[25$} \\
\hline
\end{tabular}




\subsubsection{Nonviable Biologic Tissues}

Given the frustrating results obtained experimentally and clinically from synthetic materials, researchers naturally attempted alternative material sources. A series of studies were conducted employing cadaver trachea and other tissues, chemically fixed, cryopreserved, or lyophilized. Scherer et al attempted gluteraldehyde fixed rat and pig trachea. Segments were implanted as allografts and autografts. As expected, rejection was not observed, but gross morphological survival of cartilage was only observed in rats [53]. Sterilized and lyophilized aortic allografts over polyethylene tubes were implanted in dogs, while the stents prevented stenosis, longitudinal contraction damaged the proximal and distal ends of the trachea [54]. Marrangoni and Greenberg observed that lyophilized canine tracheal allografts lost their cartilage and were replaced by scar tissue $[55,56]$. Bjork and Rodriguez implanted lyophilized, formalin fixed and ethanol treated allografts. These grafts were uniformly fatal [22]. In general, nonviable biological tissues are eventually degraded and replaced over time by scar tissue. Clinically, human tracheal grafts fixed in formalin and stored in merthiolate were implanted and observed for more than 13 months. These scaffolds demonstrated minimal allogencity, but also visible degredation [57]. "Pickled" cadaver homografts were also used in both adult and pediatric patients. These grafts were fixed with $4 \%$ formalin, treated with thimerosal, and stored in acetone. During implantation, pickled scaffolds were supported with silicone stents. Bronchoscopic removal of granular tissue was necessary after stent removal, but complete epithelization was observed. Out of 6 patients, none had positive outcomes, 1 died due to an arterial fistula, 2 had recurrent tracheomalacia requiring stents, and 2 underwent stenting with a tracheostomy tubes [58]. Somewhat better results were cited for 18 of 31 children [59]. For all of these pickeld grafts, epithelialization may occur, but cartilage degradation is inevitable, resulting in stenosis and malacia [60].

\subsubsection{Autograft Transplantation}

The inability of non-viable tracheal grafts to encourage host cell infiltration and remodel into a viable tissue presented researchers with few new avenues. Luckily, during the 1950s and 60s there were significant strides in tissue and organ transplantation, including the first 


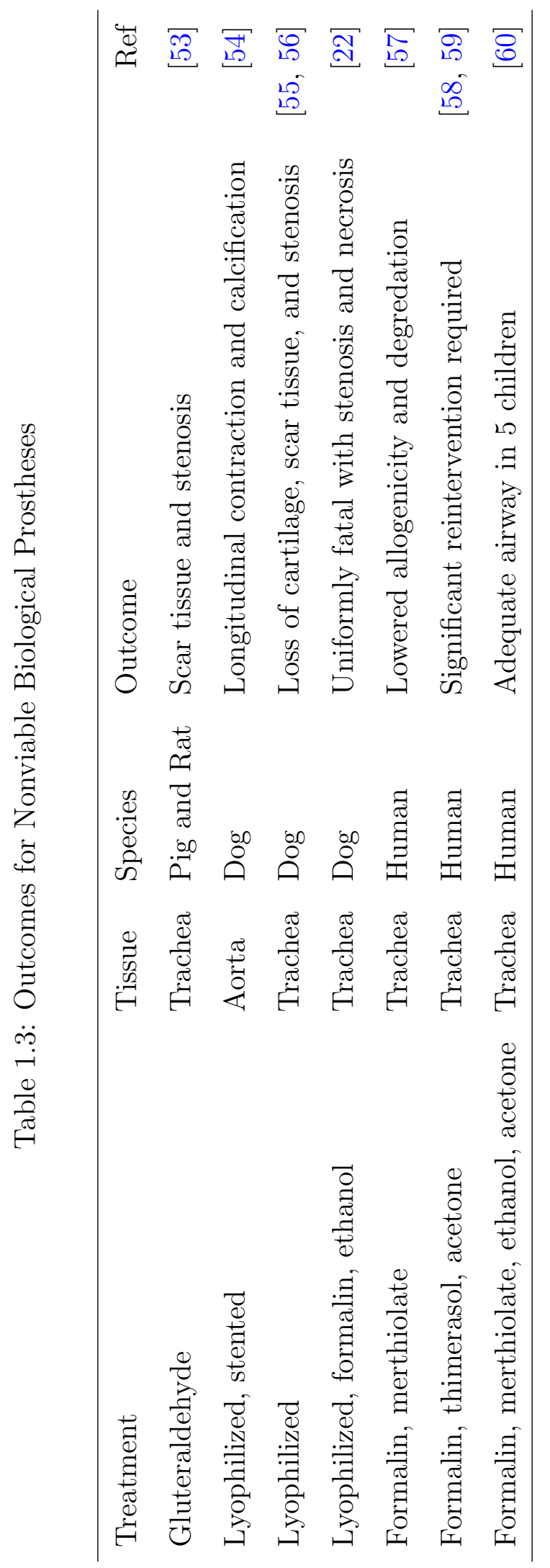


successful kidney, liver, and heart transplants. Coupled with preliminary surgical experience employing autogenous tissues to seal synthetic prostheses during transplantation, there was an obvious next step. Much of the next 20 years was spent on a new wave of transplants composed of free grafts, tissue flaps, and tubes assembled, much like Frankenstein's monster, from a hodge-podge of autogenous tissues.

1.4.3.1 Free Grafts Experimental constructs implanted in dogs, pigs, and rats have used fascia [11, 35, 38, 61], cartilage [13, 62], dermal grafts [14, 56], pericardium [63], free periosteum wrap [64, 65, 66], bone strips [21], stented cartilage and perichondrium [67], costal cartilage, periosteum and rib [68], composite patches of buccal mucosa and auricular cartilage, [69] dura mater with wire [70], bladder mucosa [51, 71, 72, 73], jejunal patches [74], and perichondrium from ear and rib[75]. For all of these attempts, it was found that autogenous patches generally results in fibrotic contraction with patchy epithelization. Clinically, steel coil supported fascia has been employed in malignant tumors resections [26]. Larger dermal grafts for tracheal repair were mostly unsuccessful [51, 76], but limited patch reconstructions were moderately successful [77, 78]. Auricular cartilage [79], free bronchial grafts [80], costal cartilage, and pericardium were attempted. The final two were employed successfully for treatment of long congenital stenosis. Re-epithelization and only occasional necrosis was observed[81, 82, 83, 84]. Cartilage patches mostly resorbed and replaced with mature scar tissue [85]. In all pediatric cases it was noted that somatic growth was reduced [86]. Many of the authors argued that insufficient revascularization was a major obstacle to successful remodeling and further attempts were made to maintain blood perfusion through transplantation of tissue flaps.

1.4.3.2 Tissue Flaps The use of vascularized autografts was achieved through preservation of native vasculature or by anastomosis of a nearby vascular supply. Many studies show that a vascularized patch is less likely to necrose than a free patch. Vascularized repair of window defects have included pedicled intercostal muscle [87], periosteum [64], bronchus[88], and rib transfer with re-anastomosis [89]. Clinically, skin flaps have been used extensively to close cervical tracheal defects. Other flaps such as pedicled intercostal muscle and pleura [90], 
periosteum on a muscular pedicle [91], rotated bronchus [92], pedicled diaphragm [93], and pedicled pericardium supported by HDPE [10] were used. While effective for small defects, none of these readily permitted treatment of long segment stenosis or repair after malignant tumor excision. To accomplish these goals, surgeons fabricated tubes from similar pedicled autogenous tissues reinforced with cartilage or bone.

1.4.3.3 Tubular Constructs Edgerton and Zovickian [51] reviewed early attempts at tubular graft construction. They describe many multi-staged cutaneous tubes used for cervical trachea reconstruction $[94,95,96,97,98,99,100,101,102]$. Other grafts have employed tubular dermis pedicles, supported with rib or costal cartilage and sometimes lined with split grafts. Serrano and colleagues [103] inserted a series of c-shaped cartilage rings carved from costal arch to provide support when a skin trough was closed anteriorly. Papp and colleagues, unsuccessfully, wrapped intercostal muscle, stiffened with cartilage and lined with skin around a stent, in dogs [104]. Krespi and colleagues wrapped a periosteal flap around a silicone elastomer stent and after 6 weeks in a subcutaneous location, moved the tube to an orthotopic location [105]. Kon and Van den Hooff also produced cylinders of cartilage by wrapping rib perichondrium around a silicone rod implanted in tissue [106]. Another line of experimental reconstruction with host tissue has been the use of adjacent esophagus to replace a long segment of trachea. Placement of c-shaped polypropylene rings failed when the adjacent supported segments caused obstruction. Attempting this procedure simply to enclose a silicone t-tube substitutes the challenge of a tracheal repair for an esophageal repair [107]. In an effort to avoid the necessity of a major operation for later esophageal replacement, re-vascularized segments of small intestine have also been used experimentally [108]. Given the lack of mechanical rigidity, it'sunclear how the patient was able to breath. With any of these approaches, the complexity of the construction, in vivo culture, and final surgery can result in fatal mediastinitis. Additionally, for each autograft approach the patient faces significant concerns with graft failure as with non biological approaches, but must then also contend with donor site morbidity. For example in the case of costal cartilage, the most widely used clinical graft, there are numerous reports of resultant pneumothorax (collapsed lung) and pneumonia. Whole organ allografting was attempted 
to avoid the problems with autograft tissue, unfortunately, they suffer from many of same issues and even create new ones.

\subsubsection{Whole Organ Transplantation}

Preliminary research demonstrated that even immediate orthotopic re-implantation of an animals own trachea was unsuccessful because neovascularization is too slow to maintain tissue metabolism [6, 22, 36, 109, 110, 111]. As discussed above, omentum has been used to successfully revascularize autografts tissue effectively allowing the graft to recover from early ischemia [111]. However, even with omental revascularization, canine autografts longer than $4 \mathrm{~cm}$ frequently showed stenosis or dissolution in their central portions [112]. These findings were supported by Balderman who showed that omental wrapping was incapable of sustaining chondrocyte viability in canine long segment tracheal autografts [113]. The totality of these findings conclusively demonstrate the necessity of a blood supply at implant.

1.4.4.1 Revascularization Revascularization of an orthotopic whole tracheal graft has been most sucessful through direct vascular reanastomosis. Unfortunately, due to their small size and distribution, the arterial and venous supply of the trachea do not easily lend themselves to direct revascularization. Researchers have combated this problem through the preparation of thyrotracheal grafts, anastomosing the thyroid artery to the common carotid artery [114]. In the absence of immunosuppressive therapy, cartilage was preserved but tracheal soft tissues necrosed. With cyclosporin and hydrocortisone, all tracheal tissues survived. In a conflicting finding, Macchiarini and colleagues observed preservation of tracheal grafts, including epithelium, in a heterotopic pig model using cyclosporin with both arterial and venous anastomosis [115]. Macchiarini found that venous infarction occurred in the absence of a venous anastomosis [116]. Macchiarini also experimented with thyrotracheoesophageal transplantation, adding perfusion of the arterial system of the graft with a hypothermic flushing solution [117]. Strome and associates described fresh laryngeal transplantation, which included a five-ring segment of trachea, thyroid, and parathyroids, plus

both superior laryngeal nerves to replace a traumatically scarred larynx. Arterial, venous, 
and neural anastomoses was accomplished and perfusion was established. 40 months after surgery,the patient was healthy with functional vocal chords [118]. This one off success has not been repeatable and led to further work assessing the immunosupressive therapeutics and tissue pre-treatment.

1.4.4.2 Implant Modification Fresh tracheal allografts typically initiate a strong, acute immune rejection upon implantation $[119,120,121,122]$. A study by Beigel at al identified antigens within the rodent mucosa which mediate this rejection [120]. This work was further supported by findings that human tracheal mucosa was the major antigenic structure responsible for immunogenic action of tracheal allografts [121]. Combined these studies demonstrated that tracheal transplants are not weakly antigenic as originally posited [123]. Long segment fresh tracheal allografts have only systematically worked when revascularized and in the presence of strong immunosuppresants. [113, 124, 125, 126, 127, 128]. To avoid costly HLA matching, and therapeutic complications, various treatments including cryopreservation and irradiation were attempted to modulate the immune response of allografts. Cryopreservation seems to partially inhibit allogenicity while promoting neovascularization. Cryopreserved allografts with omental flap revascularization survived without immunosuppressive therapy $[124,125,129]$. Mukaida observed gradual replacement of graft epithelium by recipient epithelial cells after sixty days in a cryopreserved tracheal allograft [130]. However, Moriyama and associates found that cryopreservation of allografts reduced acute rejection and permitted early revascularization, but eventually resulted in chronic rejection and atrophy [131]. Tojo and coworkers found that after transplantation, cryopreserved tracheal allografts in rats without immune suppression were colonized by host epithelium, but the chondrocytes were of donor origin [132]. Inutsuka and colleagues noted the feasibility of cryopreservation (for 3 weeks at -80 C) of tracheocarinal allografts in dogs and subsequent viability without immunosuppressive therapy [133]. Throughout these studies the mechanism for the reduced allogenicity due to cryopreservation is unclear. 
Table 1.4: Requirements for a Suitable Tracheal Replacement

General Prosthetic Characteristics

Readily available or minimal fabrication time required

Airtight at implant

Appropriate Mechanical Features - longitudinal compliance and circumferential rigidity

Minimal Inflammatory Response

Incorporate into surrounding tissue, no migration or dislodgement

Support growth and differentiation of respiratory epithelium

Readily vascularized to minimize stenosis and necrosis

\subsubsection{Next Steps}

From the extensive failures it is possible to develop a list of desirable characteristics for a potential tracheal prosthetic (Table 1.4). To date all sucessful surgical prostheses have been implanted within mesenchymal tissue. Within the airway an interface inevitably persists among foreign material, chronically repairing connective tissue, and epithelium, which is a perpetual source of bacterial contamination. Trials of one material after another cannot be expected to solve this basic biological incompatibility. Macchiarini summarized it as: "tracheal allografts necrose whatever the conservation procedure. Only a living substitute, therefore vascularized, can pretend to fulfill the anatomic mechanical and anti-infectious functions of the trachea." The recent advances in fields as diverse as stem cell biology, biomaterials, and biomechanics has made the possibility of a living prosthetic composed of a patients own cells a reality. Tissue engineering, may provide the revolutionary, rather than evolutionary, next step necessary to produce a truly efficacious tracheal graft. 


\subsection{A LIVING PROSTHETIC: TISSUE ENGINEERED TRACHEA}

First described by Langer and Vacanti, Tissue engineering is an: "an interdisciplinary field that applies the principles of engineering and life sciences toward the development of biological substitutes that restore, maintain, or improve tissue function or a whole organ" [134]. In effect, tissue engineering harnesses the body's ability to regenerate through the appropriate application of mechanical and biological cues. In native, healthy tissue, cells are interspersed in a complex network of fibrous proteins and proteoglycans. This extracellular matrix (ECM) provides 3-dimensional support and organization to the cells. They exist in a symbiotic relationship, cells respond to biological signals contained within the ECM and in turn alter the organization and composition of the ECM. An external factor such as an injury can perturb the system, resulting in changes to both the cells and ECM. When a foreign object is implanted into the body, these same interactions take place, essentially the structure, mechanics, and biology of these objects dictate the host reponse. Tisue engineering aims to design an implant to mimic the native biological milieu and encourage host cells to assume an appropriate phenotype. Several pioneers demonstrated reserach culminating in a tissue engineered human tracheal transplants. The first paper reported on a decellularized tracheal allotransplant which was repopulated ex vivo by use of a complex air-liquid bioreactor. The second involved a synthetic tracheal prosthesis that had been repopulated by use of an in vivo tissue-engineering technique. The third paper switched to a decellularised allotransplant with in vivo repopulation. However, the working mechanism of the tissue engineered airway remains unclear. The sucess of these three trial studies, conducted under compassionate use, has prompted pre-clinical work to lay the foundation for future, controlled human trials There have been many approaches, but in general, researchers employ a porous scaffold which supports and directs the cellular population. These scaffolds may be synthetic or biologically derived, but are mostly designed to be readily resorbed in vivo. These constructs may be seeded with one or more cell types, precultured prior to implantation, or even in some cases implanted without cells. 


\subsubsection{Synthetic and Biologically Derived Polymers}

The first generation of tissue engineered devices were mostly synthetic. As with any synthetic material, biocompatibility and immune response of the polymer and it's degredation products are a significant concern. To this end, initially only well characterized polymers were attempted. They were fabricated through a variety of techniques, including electrospinning, weaving, felting, TIPS, and salt leaching. The polymers were mostly designed to be hydrolytically degraded in vivo into easily removed fragments. Materials used include polyglycolic acid (PGA) [135, 136, 137], polylactic acid and polyglycolic acid mixture (PLA/PGA) and poly(lacticcoglycolic acid) PLGA [138], polyester urethane [139], poly(ethylene oxide)terephthalate/poly(butylene terephthalate)(PEOT/PBT) [140, 141], and gelatin sponge [142]. Vacanti et al. observed no immune response to PGA in nude mice [137]; however, Kojima et al. observed poor cartilage formation in the same PGA constructs in sheep after 7 days, which the authors concluded was likely due to an inflammatory response [136]. A similar immune response was observed in PLA/PGA constructs in rabbits [138]. Pre-culturing the PLA/PGA construct for 2 weeks prior to implantation improved the in vivo response. The authors hypothesized this was due to immune modulation caused by cultured cells preventing blood entering the construct [138]. Further work was conducted using naturally inspired materials. For example, hyaluronan-based scaffolds were been shown to support chondrocyte growth in bioreactor culture [143], however, in vivo rabbit implantation of allogeneic chondrocyte-seeded hyaluronan-based scaffolds (12 weeks) caused a nonspecific foreign body response and eventual degradation of tissue-engineered cartilage [144]. As this reaction is not observed in knee articular cartilage, the authors hypothesized that the vascularity in the neck may create an unfavorable immune microenvironment. Silk fibroin has also been shown to be biocompatible in rabbit tracheas for 12 weeks, with fibroblasts and capillary vessels integrating into the material [145].

\subsubsection{Decellularized Tissue}

After removal of cellular antigens and debris by decellularization, ECM grafts retain a variety of proteins including, growth factors, proteoglycans, and other matrix proteins in a 
mostly native configuration. ECM grafts mimic the unique structure and function of each tissue, providing potent cues to infiltrating host cells. Additionally, when degraded in vivo they release peptides that possess bioactivity distinct from that of the parent molecule, including bacteriostasis, chemotaxis, and mitogenesis. Thus, decellularized tissue scaffolds have been used in multiple areas of tissue engineering. Most importantly, Macchiarini et al. showed that decellularized human trachea could also be used in a clinical tissue engineering paradigm [146]. Decellularized aortic grafts[147], jejunum [148, 149, 150], bladder [151], and trachea $[151,152,153,146]$ have been utilized for tracheal replacement. These scaffolds have been used with or without cells; however, Go et al. observed contamination and infection in constructs not seeded with epithelial cells after 11 days in a swine model [152]. Remlinger et al. observed scaffold degradation after implantation of hydrated xenogeneic decellularized trachea extracellular matrix in dog tracheas for 8 weeks [154]. For future studies, the authors suggest adding chondrocytes to the scaffolds, with the goal of replacing the degrading scaffold with cartilage. Preservation methods of such scaffolds also seem to have an effect on regeneration. Gilbert et al. did not observe any cartilage formation in their decellularized trachea and bladder constructs when implanted in dogs for 6 months, and they hypothesized this may possibly have been due to lyophilization and freeze-drying of their construct before implantation [151].

\subsubsection{Cell Lines in Tracheal Tissue Engineering}

From literature it is clear that the success of a tracheal graft depends on it's structure, mechanics and antigenicity, however, the cellular component must not be overlooked. Various groups have attempted to seed a range of cell types, from chondrocytes to mesenchymal stem cells, obtained from a number of donor tissues. Three main strategies have been undertaken: acellular, single, and multiple cell types.

1.5.3.1 Acellular Constructs Acellular constructs rely on the biomaterial and any added growth factors to harness the bodys ability to allow cells to populate and integrate into the material. The influence of growth factors on tissue ingrowth is significantly more 
important for acellular constructs and has been investigated with some promising results $[142,155]$. However, it is unlikely that this approach can be successful clinically since all cells must migrate into the material from the surrounding native tissue. This strategy is most often used for decellularized materials as a proof of concept since the necessary growth factors to modulate immune response and trigger a positive healing response are present at implant.

1.5.3.2 Single Cell Type To date, tracheal research has primarily employed a single cell type strategy. Chondrocytes were used in Vacantis first attempt at tracheal tissue engineering [137] and have been used extensively since. It is clear from the many failures, that creating or maintaining functional cartilage is critical to the most basic function of the trachea [156]. While native epithelial cells have been shown to migrate onto the lumen of tissue-engineered constructs, full reepithelialization takes considerably longer. For large segment reconstruction, full epithelialization occurs slowly, if at all. Nevertheless, it is clear that an intact epithelium is vital to bacterial resistance, prevention of granulation tissue and stenosis [157]. The source of chondrocytes and epithelial cells is crucial to this end. While an autologous source is preffered to limit antigencity, donor site morbidity is a concern. Human tracheal chondrocytes may have the chondrogenic potential to form tissueengineered cartilage for clinical use [158]. Unfortunately, obtaining tracheal chondrocytes is invasive, so the clinical relevance is limited. Comparisons of chondrocyte sources (nasal, articular, auricular, tracheal, and costal) [143, 159, 149], indicate that donor site morbidity seems to be the only difference. Ideally, it would be possible to easily obtain a single cell type capable of differentiating into the multiple required cell types. Adipose-derived stem cells [160], bone marrow stromal cells [161, 162, 146, 163, 150, 164], and amniocytes [165] have been utilized for chondrogenesis in the tissue engineered trachea. Cell sources for airway epithelium include respiratory tract endogenous cells and exogenous cells from other tissues in the body (embryonic, bonewalles marrow, amniotic fluid, etc.) [166]. For example, fully differentiated airway epithelium has been generated from embryonic stem cells through an in vitro culture process [167]. Increased research into stem cell technologies such as iPS presents a new avenue for tracheal tissue engineering. However, regulatory challenges will 
likely postpone their introduction into mainstream use, thus the leading cell source is most likely the patients own bone marrow mesenchymal cells.

1.5.3.3 Multiple Cell Types The most complex approach involves the seeding of multiple cell types, either simultaneously or serially. Prior to adding the cells to the scaffolding, researchers have cultured the cells together or separately. One study evaluated the performance of epithelial cells on chondrocyte pellets, epithelial cells on native cartilage explants, and coculture on opposite sides of collagen membranes [168]. Immunostaining staining revealed expansion and differentiation in the epithelial layer only is the final method. The authors concluded a basal lamina equivalent, fibroblast conditioned medium, and air-liquid interface were required for epithelial cell proliferation and differentiation. Another group cultured human respiratory epithelial cells and chondrocytes separately on 3D scaffolds, and then fused the two constructs together with fibrin surgical adhesive. After 5 days in culture, the constructs remained viable and the layers were appropriately adhered to each other [169]. Chondrocyte and epithelial cell constructs have been seeded abluminally and luminally, respectively. These constructs have been introduced into subcutaneous pockets of nude mice $[170,171]$. At the conclusion of 6 weeks, the ECM content of the tissue-engineered cartilage was similar to native cartilage, and histology revealed mature cartilage and pseudostratified columnar epithelium [170]. The function of these constructs must still be evaluated in vivo. However, this biomimetic seeding strategy has had some success in humans, when seeding chondrocytes differentiated from MSCs on the exterior surface and epithelial cells on the interior surface[146]. Other combinations of cells utilized for trachea repair are epithelial cells and fibroblasts [172], endothelial cells and epithelial cells [173], chondrocytes and fibroblasts [136], fibroblasts and muscle cells [148], and bone marrow stromal cell-derived chondrocytes and epithelial cells $[152,146]$. Walles et al. evaluated an even greater number of cell types; seeding chondrocytes, bone marrow-derived smooth muscle cells, bone marrowderived endothelial cells, or respiratory epithelium on a decellularized matrix[150]. More research is needed to determine whether multiple cell types can be incorporated onto one construct at one time and whether this approach is clinically beneficial or practical (i.e., multiple biopsy sites). 


\subsubsection{In Vitro Culture}

Once cells are seeded on a scaffold, the constructs can then be exposed to various in vitro culture before implantation. Bioreactor and static culture have been used independently and in conjunction. One group showed that bioreactor versus static culture did not have an effect on the GAG content and mechanical properties of scaffold-free chondrocyte constructs [174]. Luo et al. suggested that in vitro preculture of constructs may reduce inflammation of constructs and promote better ECM formation after implantation in vivo [138]. Another example employed a chondrocyte seeded collagen/PCL tube in a rotating wall bioreactor in vitro, followed by orthotopic implantation in rabbits, unfortunately, granulation tissue caused stenosis in all animals [138].

\subsubsection{Surgical Implantation}

While constructs have been extensively evaluated in static or dynamic in vitro culture, ultimately they must be placed in an in vivo model to determine functionality and performance in a living organism. Animal models used for tracheal tissue engineering vary from mice to pigs, but as of yet a standard animal model has not been established [175]. The size and location of implantation also differs. Some studies implanted the tissue-engineered trachea subcutaneously [159]. In these cases, survival rates were usually high and tissue in-growth was observed; however, the functionality of the construct cannot be analyzed with subcutaneous implantation. A majority of the tracheal tissue engineering studies placed the construct in a patch or circumferential defect in the trachea. Survival rate and performance of the constructs were typically good in patch (or window) defects, while lower survival rates and more complications occurred in circumferential defect repair. Interestingly, some researchers have implanted grafts in a heterotopic location to harness the body as an in vivo bioreactor prior to final implant. One approach implanted the graft subcutaneously to precondition the construct, and then transplanted the construct orthotopically[176]. An intricate approach was recently introduced, where tracheal constructs were cultured in a perfusion bioreactor in vitro, followed by paratracheal implantation, and finally implanted orthotopically [177]. This complex approach was an abject failure, stenosis was observed in all cases, potentially due to the lack of an established endothelial lining. 


\subsubsection{Hurdles to a Tissue Engineered Trachea}

Incredible time and effort has been invested into developing a tracheal prosthetic, the tissue engineered constructs developed so far demonstrate promise for a clinically useful tissueengineered replacement. However, many problems have arisen, such as how to maintain structural support of the tissue-engineered construct, controlling the scaffold degredation, minimizing immune mediated rejection, optimizing re-epithelialization, and promoting revascularization. Even the first clinically transplanted tissue-engineered trachea, which has functioned successfully in patients thus far, was limited by a long-preparation time, and reliance on donor tissue. With that being said, current therapeutics are a necessary stop gap measure, helping patients with the most devastating tracheal diseases. The reliance on donor tissue, however, for the decellularized scaffolds is a major limitation, and a xenogeneic scaffold is a better long-term goal. In addition, the biological activity of a biological matrix is a doubleedged sword, capable of promoting positive remodeling or catalyzing acute inflammation and rejection. Since decellularization dramatically alters tissue structure, mechanics, and composition, it is vitally important to unravel the effect of decellularization on ECM structure and biology gaining insight into the host response to better engineer a viable tracheal replacement.

\subsection{RESEARCH GAP \& SPECIFIC APPROACH}

Tracheal repair procedures were first attempted in the late 19th century and after more than one hundred years of research; no curative treatments for large tracheal defects have been established. The use of prosthetic materials, stents, tissue flaps, autografts, or a combination of these methods have been reported, but complications are pervasive: migration, dislodgement, material degradation/failure, bacterial infection, granulation tissue, stenosis, necrosis, anastomosis failure, erosion of major blood vessels, need of life-long immunosuppression, lack

of appropriate donor sources, and lack of adequate vascularization. For example, primary tracheal cancers result in neoplastic lesions, which are often not diagnosed until the tumor 
exceeds $6 \mathrm{~cm}$ of the tracheal length and becomes inoperable. These patients are palliated, but have a poor prognosis, with reports of five-year survival between $5 \%$ and $13 \%$. Thus, there is a clear and present need for improvement in the treatment of severe congenital and acquired tracheal defects; tissue engineering presents the most promising avenue to create safe and efficacious tracheal graft. Preliminary studies have demonstrated the use of decellularized human trachea clinically with excellent short-term results [148]. In this approach, cellular material was removed from a human donor trachea with detergent and enzymatic rinses. The remaining extracellular matrix (ECM), was then reseeded with the patients own cells and implanted. Wider adoption of this approach has been hampered by significant donor tissue limitations. Since xenogeneic ECM are well tolerated during transplant due to conservation between species, the goal of this application is to engineer a decellularized porcine trachea suitable for human transplantation. We hypothesize that the ECM scaffold features are determined by tissue processing parameters, which in turn direct the host response. Further, we believe that by more fully characterizing the effect of detergent based decellularization on in vivo biological function in a small animal model; we may ultimately develop a better porcine trachea decellularization protocol resulting in a safer and more efficacious tracheal graft. To accomplish this goal, three specific aims are addressed.

Specific Aim 1 Characterize the effect of alternate rat tracheal decellularization protocols in terms of structural and compositional changes.

Specific Aim 2 Test the functional tissue remodeling potential of the previously characterized decellularized trachea in an established orthotopic rat tracheal transplant model. Assess host mediated remodeling through clinical and functional metrics including scaffold recellularization and epithelial differentiation.

Specific Aim 3 Translate findings from aims one and two to a clinically relevant xenogeneic scaffold. The scaffold will be validated through mechanical testing and it's ability to support human epithelial cells in vitro. 


\subsection{CHARACTERIZATION OF DECELLULARIZED RAT TRACHEA}

\subsection{INTRODUCTION}

When employed for tissue engineering, extracellular matrix (ECM) scaffolds are desirable for many reasons. They are readily repopulated by host cells, degraded in vivo and replaced with de novo tissue deposited by the invading host cell population. Unlike polymeric scaffolds, they also contain remnant proteins and signaling molecules, which can provide very potent cues for regeneration. These decellularized matrices may be obtained through treatment with detergents, acids/bases, enzymes, alcohol, or even mechanical disruption. Unfortunately, there is no universal protocol, the most effective decellularization agents must be identified for each tissue due to the specificity of structure, composition and geometry. It is generally accepted that no tissue may be decellularized without disruption of the native structure. Therefore, given our current tissue engineering paradigm the minimization of these perturbations must be the goal. Recently, researchers have begun to explore the transplantation of tissue engineered whole organs $[178,179]$. The translation from a single tissue to a larger, more complex structure has created many new challenges. Nevertheless, great strides have been made. Due to it's relative simplicity compared to a heart, liver, or lungs, the trachea was one of the first clinical applications of a whole organ tissue engineering. Preliminary clinical studies have employed a decellularized human trachea graft to engineer a living prosthesis [148]. Despite the early clinical success there remain many outstanding questions. For example, it's clear that the decellularization approach is vitally important, yet there has been surprisingly little preclinical work justifying their particular selection. Instead it seems the authors simply translated the first working protocol. The current clinical protocol, the detergent enzymatic method (DEM), employs repeated treatments with 
detergent washes and enzymatic DNA degradation. In general, detergents are the most commonly utilized decellularization agents due to their unique amphilic properties. Amphiphiles are interesting molecules which possess both polar and non-polar characteristics. Typically they are long chains containing a bulky polar head group and a non polar tail. In aqueous solution they form micelles, where the detergent molecules self assemble into spheroids to minimize their surface energy. In a micelle all of the non polar tails face inwards and the polar head groups shield them from the surrounding aqueous solvent. These micelles are able to diffuse into a tissue, whereby individual detergent molecules are able to integrate into the phospholipid bilayer of the cell membrane. Due to the smaller tail groups, these detergent molecules increase the flexibility of the cell membrane and eventually cause the cell to rupture. After lysis, remnant phospholipids from the cell membrane and membrane bound non polar proteins are solubilized through incorporation into the detergent micelles.

While there are an enormous number of different detergents, they are most often categorized into three types. Ionic, meaning they have a permanent charge, non-ionic those without, and zwitter ionic, those that have an equilibrium state with both charged and uncharged states. In general ionic detergents are considered to be more harsh, they have strong monomer affinity to proteins, the non polar tail can bind to non polar regions of the protein, which alters the tertiary folding. This in turn allows more detergents to bind, known as cooperative binding, which can completely denature the higher order structure of the protein and effectively destroy it's biological function. For example, sodium deoxycholate, the detergent employed clinically, is often used to denature proteins prior to enzymatic degradation for proteomic analysis [180]. Ionic detergent monomer affinity also makes it harder to remove residual detergent from a decellularized tissue after processing. Non-ionic and zwitter ionic detergents are considered to be less harsh, they are typically non-denaturing and are often used for isolation of biologically active proteins. Triton X-100 and CHAPS are common examples of non-ionic and zwitter ionic detergents respectively. In the current clinical paradigm, Macchiarini et al employ repeated washes with sodium deoxycholate, a harsh ionic detergent, over six weeks to effect decellularization. Many previous experimental studies have attempted sodium deoxycholate decellularization on a host of different tissues, including cornea [181], urinary bladder [182], ligament [183], and peripheral nerve [184]. 
These publications have reported mixed results in vivo, but generally have shown extensive ECM disruption. We hypothesize that this prolonged deoxycholate exposure unnecessarily damages the trachea and that alternative decellularization agents may result in more effective tissue engineering scaffold. Therefore, we propose to evaluate three alternate, commonly employed detergents, CHAPS, Triton X-100, and SDS. In addition to traditional detergent decellularization, we also propose to test peracetic acid (PAA), a commonly employed agent. Previous work has shown that PAA effectively removes cells and remnant DNA while having a minimal effect on ECM composition and structure[185, 186, 187]. However, given the broad applicability and success in the decellularization of membranes and thin planar tissues, there is surprisingly little quantitative data on PAA's benefit when compared to detergent based decellularization in larger, denser tissues. Oblique references in literature and anecdotal evidence suggests that it may improve the in vivo response of scaffolds. We wish to specifically answer this question by directly comparing tracheas processed solely with the detergent enzymatic method to those that are identically processed, but additionally receive a final PAA rinse. We further hypothesize that PAA treatment will have a minimal effect on scaffold properties. In this aim, all decellularized scaffolds will be thoroughly assessed to identify morphological, histological, and compositional differences resulting from the processing.

\subsection{MATERIALS \& METHODS}

\subsubsection{Animals}

All animal experiments were reviewed and approved by the University of Pittsburgh Institutional Animal Care and Use Committee and were performed in compliance with the Guide for the Care and Use of Laboratory Animals as published by the NIH. Age-matched female Lewis rats (approximately $150 \mathrm{~g}$ in weight) were used in the study as donors. 
2.2.1.1 Tissue Collection Lewis rats were anesthetized with a cocktail of ketamine hydrochloride $(100 \mathrm{mg} / \mathrm{kg})$ and xylazine hydrochloride $(10 \mathrm{mg} / \mathrm{kg})$ administered intraperitoneally. An incision was made in the abdominal cavity and the abdominal aorta was transected to exsanguinate the animal. The entire length of the trachea was exposed via a sternotomy with concomitant midline cervical incision. The trachea from the larynx to just proximal to the bifurcation was excised with the use of a surgical microscope.

\subsubsection{Scaffold Preparation}

After explantation, specimens were thoroughly rinsed with deionized (DI) water and cleaned of extraneous tissue under a Zeiss DV4 dissecting scope. Samples were frozen at $-80^{\circ} \mathrm{C}$ until use. Specimens were categorized into one of four detergent groups: $3 \%$ triton X100, 1\% SDS, 8mM CHAPS, or 4\% Sodium Deoxycholate. Tracheas were then exposed to a nine day decellularization protocol. Cycles consisted of treatment for 2 hours on a rocker at room temperature with their associated detergent. Tracheas were thoroughly rinsed and treated with 2,000 KU DNAse-I in 1M saline for 3 hours at room temperature on a rocker. Specimens were then rinsed in phosphate buffered saline (PBS) with 1\% antibioticantimycotic (anti/anti, Gibco Life Technologies) for 10 minutes and stored overnight in PBS with $1 \%$ anti/anti. This process was repeated 8 times. Half of the specimens from Triton X-100 and sodium deoxycholate detergent groups were exposed to a single $0.1 \%$ PAA and $4 \%$ ethanol treatment for 90 minutes followed by three 30 minutes rinses in PBS. Finally, all trachea were individually packaged in physiologic saline and gamma irradiated to ensure terminal sterilization. (Figure 2.1)

\subsubsection{Differential Scanning Calorimetry}

Decellularized tracheal matrices (minimum $\mathrm{n}=5$ ) in PBS were blot-dried with Kimwipes (Kimberly-Clark, Neenah, WI) to remove excessive surface water. Samples were hermetically sealed in Tzero crucibles (TA Instruments, New Castle, DE), loaded onto Q2000 differential scanning calorimeter (TA Instruments, New Castle, DE) between $20^{\circ} \mathrm{C}$ and $30^{\circ} \mathrm{C}$. Specimens were then pre-cooled at $3^{\circ} \mathrm{C} / \mathrm{min}$ to $2^{\circ} \mathrm{C}$ before samples were scanned at $3^{\circ} \mathrm{C} / \mathrm{min}$ up to $125^{\circ} \mathrm{C}$ 


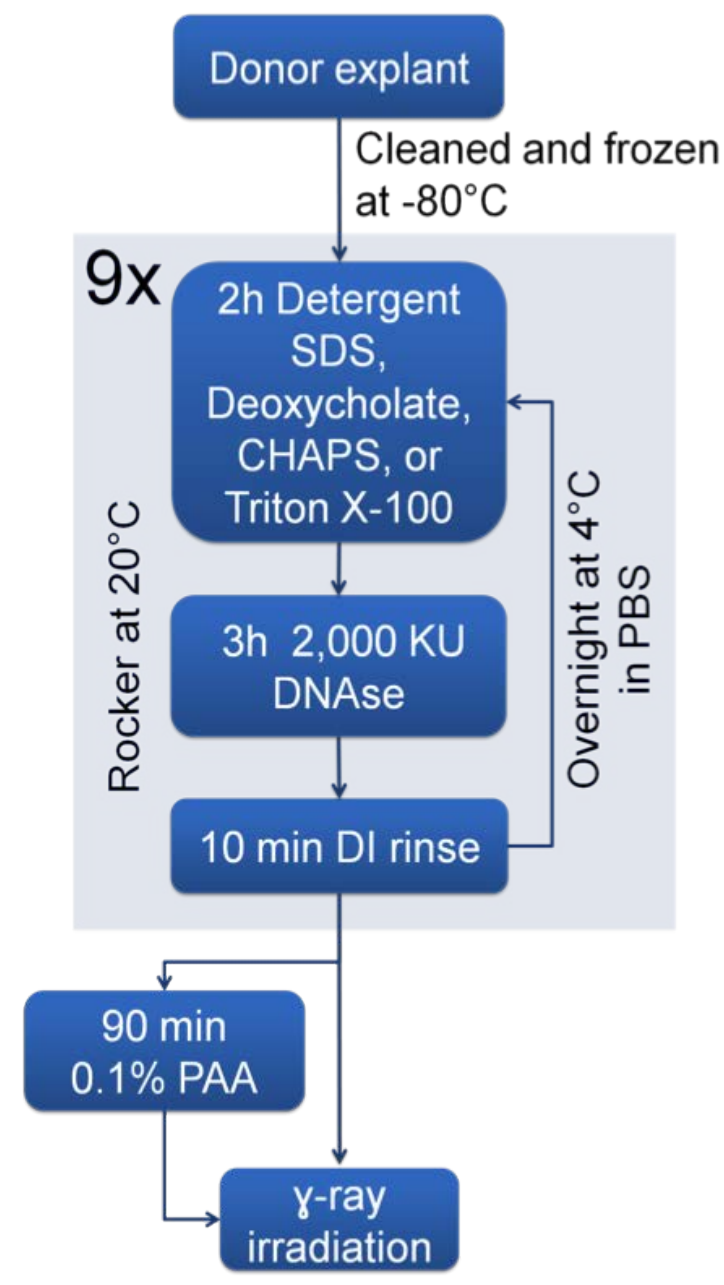

Figure 2.1: Decellularization schematic derived from the clinically accepted detergent enzymatic method. Each day specimens were subjected to two hours in the specified detergent and three hours in $1 \mathrm{M}$ sodium chloride with 2,000 KU DNAse I with gentle agitation at $20^{\circ} \mathrm{C}$. Scaffolds were rinsed and stored overnight in PBS with $1 \%$ antibiotic/antimycotic. This process was repeated for nine days. Scaffolds were then individually packaged and subjected to terminal gamma ray sterilization 
for thermo-physical analysis under a pure nitrogen purge flow rate of $50 \mathrm{~mL}$ per min. After scanning, small holes were punched on the lids of crucibles and samples were dried in a vacuum oven at $105^{\circ} \mathrm{C}$ for at least for 4 hours to determine dry mass. Data were analyzed with the Universal Analysis software (Version 4.5A). Thermograms were obtained through background subtraction. Relevant quantitative values for total enthalpy, main peak temperature and main peak shoulder onset temperature were computer for each thermogram.

\subsubsection{Pressure Diameter Testing}

The effects of decellularization on the mechanical behavior of decellularized rodent trachea was assessed by pressure diameter testing before and after decellularization. A custom built mecahnical testing system based upon previously published devices was used to evaluate tracheal pressure-diameter response [188, 189, 190]. The pressure-diameter testing system was comprised of a testing chamber, adjustable adapters machined to accommodate rat trachea, an optical LED micrometer, pressure sensor, and syringe pump (Figure 2.2). The system was controlled via a custom designed LabVIEW program (National Instruments v8.6.1, Austin, TX). Each trachea was first submerged in the 3L saline tank and retained on the notched adapter with silk ligature. Upon mounting, a small tension was placed on the tissue along the length of the graft to simulate physiologic tension. The luminal pressure was increased or decreased by pumping air into or out of the trachea using a $60 \mathrm{ml}$ syringe connected to a syringe pump (Harvard Apparatus, Model 11 Plus, Philadelphia, PA). A downstream pressure sensor (Honeywell Sensotec Model FPA, Columbus, OH) was monitored with a USB DAQ card (National Instruments, Austin, TX). An optical LED micrometer (Keyence Model LS 7070MT, Wood Dale, IL) positioned orthogonal to the sample was used to measure the outer diameter (OD) of the trachea continuously as the pressure was varied. Diameter measurements were made in the center region of the tracheal tissue. Tracheas underwent 10 preconditioning cycles at $20 \%$ of maximal load, from $-6 \mathrm{mmHg}$ to $12 \mathrm{mmHg}$ prior to testing. Trachea were then tested for 10 cycles from $-30 \mathrm{mmHg}$ to $60 \mathrm{mmHg}$ at flow rate of $4 \mathrm{ml} / \mathrm{min}$. Custom matlab code automatically identified each cycle, aligns each cycle's extrema, and computes a binned average (Figure 2.3). 


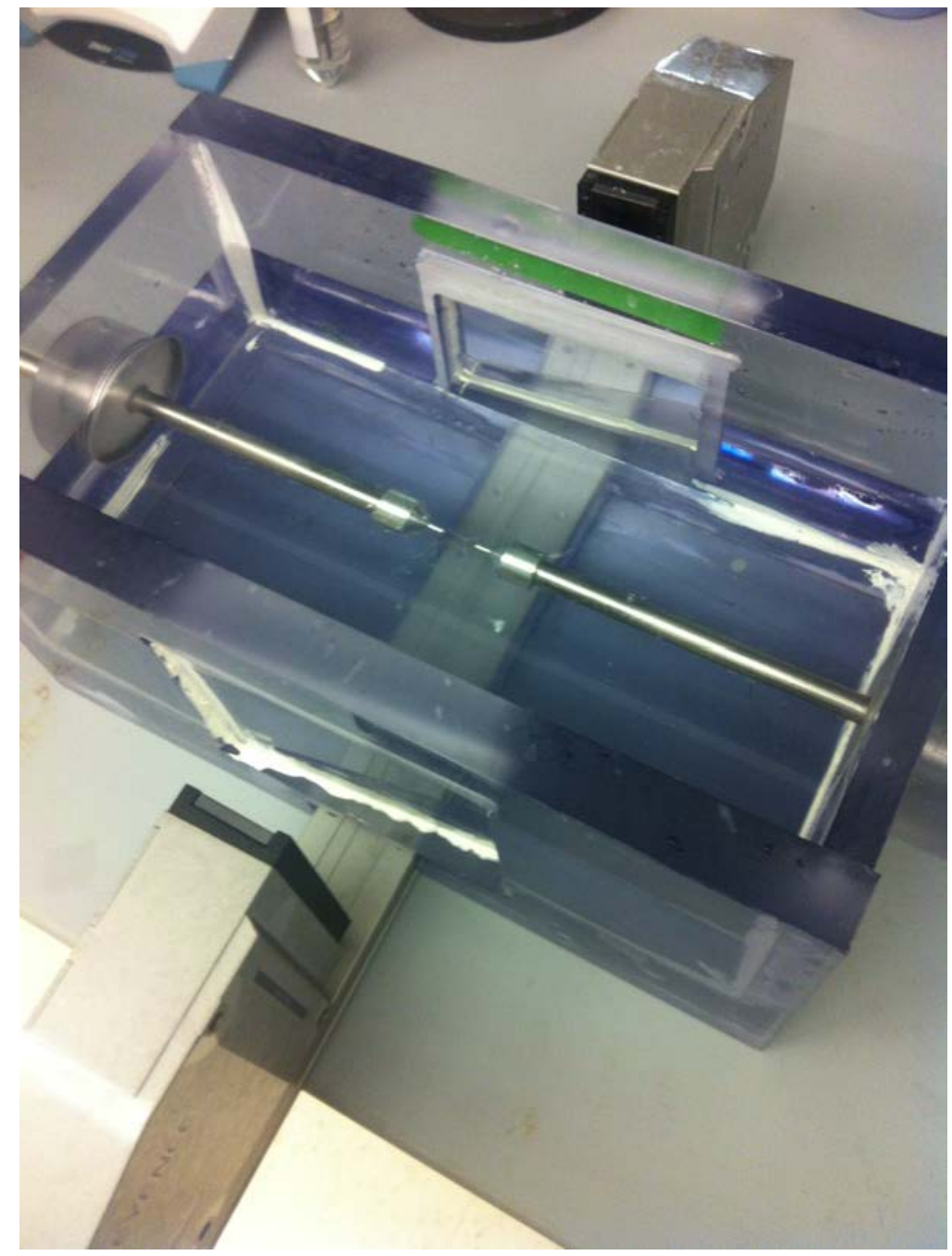

Figure 2.2: A custom built device designed to simultaneously test the pressure and diameter response of tracheas under physiologically relevant levels of air loading. 

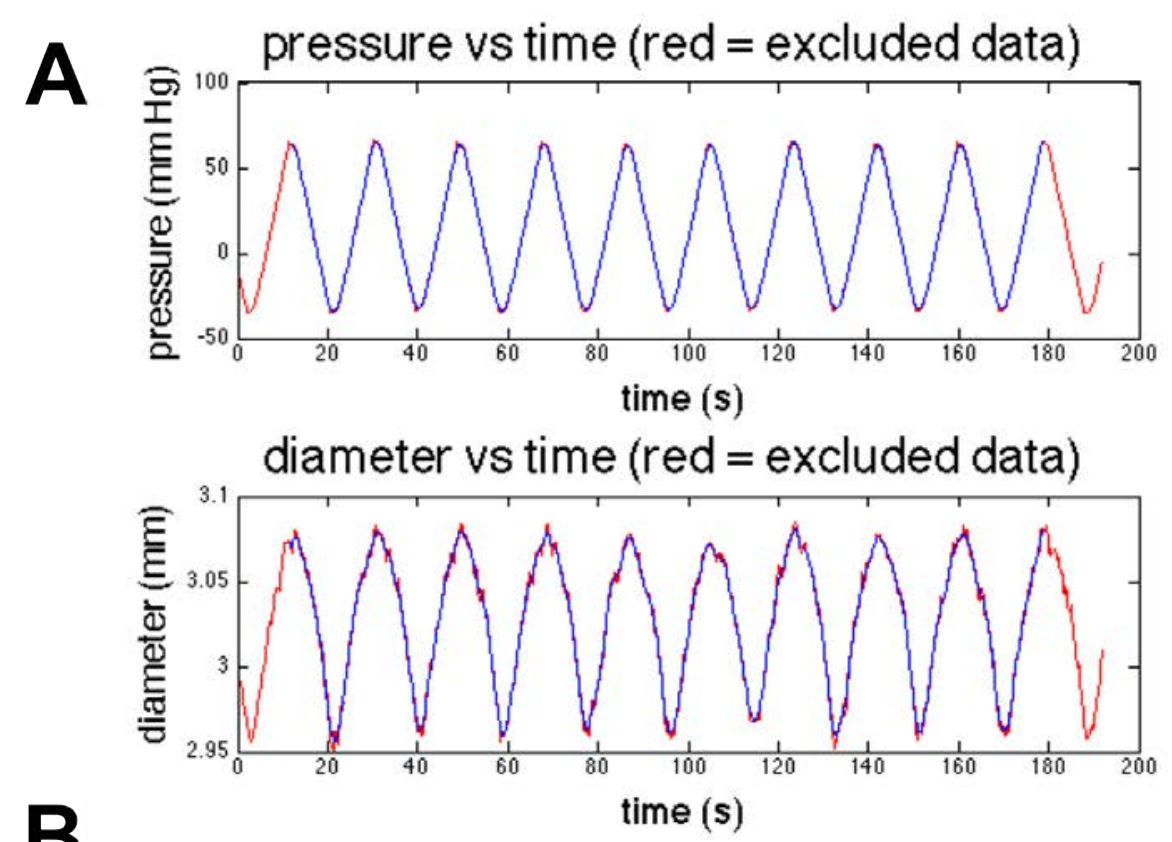

B
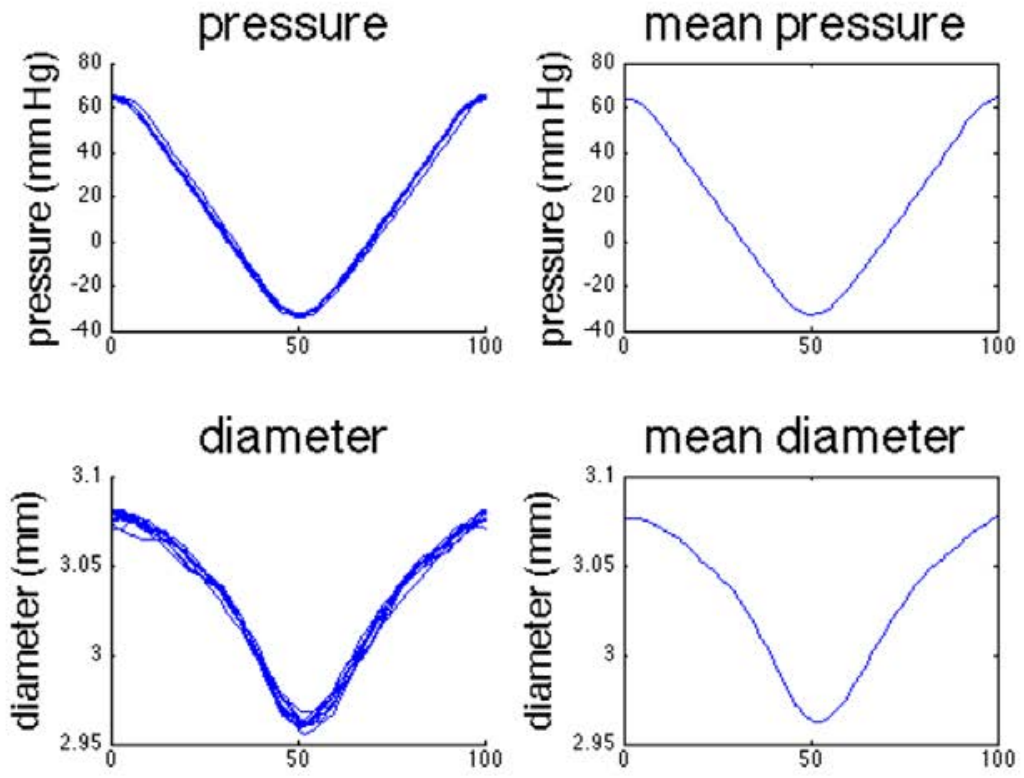

Figure 2.3: Screenshot from custom matlab code. A: Automated cycle identification and data smoothing, excluded data is shown in red. B: Extraction of average pressure and diameter curves. 


\subsubsection{Histology}

Traditional histology was conducted on $10 \%$ neutral buffered formalin fixed and paraffin embedded specimens. Movats Pentachrome was employed to visualize fiber structure, Alcian blue to evaluate glycoaminoglycan retention within the cartilage, and Herovici's to assess collagen cross-linking and relative maturity.

\subsubsection{Statistics}

Statistical analysis was conducted using a multivariate analysis of variance (MANOVA) employing Tukey's post hoc test. For values lacking a subset data point (DSC main peak measurements), one way ANOVA with Tukey's correction was employed. Statistical significance was gained at $\mathrm{p}<0.05$.

\subsection{RESULTS AND DISCUSSION}

\subsubsection{All Treatment Groups Effectively Decellularize Rat Trachea}

All groups, including PAA treated specimens, maintained organ shape and structure, but appeared translucent, consistent with successful decellularization. The thyroid cartilage and larynx often appeared to be incompletely decellularized, but this tissue was dissected away and discarded prior to gamma irradiation. CHAPS and deoxycholate specimens appeared slightly yellow, the mechanism for the odd coloration is unclear (Figure 2.4). After nine, 5 hour treatment cycles with detergent and DNAse I the tracheal mucosa is fully decellularized. Hematoxylin and eosin (H\&E) staining demonstrates complete removal of the epithelial layer and absence of cells and nuclear debris in the cartilaginous segments of the tracheal wall. However, minimal nuclear material was maintained within the condrocyte lacunae of the cartilaginous rings. Cartilage has been shown to be highly immune privileged due to it's denisty and lack of blood supply $[191,192]$. As the mucosa is major antigenic structures within the trachea, we did not believe this minimal residual DNA would negatively impact 
implants. PAA seems to have minimal effect on gross appearance, except for a slight chemical bleaching effect on the deoxycholate specimens. There did not appear to be any observable tactile differences in either circumferential compliance or longitudinal compliance among any of the groups.

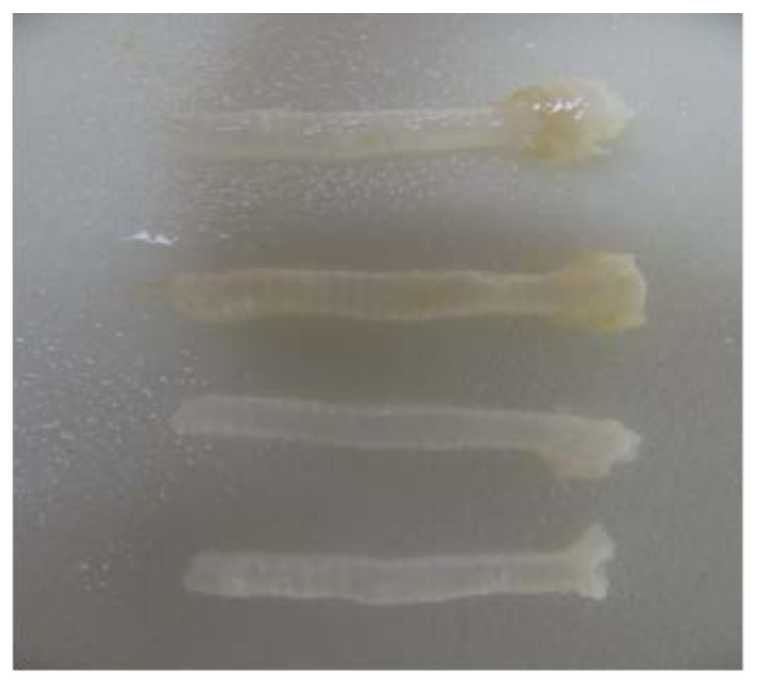

\title{
CHAPS
}

Deoxycholate

SDS

\author{
Triton
}

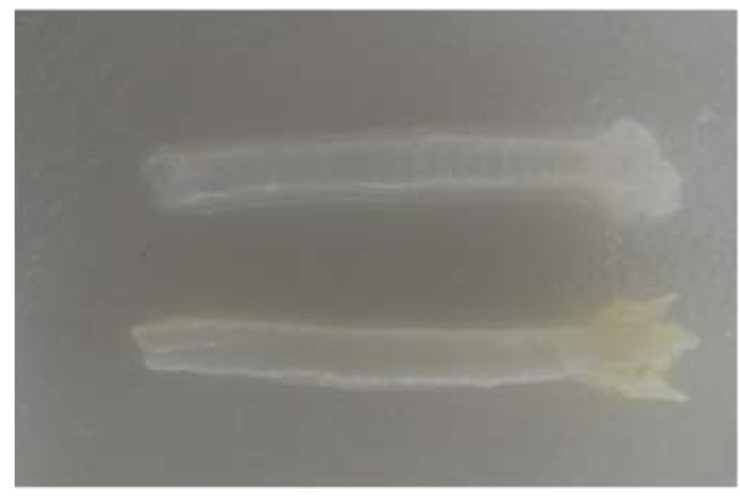

\author{
Triton + PAA
}

Deoxycholate + PAA

Figure 2.4: Gross morphology of rat tracheas after DEM decellularization prior to gamma sterilization. Decellularization preserved organ level structure and shape. Translucent appearance suggests successful decellularization. 


\subsubsection{Cartilage Mechanics, Structure and Composition are Maintained}

The airway performs a critical mechanical role for which a mechanically and structurally sound cartilage is vital. Upon implantation a trachea must operate under full physiological loading immediately. Therefore, to evaluate the retention of native cartilage mechanics and structure, Alcian blue staining and pressure diameter testing were employed. From stained sections it is clear that glycoaminoglycans (GAGs) are maintained within the hyaline cartilage of all decellularized scaffolds (Figure 2.5 and 2.6). Additionally, decellularization appears to have minimal effect on cartilage structure. Decreased staining intensity was observed in PAA treated specimens, but this is likely this is due to histological processing differences as opposed to further removal of GAGs by PAA. One limitation of Alcian blue staining must be considered, it is a highly sensitive stain as even small amounts of GAGs will stain intensely. Therefore, staining is typically used assess structure as opposed to qualitatively or quantitatively assessing GAG content [193].

\subsubsection{Trachea Mucosal Structure is Maintained Despite Cellular Removal}

Contrary to original findings, the mucosa of the trachea is the predominant immunogenic structure $[123,120,121]$. For this reason complete removal of the epithelial layer is vital, however, presence of an intact basement membrane is extremely important to epithelial reconolonization after implant. From the Movat's pentachrome staining, we can see that Triton and CHAPS appear to retain a basement membrane in a generally native configuration (Figure 2.7). SDS and to less of an extent, deoxycholate, demonstrate some removal of the basement membrane and associated elastic fibers. This finding is consistent with our hypothesis that ionic detergents will have greater impact on ECM structure and composition. PAA acid treated specimens demonstrated basement membrane and elastic fibers similar to

their non PAA treated counterparts (Figure 2.8). However, collagen and muscle staining was conspicuously absent. It is not immediately clear whether this is as a result of histology or processing. 

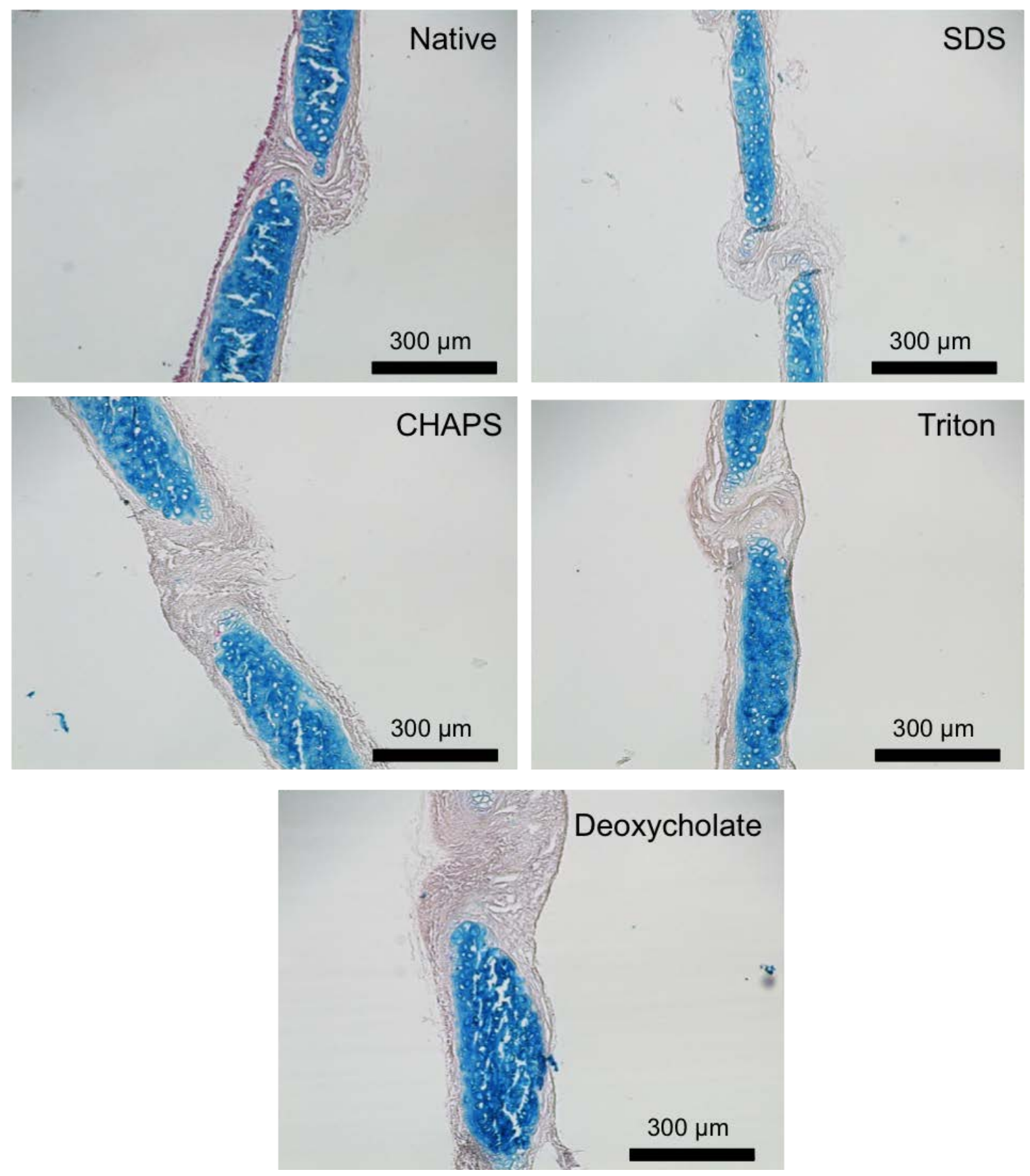

Figure 2.5: 10X Alcian blue stained detergent decellularized trachea scaffolds. Glycoaminoglycans are stained blue with nuclear material in purplish red. All grafts demonstrate maintenance of GAGs within intact cartilage rings. As seen in the CHAPS, SDS, Triton, and deoxycholate groups, nuclei and nuclear material are absent in all decellularized specimens 


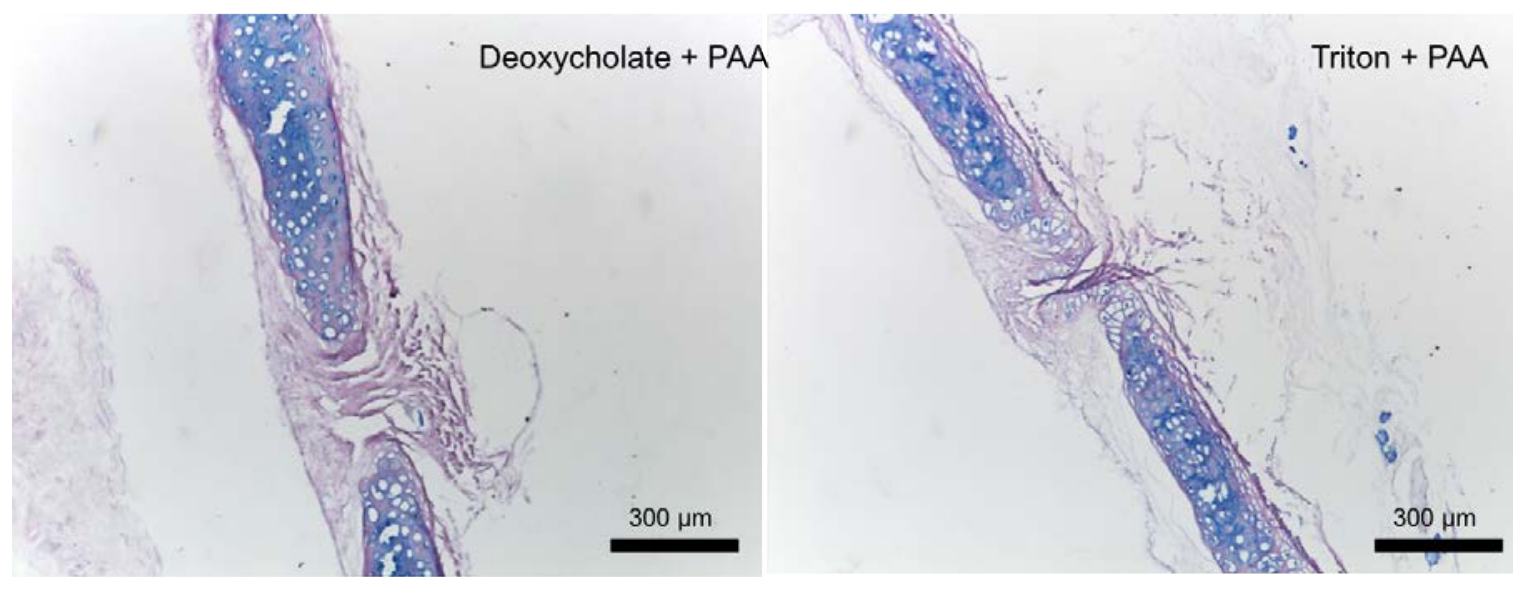

Figure 2.6: 10X Alcian blue stained PAA treated decellularized trachea. Grafts demonstrate retention of GAG within intact cartilage rings.

\subsubsection{Detergents Differentially Influence Collagen Microstructure and Matrix Associated Proteins}

A variety of detergents have been widely employed for decellularization of cartilaginous tissues, a number of these studies have shown histologic retention of native-like microstructure with both ionic and non-ionic detergents [194, 195]. However, few studies employ more in depth analysis techniques. It was originally planned that biochemical assays (Blyscan, etc.) would be employed to specifically measure ECM constituents. It was found commercially available assays for collagen, elastin, and GAGs were imprecise due to complex user input requirements. For example, one step requires manually blot drying residual fluid from an eppendorf tube, where excess fluid can dramatically effect the assay result. It addition, biochemical assays only measure removal, they are generally not capable of detection of disruption or denaturation. For these reasons, an alternate technique was sought. Differential scanning calorimetry (DSC) presented an excellent alternative, it is a sensitive technique whereby differences in the amount of enthalpy required to increase the temperature of a sample compared to a reference is measured as a function of temperature. DSC been used 


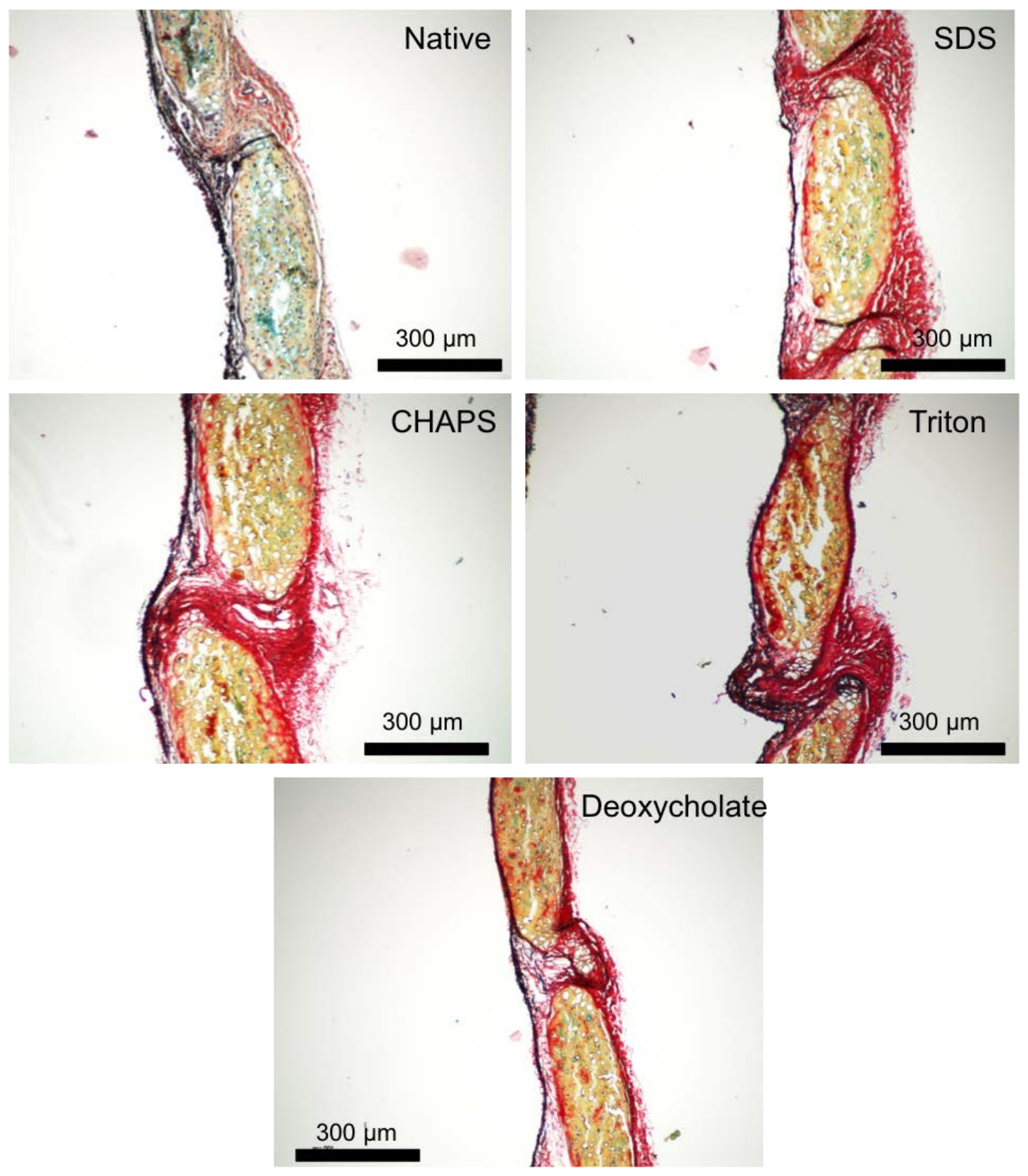

Figure 2.7: 10X Movat's pentachrome staining of non PAA treated, decellularized rat tracheas. Black: nuclei and elastic fibers, Yellow: collagen, Blue: mucin, Bright Red: fibrin, Red: Muscle. Partial removal or denaturation of basement membrane and elastic fibers is evident within ionic detergent groups (SDS and sodium deoxycholate) 


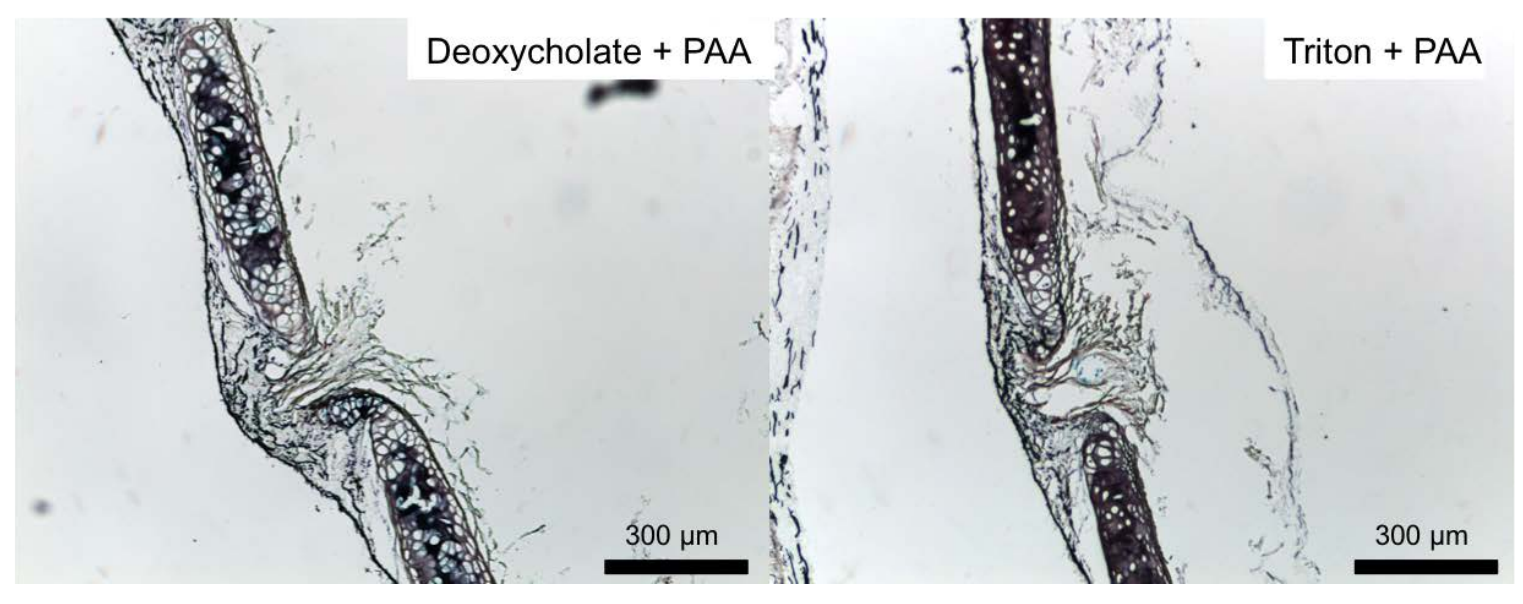

Figure 2.8: 10X Movat's pentachrome staining of PAA treated, decellularized rat tracheas. Black: nuclei and elastic fibers, Yellow: collagen, Blue: mucin, Bright Red: fibrin, Red: Muscle.

widely in the polymer and pharmaceutical industries, but is a relatively unused technique to investigate the thermal stability of proteins within an extracellular matrix scaffold. Various components within a scaffold have remarkably different denaturation temperatures, thus DSC can be used to not only assess compositional changes, but also, differences in protein cross linking, hydrogen bonding, and hydration states. Previously, to probe these questions detailed transmission electron microscopy (TEM) studies were necessary. As with any electron microscopy technique, TEM require extensive sample processing which may result in structural artifacts. The application of DSC to our tracheal scaffold enables us to assess micro-structural and compositional changes with each step of the decellularization process in a high throughput manner. In DSC, the main collagen peak appears at roughly $60^{\circ} \mathrm{C}$, with mature highly cross-linked fibers stable up to 75 or $80^{\circ} \mathrm{C}$. Matrix associated proteins including elastin and GAGs are represented as a broad shoulder from $35^{\circ} \mathrm{C}$ [196, 197]. Unsurprisingly, there are clear differences between detergent treatment groups. Most interestingly, results (Figure 2.9) suggest that SDS completely denatures the higher order collagen structures in both the fibrous and cartilaginous portion of the trachea. The shoulder above $70^{\circ} \mathrm{C}$ in the 


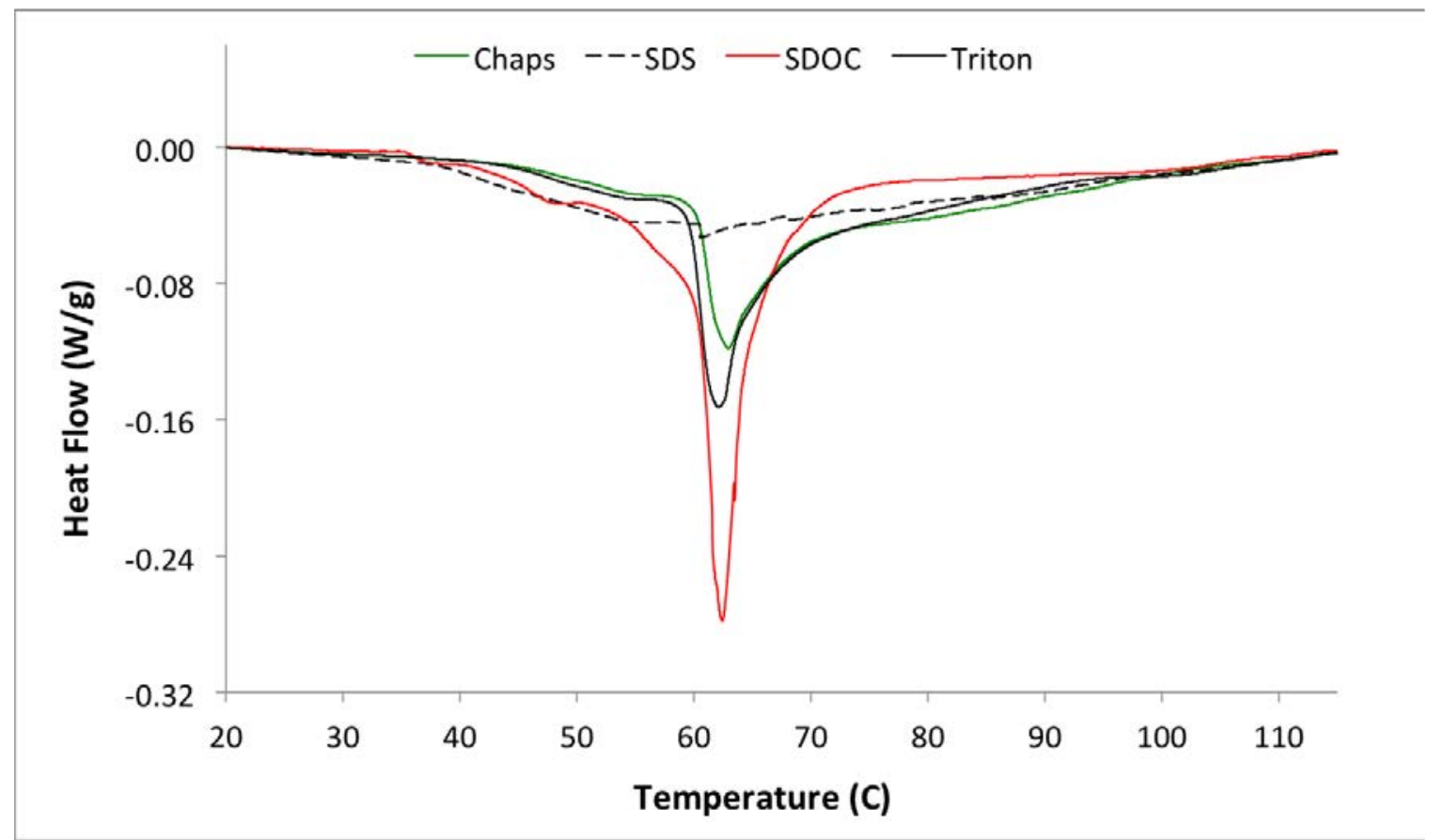

Figure 2.9: Differential scanning thermograph of non PAA treated decellularized rat tracheas. Primary collagen peak is located at $60^{\circ} \mathrm{C}$, with mature highly cross-linked fibers stable up to 75 or $80^{\circ} \mathrm{C}$. Matrix associated proteins including elastin and GAGs are represented as a broad shoulder from $35^{\circ} \mathrm{C}$ 
CHAPS and Triton X-100 indicates preservation of mature collagen cross-linking which is partially lost during deoxycholate treatment potentially explaining the resulting larger main collagen peak. We can also see that PAA treatment has a much more marked effect on the sodium deoxycholate group (Figures 2.9 and 2.10). Most commercially available PAA uses hydrogen peroxide as a stabilizer(up to $35 \% \mathrm{v} / \mathrm{v}$ ), which can result in artificial crosslinking of collagen structures, partially restoring the shoulder above $70^{\circ} \mathrm{C}$. Unpublished data has shown that increased PAA treatment times will eventually create a second distinct peak. In both PAA treatment groups, matrix associated proteins, and proteoglycans are denatured or removed, evidenced by the comparitive loss of the shoulder below $60^{\circ} \mathrm{C}$. These results are confirmed by histological analysis employing Herovici's stain, which differentially stains immature or uncrosslinked collagen. We can see from Figure 2.11 that SDS has a noticeably less intense mature collagen staining, while Triton X-100 and CHAPS maintain a much more native-like distribution and intensity of differential collagen staining.

Extracted data including, main peak temperature, and total enthalpy were assessed quantitatively. While shoulder onset temperature is a good measure of the retention of the less stable matrix bound proteins such as GAGs and laminin, statistical analysis found only a few significant differences between detergent groups, but broader trends are evident (Figure 2.12). Ionic detergents demonstrate a lower onset temperature and increased enthalpy (area under the curve) within this region. While seemingly counter-intuitive, it is possible that some of this effect may be driven by denaturation of collagen to a more gelatinous form (especially in SDS). For main peak temperature, the most notable finding is the lack of a SDS main collagen peak, which resulted in statistically significant differences between SDS and the remaining non PAA and PAA treated groups ( $\left.\mathrm{p}_{\mathrm{i}} 0.05\right)$. There were also significant differences observed between Triton, sodium deoxycholate, CHAPS and the two PAA treated groups. This finding is logical, given the conserved cartilage structure in Triton, deoxycholate and CHAPS, we would not expect a significant alteration in the thermal stability of fibrillar collagen within the scaffold. However, we would expect the total enthalpy to be significantly different for various processing parameters (Figure 2.13). Statistical differences were found between both PAA treated groups and all non PAA groups. Additionally, SDS was found to be significantly different that all of the non PAA treated specimens. Counter 


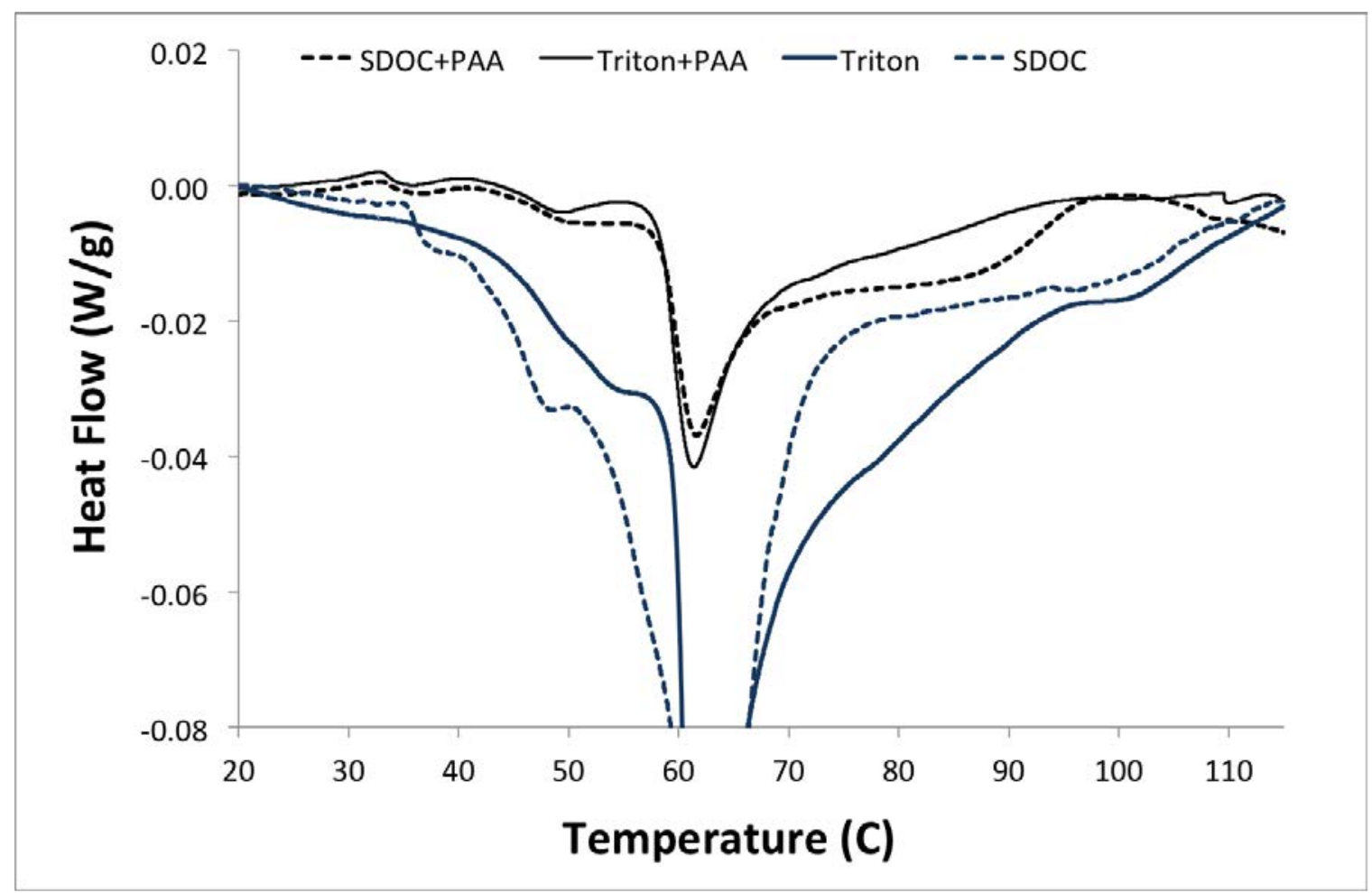

Figure 2.10: Differential scanning thermograph demonstrating comparison between PAA treated and untreated detergent decellularized rat tracheas. 

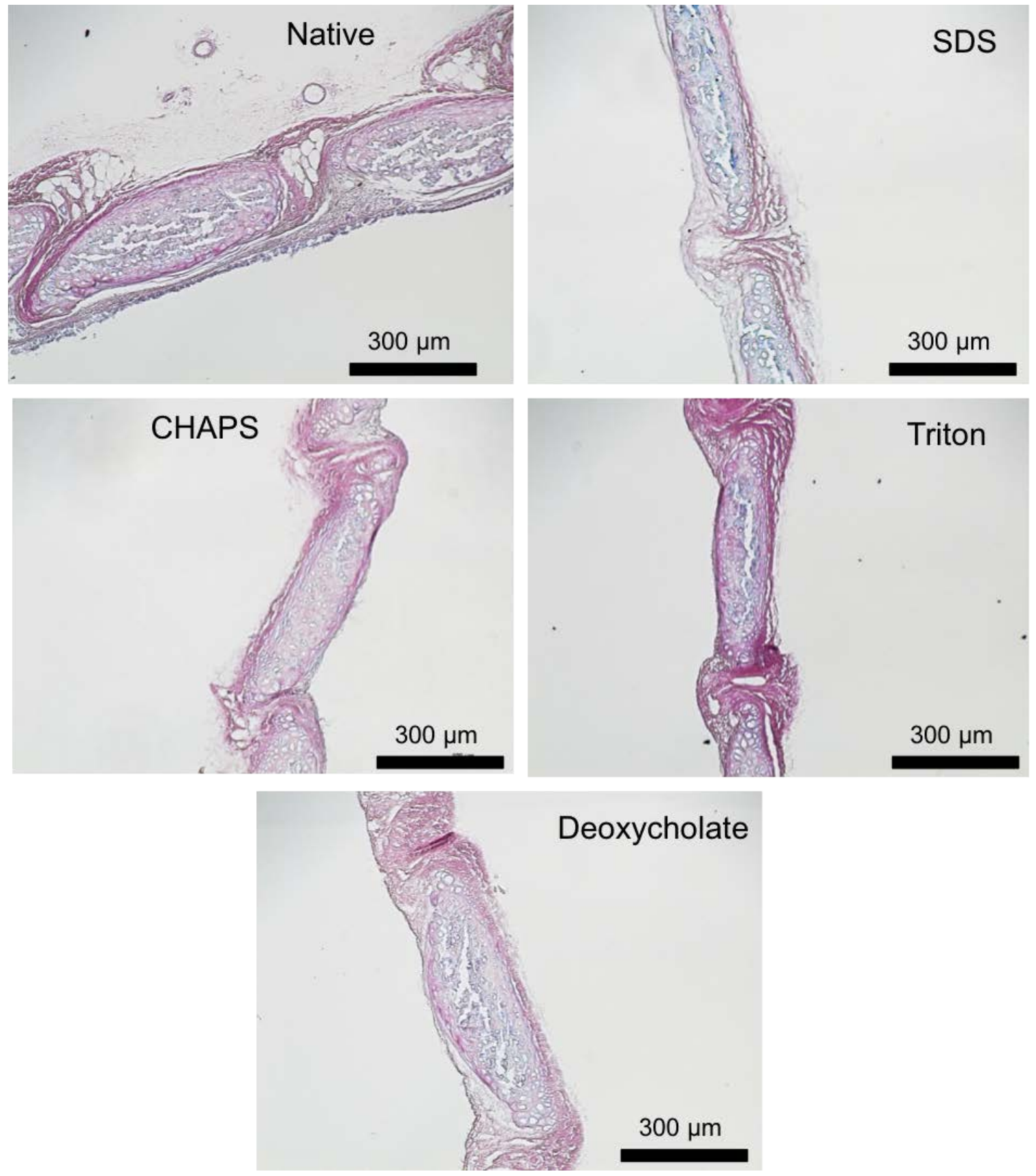

Figure 2.11: 10X Herovivi's staining of non PAA treated, decellularized rat tracheas, primarily esed to differentiate young and mature collagen. Herovici's stains young collagen and reticulum blue and mature collagen red while providing a yellow cytoplasm counterstain. Nuclei are stained blue to black. 
to our hypothesis, PAA seemed to have an strong, negative effect on the thermal stability of the ECM scaffolds as evidenced by the similarity between total enthalpy for PAA and SDS treated specimens. The hypothesized PAA cross-linking did not appear to globally increase tissue stability.

\subsubsection{Tracheal Mechanics}

Employing our existing apparatus, differences were not detected between decellularized groups or between acellular scaffolds and the native tissue. This was partly due to challenges associated with diameter detection and compounded by non-homogeneous deformation along the tracheal length. It is proposed that by employing a CCD camera to simultaneously image the entire length of the trachea instead of a single diameter some of these complexities may be overcome. Another potential approach could employ a pressure volume relation to compute an associated global "compliance" measure. This is a well established approach in cardiovascular biomechanics, especially for larger vessels and the myocardium[198]. However, unlike the vasculature, the complex geometry and lack of circular symmetries make this approach more mathematically challenging. Another, simpler, approach could avoid quantifying the pressure diameter relationship of decellularized trachea and instead measure the collapse and burst pressures, which are likely more predictive of poor in vivo performance. Another suggested improvement is to incorporate suture retention testing prior to implant to avoid a predictable graft failure mechanism.

\subsubsection{Limitations and Future Work}

Due to the extraordinarily complex biological milieu, mechanistic studies optimizing a specific decellularization procedure are not yet possible. Not only is the complexity of native extracellular matrix not fully understood, but work is still ongoing to better characterize the decellularization process itself. With our current level of understanding empirical studies are necessary, but care must be taken to avoid needless experimentation. The tracheal tissue engineering literature is filled with claims of success and failure, but little is learned from each of these experiments, since often studies assess only a single empirically derived 

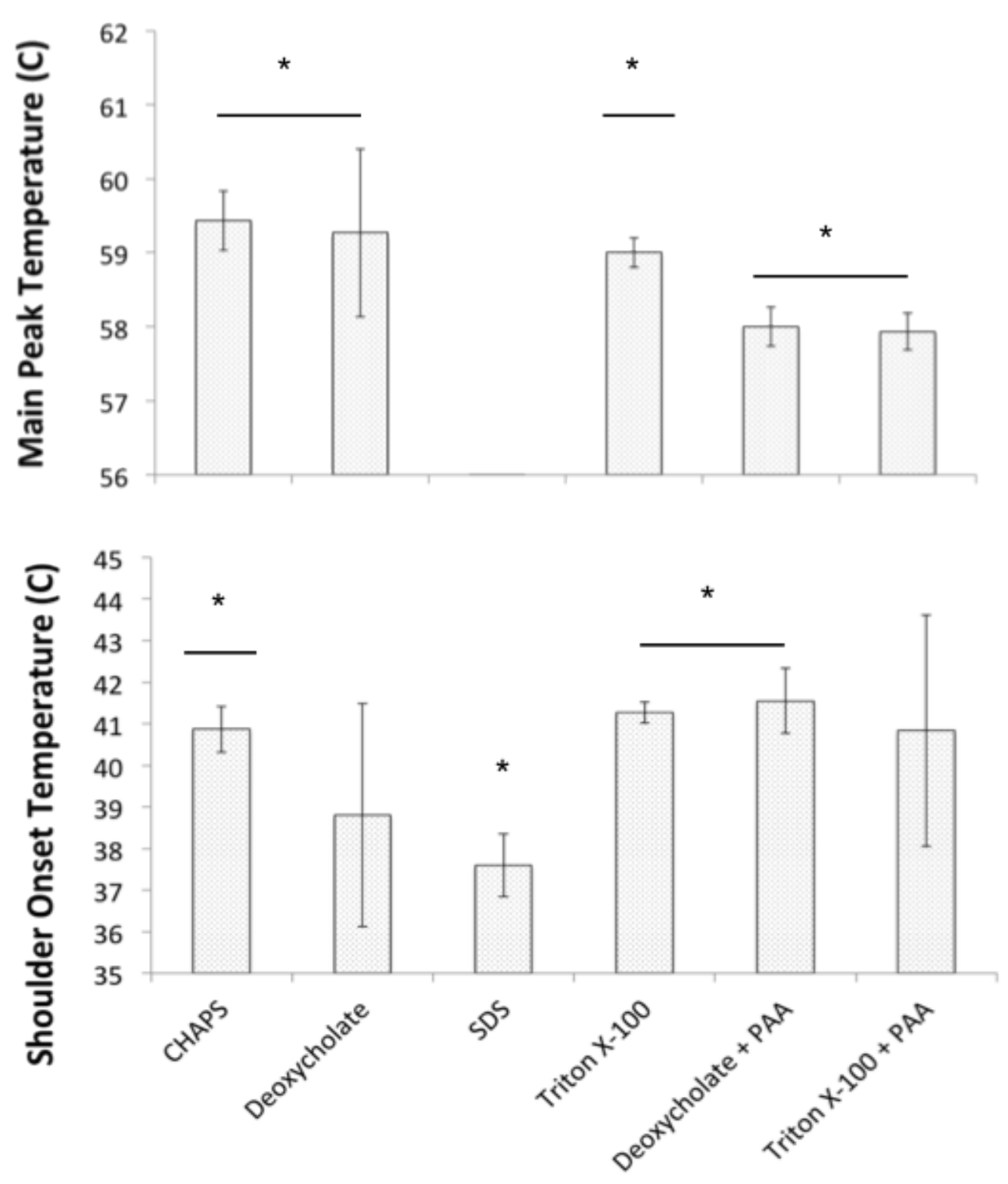

Figure 2.12: Main collagen peak and shoulder onset temperature computed from DSC thermogram, presented as mean plus/minus standard deviation (minimum $\mathrm{n}=3$ for each group). Top: Main peak temperature representing the maximal value for the largest peak, computed as the global minimum of the zero first derivative, SDS did not have a clear peak and was therefore, excluded. Bottom: Shoulder onset temperature for the main peak. Computed as the temperature at which a 5 degree moving average first deviated from a linear baseline. 


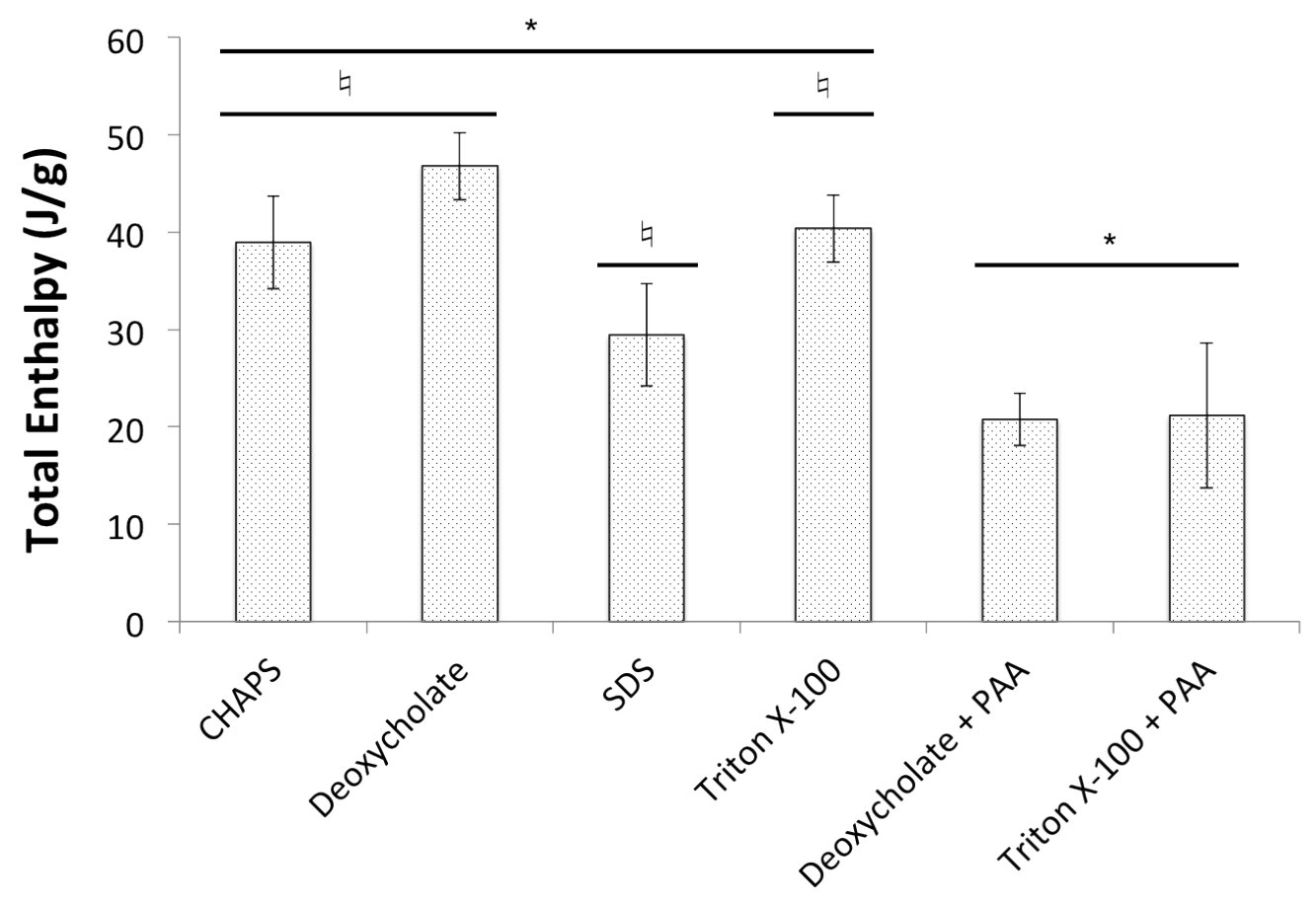

Figure 2.13: Total enthalpy, computed from DSC thermogram, presented as mean plus/minus standard deviation (minimum $\mathrm{n}=3$ for all groups). Computed as the total area between the curve and a fitted linear baseline. Statistical significance was calculated with a MANOVA and post hoc Tukey's test, significant differences were found between all detergent only specimens and both PAA treated groups. A significant difference was also found between SDS and the remaining detergent only specimens. 
scaffold [153, 199, 200]. Clearly we cannot claim to have developed a comprehensive design space, but this work definitely represents an incremental improvement in the understanding of tracheal decellularization.

Compounding this confusion in the literature is the lack of currently established metrics to characterize successful decellularization of the trachea. General guidelines for decellularization as a whole simply suggest complete removal of cellular nuclei, reduction of DNA to less than $5 \mathrm{ng} / \mathrm{mg}$ of tissue with lengths less than 200 base pairs [201]. Further compositional characterization of tracheal scaffolds could be enormously beneficial to predict and interpret in vivo findings. Immunofluorescence, visualizing basement membrane constituents might also be highly predictive of in vivo epithelial recolonization. Further, proteomics or western blotting to assess remnant proteins, either cellular or matrix bound might predict potential immune response and shed light on signaling cascades involved in remodeling. Finally, functional assays may be employed to assess the biological activity of specific proteins of interest.

\subsection{CONCLUSIONS}

Patients diagnosed with primary tracheal cancers were historically palliated in the absence of any effective treatment options. Recent preliminary clinical studies have shown ECM scaffolds provide a viable option for patients who previously had none [148]. This approach utilizes decellularized human tracheas prepared via a detergent enzymatic method with sodium deoxycholate. In addition to dire supply limitations, sodium deoxycholate is harsh ionic detergent known to denature proteins both in vitro and in vivo [180, 181, 182, 183, 184]. More than simply removing cellular antigens and debris, ECM grafts processed with sodium deoxycholate may not retain the growth factors, proteoglycans, and other matrix proteins in a native configuration capable of providing cues to infiltrating host cells. Therefore, we have tested alternative approaches and demonstrated that an abbreviated detergent enzymatic method possible may be successfully employed in a relevant mammalian model. As hypothesized, alternative, less harsh decellularization agents were equally capable of producing 
an acellular trachea ECM. However, there were clear differences in tracheal microstructure and composition. Interestingly, our hypothesis that PAA would have a minimal effect on ECM structure was also shown to be incorrect. While the gross and histologic appearance of PAA treated specimens is nearly identical to untreated specimens, DSC demonstrated a significant impact on graft structure and composition, further necessitating the use of more sensitive analysis techniques. The observed differences described in this aim will be vital in specific aim 2, when these grafts will be implanted in vivo and salient ECM features will be correlated with scaffold remodeling and fate.

\subsection{ACKNOWLEDGEMENTS}

We would like to thank Dr. Wendell Sun for his assistance with DSC and Nathanael C. Yoder for the use of his invaluable peak finder code. 


\subsection{SUCCESSFUL ORTHOTOPIC TRANSPLANTATION OF DECELLULARIZED RAT TRACHEA}

\subsection{INTRODUCTION}

In the previous chapter it was observed that even simple variations to a single decellularization procedure can have significant effects on scaffold properties. These properties likely drive host response and dictate implant fate. Employing an in vivo rodent model allows us to couple similarities in mammalian airway structure and biology with the relative ease of a small animal model. Additionally, a rodent model allows future mechanistic studies utilizing mutant strains to investigate specific relevant signaling cascades. To date, many of the preclinical studies evaluating tracheal transplants have been heterotopic, typically implanted in a sub-dermal position [200, 199, 202]. Heterotopic grafts are useful for verification of biological and immunologic compatibility, however, their predictive ability for orthotopic implantation is limited. Other studies have employed orthotopic implantation, but have tested only a single experimental group, often controlling for in vitro cell seeding rather than processing conditions $[151,152,153]$. Of those, many were window patches which have increased survival compared to full circumference grafts, but fail to address the underlying

clinical need. To avoid this myriad of concerns, we propose to employ an orthotopic rat transplantation model to test the several group of decellularized tracheas characterized in specific aim 1. Given the measured scaffold degredation and denaturation data is specific aim one we hypothesize that ionic detergent use and PAA treatment will be highly correlated with degraded performance in vivo. To specifically test this claim, full circumference grafts will be implanted for 12 weeks in vivo. Upon explant, the tissue will be thoroughly characterized, taking special care to evaluate cellular infiltration, epithelial differentiation, and site specific function. 


\subsection{MATERIALS \& METHODS}

\subsubsection{Implant}

Lewis rats were anesthetized with intraperitoneal injections of ketamine hydrochloride (100 $\mathrm{mg} / \mathrm{kg}$ ) and xylazine hydrochloride $(10 \mathrm{mg} / \mathrm{kg})$. Animals were placed in a supine position with extremities secured. The surgical site was treated with betadine and aseptic surgical technique was utilized. Intraoperative body temperature was maintained with a heating pad. A midline cervical incision was created with visualization through a surgical microscope. The graft was prepared by removing fluid from the lumen and any extraneous tissue. Proximal distal orientation was maintained. The cervical esophagus and other vascular structures were sharply dissected from the trachea with care not to damage the recurrent laryngeal nerves. The trachea was transected three or four rings distal to the epiglottis and two rings were removed. Meticulous hemostasis was performed to limit fluid entering the airway, and signs of respiration were continually monitored. The animals were able to maintain spontaneous ventilation through the tracheostomy. A 5-ring donor tracheal graft was transplanted with end-to-end anastomoses starting distally with care to align the membranous portion of the tracheal graft with the membranous portion of the recipient trachea. The cartilaginous portions were secured with interrupted 7-0 Prolene sutures, two near the dorsal ends of the cartilage rings and two placed on the ventral aspect of the tracheal repair as seen in Figure 3.1. The strap muscles were approximated and the skin was closed with 5-0 PDS suture. Surgery averaged approximately 20 minutes.

\subsubsection{Post-operative Care}

After surgery, animals were ear punched for identification and allowed to fully recover from anesthesia. Rats were individually housed in standard cages. Food and water was supplied ad libitum. The following medications were administered as subcutaneous injections for five days following surgery: buprenorphine $(0.05 \mathrm{mg} / \mathrm{kg})$ twice daily for pain relief, gentamicin $(8 \mathrm{mg} / \mathrm{kg}$ ) once daily for infection prophylaxis. In the days following implant procedure the animals were monitored daily for infection, inflammation, or delayed healing of the site. 

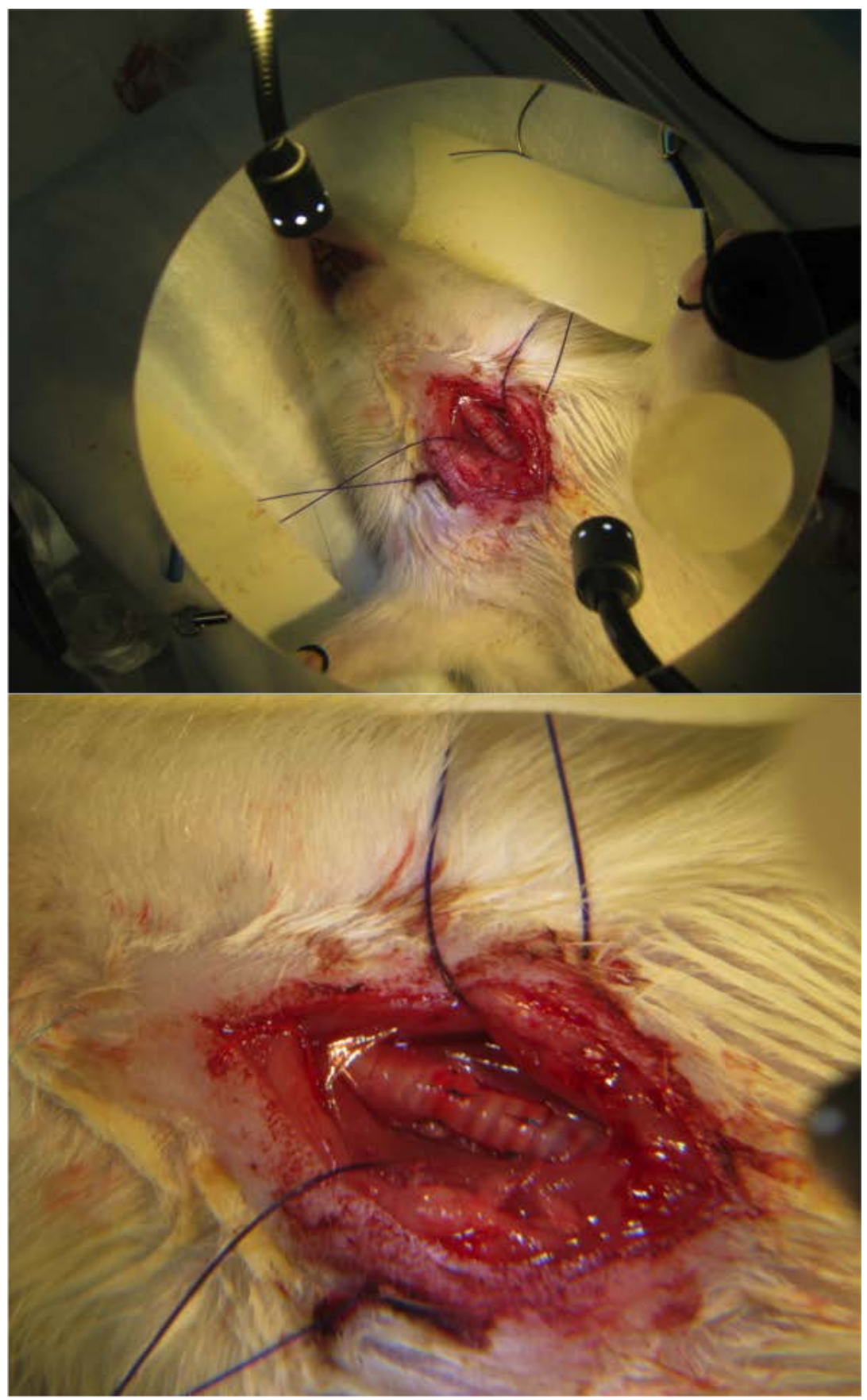

Figure 3.1: Intraoperative images of orthoptopic transplantion. Top: Decellularized trachea after graft reanastomosis prior to strap muscle reapproximation and closure. Bottom: Zoomed image demonstrating careful separation of the neurovascular bundle and graft alignment 
Special care was taken to monitor for any apparent respiratory distress, pain, or general discomfort. Visual monitoring of food intake was made daily. After 14 days, animals were socially housed.

\subsubsection{Explant and Tissue Processing}

Twelve weeks after surgery, rats were anesthetized with intraperitoneal injections of ketamine hydrochloride $(100 \mathrm{mg} / \mathrm{kg})$ and xylazine hydrochloride $(10 \mathrm{mg} / \mathrm{kg})$. An incision was made in the abdominal cavity and the abdominal aorta was transected to exsanguinate the animal. The entire length of the trachea was exposed via sternotomy with concomitant mid-line cervical incision. The trachea from the larynx to just proximal to the bifurcation were excised with the use of a surgical microscope. Grafts were mildly flushed with and stored in cold Leibovitz's L-15 medium (L15) supplemented with 10\% FBS for immediate ciliary beat analysis with differential interference contrast microscopy.

\subsubsection{Ciliary Beat Frequency}

Each trachea was trimmed of extraneous tissue under a dissecting microscope. Gross histological images were captured during dissection. Trachea were then cut longitudinally, half was fixed for scanning electron microscopy while the other half was secured luminal side down on a 35-mm glass-bottomed culture dish (Willco Wells) using a glass coverslip covered with a silicone sheet containing a small window to form a chamber (Figure 3.2). Cilia dynamics were captured at room temperature with a 100X differential interference contrast (DIC) oil objective and a Leica inverted microscope (Leica DMIRE2). Movies were made with a Phantom v4.2 camera (Vision Research) at 200 frames/s (fps). To quantify ciliary beat frequency $(\mathrm{CBF})$, ImageJ was used to examine cyclic variations in pixel intensities corresponding to ciliary strokes (Figure 3.2). Specifically, at least 400 frames of cilia motion were imported into ImageJ and a line was manually drawn through the beating cilia. The video was then re-sliced along the line by time, resulting in a plot with pixel intensity plotted along the $\mathrm{x}$ axis for each video frame. $\mathrm{CBF}$ was then computed manually by measuring cyclic pixel variation over time. For each specimen more than three randomly selected areas were 
imaged from each trachea in order to calculate mean CBF for each treatment group. After analysis, specimens were removed from the silicone chamber and fixed in neutral buffered formalin for histological analysis.

\subsubsection{Scanning Electron Microscopy}

Tissue was fixed in 2.5\% glutaraldehyde in $0.1 \mathrm{M}$ PBS ( $\mathrm{pH} 7.4$ ) for 60 minutes. It was then thoroughly rinsed in 3 changes of $0.1 \mathrm{M}$ PBS for 15 minutes each. Tissue was then fixed in $1 \%$ osmium tetraoxide in $0.1 \mathrm{M}$ PBS for 60 minutes followed by 3 rinses in $0.1 \mathrm{M}$ PBS for 15

minutes each. Specimens were then dehydrated in graded series of alcohol (in PBS) for 15 minutes each: 30, 50, 70, and 90\% ethanol followed by 3 rinses in 100\% ethanol. Specimens were then mounted on studs with silver paste and sputter coated with palladium/gold and stored in a dessicator until imaging. Images were obtained at several magnifications to visualize epithelial coverage, ciliation, and lumen topology.

\subsubsection{Histology}

H\&E and Movat's Pentachrome staining was performed on explant tissue using standard paraffin histological techniques.

\subsubsection{Statistics}

For survival curves, a log-rank test was employed to evaluate statistical significance. Statistical comparisons between cilia function was accomplished via one-way ANOVA analysis with bonferroni correction. Significance was gained for all tests at $\mathrm{p}<0.05$. 

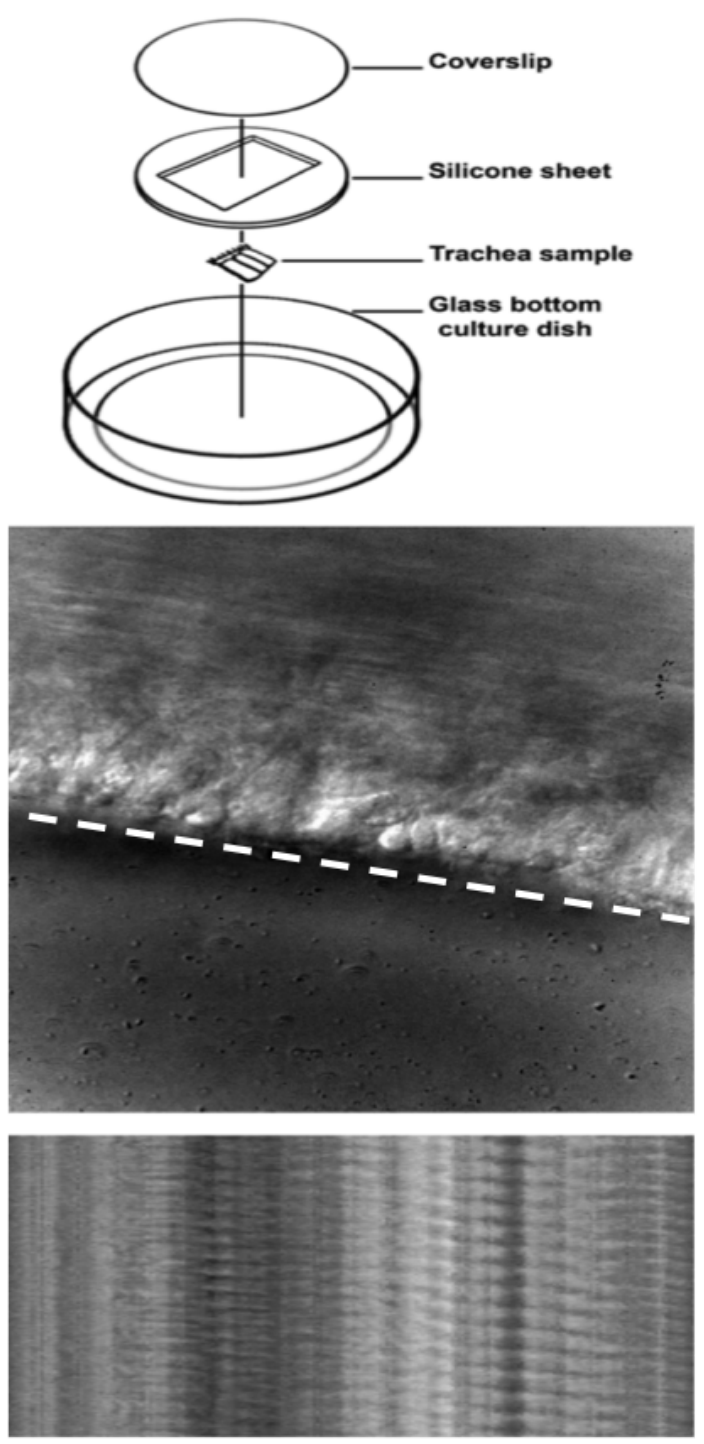

Figure 3.2: Schematic of ciliary beat frequency analysis. Top: The freshly explanted and transected trachea is sandwiched between a glass coverslip and glass culture well, silicone sheeting is used as a spacer. Middle: DIC microscopy is used to capture at least 400 frames of cilia motion. The video is imported into ImageJ and a line is manually drawn through the beating cilia. Bottom: The video is re-sliced along the line by time, resulting in a plot with pixel intensity plotted along the $\mathrm{x}$ axis for each video frame. Ciliary beat frequency is computed manually by measuring cyclic pixel variation over time 


\subsection{RESULTS AND DISCUSSION}

\subsubsection{Animal Survival Varies with Treatment, PAA Correlated with Positive Outcomes}

In general, animals tolerated surgery well and recovered quickly, resuming normal activity levels within 12 hours. All deaths, except one, occurred within 10 days of surgery. Results demonstrate increased survival trends (non significant) of Triton X-100 and SDS groups compared to deoxycholate. Oddly, CHAPS decellularized grafts performed significantly worse (Figure 3.3) than any of the other 3 non PAA treated detergent groups. There were no deaths in the PAA treatment groups, demonstrating similar performance to the best untreated grafts (Figure 3.4). More interestingly, the simple addition of a PAA rinse significantly improves survivial of deoxycholate recipient animals at the 12 week time point.

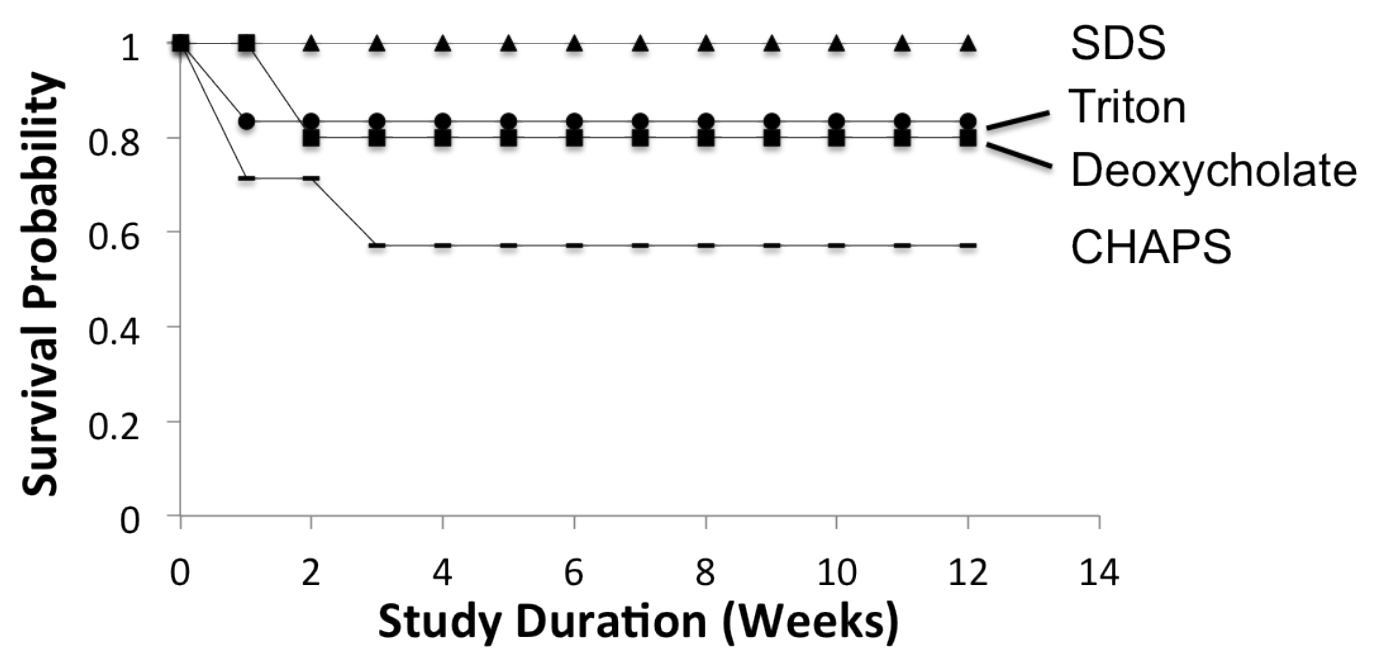

Figure 3.3: Post-operative survival curves demonstrating increased survival among SDS and Triton X-100 experimental groups. 


\subsubsection{Mortality Driven by Obstruction}

The observed deaths were likely not due to immediate surgical complications such as lumen misalignment or inadequate anastomosis since necropsy of Triton, CHAPS, and sodium deoxycholate grafts demonstrate excellent anastomosis, however, there was substantial mucous observed within the grafted sections. Therefore, it was concluded that mucuous obstruction was the most probable cause of death (Figures 3.5). Interestingly, neither stenosis nor malacia was observed in any of the early deaths. Given the invasive nature of the procedure excess mucuous production is probably unavoidable with any graft, however, it is possible that many, if not all, of the early mortalities would be avoidable in a clinical setting. For example, it is known that patients who received tissue engineered transplants required significant post-operative care including temporary stenting and endoscopic mucuous removal [203]. Increased mucous production is also present during bacterial or fungal infection, but this possibility was eliminated due to lack of symptoms and normal histological analysis. The natural next supposition is that increased mucous production is correlated with a stronger acute inflammatory response, which would suggest that CHAPS specimens have poorer in vivo cytocompatibility. This directly contradicts the results we initially predicted given in vitro ECM analysis and implies that we cannot simply employ similarity to native tissue as the metric for sucessfull decellularization. This is further supported by the lack of deaths within the PAA treatment groups. The combination of these non-intutitive findings oppose traditional wisdom in the tissue engineering field, which generally suggests that during decellularization the less a tissue is disturbed, the more likely it is to result in positive and physiologic remodeling. Other reports have confirmed these findings, for example, Carruthers demonstrated poor in vitro compatibility of several tissue types after CHAPS decellularization [204]. They suggests these results are, in part, due to loss of ECM constituents and denaturation of the collagen fiber network. That explanation is based on limited histological analysis and directly contradicts our more precise DSC findings from chapter 2. Instead, we believe that the poor survivial is more likely due to inadequate removal of the detergent or remnant cellular proteins. However, due to the early nature and 
small number of these deaths, the reepithelialization and cellular differentiation of animals surviving the full 12 weeks is likely substantially more indicative of overall host reponse.

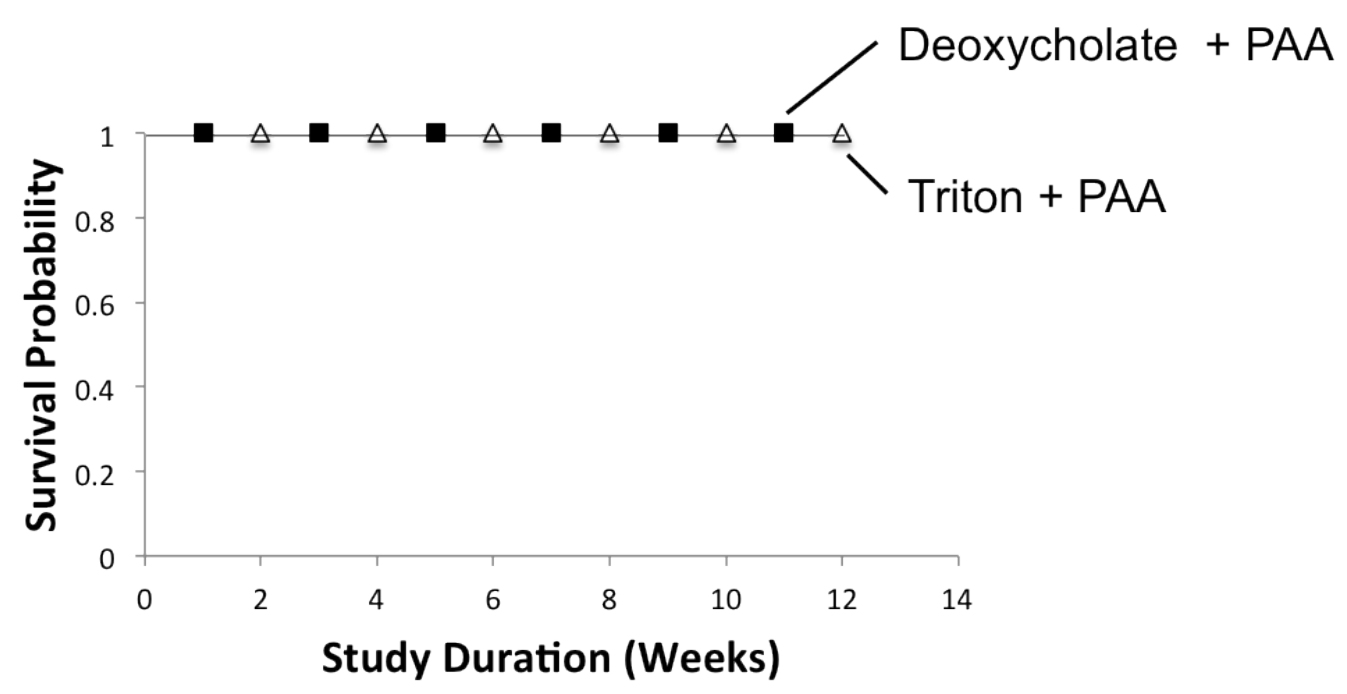

Figure 3.4: Post-operative survival curves showing $100 \%$ survival among both PAA posttreated experimental groups.

\subsubsection{Processing Conditions Predetermines Host Mediated Remodeling}

All animals surviving to study end had patent grafts with intact and mechanically sound cartilage. The tissue was elastic in the longitudinal direction, while appearing to maintain native like circumferential stiffness. When dissected for ciliary beat frequency analysis the graft lumens appeared to have been fully reepithelialized, however, stenosis was readily apparent in many of the CHAPS and sodium deoxycholate specimens (Figure 3.6). Interestingly, SDS explants did not demonstrate stenosis, but fibrotic strictures were observed luminally between the cartilage rings. All non-PAA treated grafts demonstrated a yellow hue, potentially suggesting the onset of necrosis. Visible neovasculature similar to the flanking native segments was not observed in the CHAPS, SDS, or Triton X-100 groups. Treatment with PAA seems to dramatically improve the gross morphology effectively decreasing stricture 

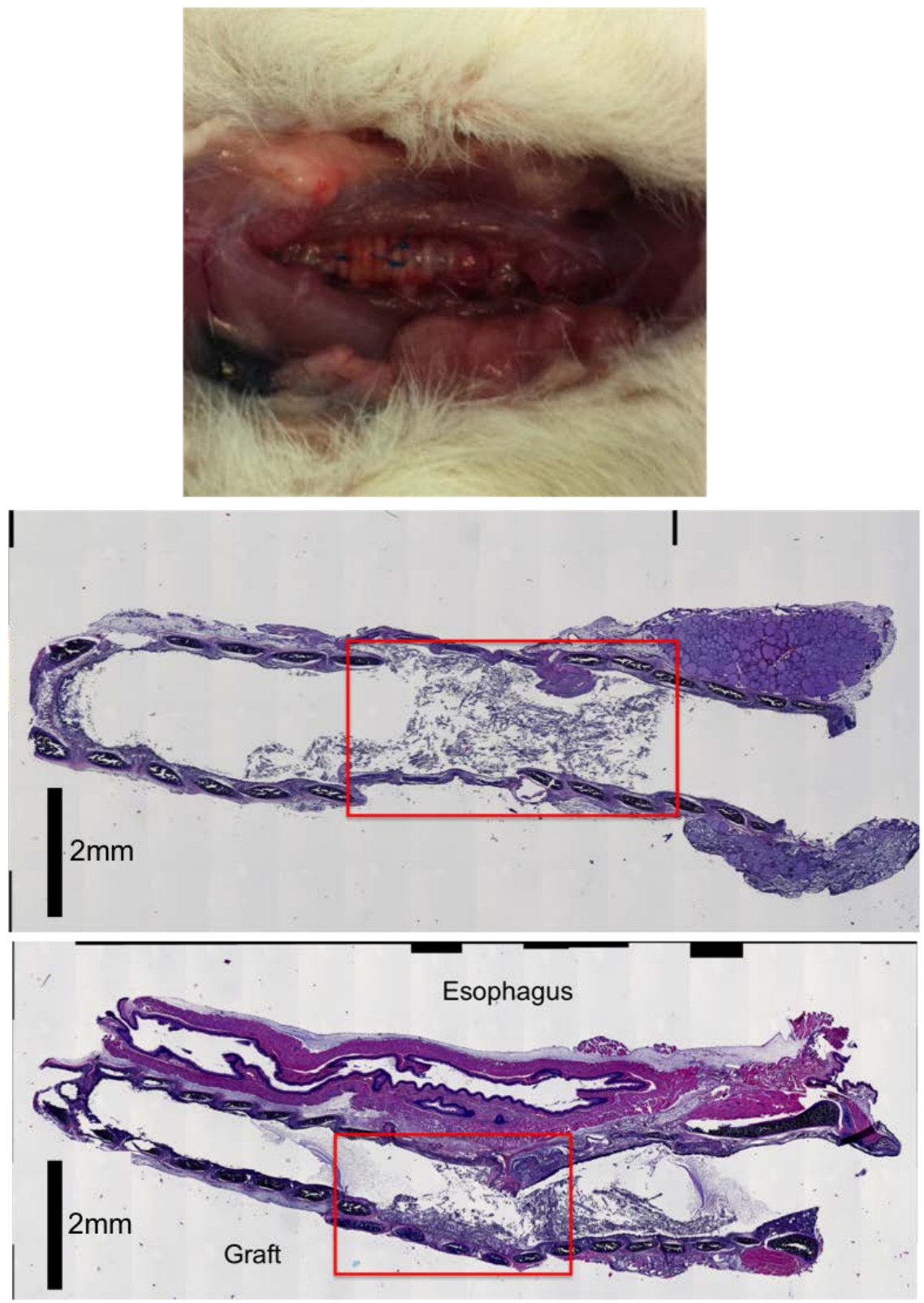

Figure 3.5: Top: Gross appearance of CHAPS graft at necropsy. Middle CHAPS necropsy H\&E section demonstrating mucous obstruction in red, Bottom: Triton necropsy also demonstrating lack of stenosis and muscous obstruction in red. 
formation and stenosis, which we believe are metrics predictive of longer term in vivo performance. PAA treated grafts also demonstrate a healthy, native appearance with distinct vasculature. These findings in combination with the mortality data further contradict our initial hypotheses. Overall, it appears that PAA is strongly correlated with revascularization and tissue integration, while CHAPS treatment is highly predictive of poor performance. Most interestingly, PAA seems to "rescue" the previously lackluster sodium deoxycholate specimens, as measured by mortality and gross appearance.

From the review of clinical and experimental literature, we believe that the lack of revascularization is the largest single hurdle for tracheal reconstruction and replacement. Many earlier studies demonstrated that insufficient revascularization was a major obstacle to successful remodeling in living tissue transplantation. Further attempts demonstrated vascularized grafts are much less likely to necrose than free patches $[90,91,92,93,10]$. It is likely that the necrosis resulting from non-vascularized cells causes a chronic inflammatory response driving the negative surgical outcomes in these historic studies. One significant benefit of decellularized tissue is that the slow colonization of the graft lumen with metabolically active epithelial cells occurs in parallel with the restoration of vascular supply. Immediately at implant the decellularized graft is simply a structural element and does not require significant vascularity to support it's function. Thus, we believe that the demonstrable improvement in PAA treated specimens is largely due to improved revascularization. While it is not necessarily possible to decouple revascularization from broader in vivo cytocompatibility, distinguishing the timeline of the epithelial and vascular regeneration may be beneficial to establish a preliminary causal structure. This structure would be best investigated through detailed histological anlyses at study mid points, preferably at 4 and 8 weeks.

Unfortunately, another limitation of the current study is that we are unable to specifically determine the mechanisms underpinning these findings, but will instead endeavor to make educated guesses given prior ECM characterization and the existing body of literature. For example, it is known that upon ECM implantation, host macrophages begin immediately infiltrating the scaffold. Recent studies have shown that these macrophages are potent modulators of disease and tissue remodeling following injury. M1 and M2 macrophages have also been shown to play distinct roles in tissue remodeling following injury and differential 
macrophage phenotype may catalyze either positive or negative tissue remodeling. Effectively, the biochemical and structural characteristics of the implanted device influence the host macrophages, altering the polarization profile and effectively predermining the host response. Differential macrophage staining may be employed in future studies to further investigate the host immune response [205, 206, 207]. Non-polar membrane bound proteins are involved with cell to cell recognition and are most likely to influence macrophage polarization and thus drive a negative immune response. Since CHAPS is the weakest detergent with the smallest micelle it may simply be incapable of properly solubilizing and removing many remnant membrane bound proteins. This inability to remove non-polar membrane bound proteins may have contributed to the lack of revascularization, eventually leading to stenosis and necrosis. Given the weaker monomer affinity and low molecular weight micelles we can most likely eliminate insufficient removal of CHAPS as a significant factor.

When considering PAA, there are several potential mechanisms which may be responsible for the notable improvement in longer term grafts. First, PAA has been shown in some studies to aid in removal of remnant detergent and enzymes [208], which may substantially improve the host cellular response. This would potentially explain the improvement in sodium deoxycholate and Triton X-100 groups, but unfortunately, since we did not test CHAPS and SDS with a PAA rinse we cannot specifically confirm this supposition. Second, PAA treatment has been shown to increase porosity in a number of dense collagenous tissues, including the tendon [209], which could improve cellular infiltration and subsequently increase angiogenesis. This supposition could be readily examined by the mid point analyses proposed earlier to assess rate of revascularization. It is also possible that PAA may have acted to partially eliminate endotoxins that may have resulted from bacterial growth during processing. It has been suggested that endotoxin present within the ECM may also affect signalling through the TLR4 pathway, resulting in a pro-inflammatory response [210]. To test this hypothesis, we could simply assess endotoxin levels before and after the PAA processing step. 
Necrosis
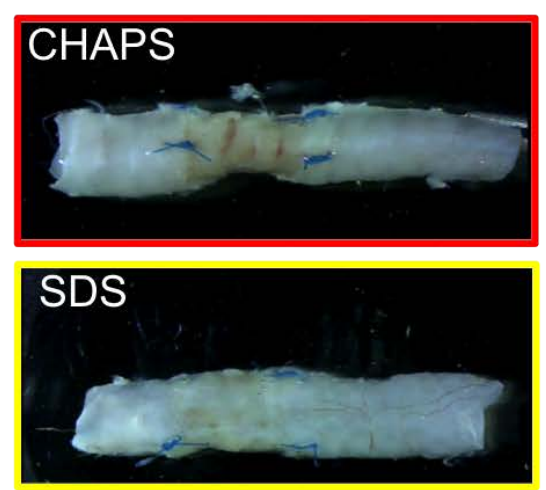

\section{Triton}

\section{Angiogenesis}
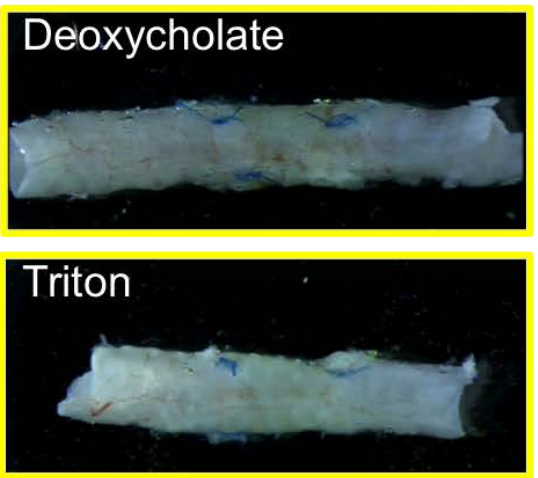

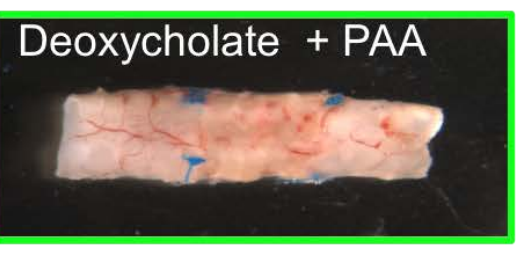

\section{Triton + PAA}

Figure 3.6: Gross morphology of representative explanted tracheal grafts. Significant stenosis in CHAPS and sodium deoxycholate groups. All non-PAA treated grafts demonstrated a yellow hue, potentially suggesting the onset of necrosis. Neovasculature is not readily apparent in the CHAPS, SDS, or Triton X-100 groups. Treatment with PAA dramatically improves graft appearance at explant. PAA treated grafts demonstrate a healthy, native appearance with distinct vasculature 


\subsubsection{Decellularized Grafts Support Epithelial Growth and Differentiation}

As discussed above, all grafts were fully epithelialized by 12 weeks, however, there were significant differences in the level of appropriate cellular differentiation between treatment groups. This is to be expected given the extraordinary differences observed in the gross morphology. Unsurprisingly, reepithelialization mirrored gross observational findings in all the experimental groups. CHAPS explants were uniformly coated in squamous epithelium (Figure 3.8), while SDS specimens showed mostly squamous coverage with localized patches of ciliated epithelium (Figure 3.9). Among the non PAA treated grafts, only Triton X-100 demonstrated any significant and uniform epithelial differentiation with cilia development. As we can see from Figures 3.10 and 3.11, PAA treated groups demonstrated excellent ciliation compared to their untreated counterparts, with the most pronounced difference between the sodium deoxycholate groups. It was hoped that immunofluorescent (IF) and histological analysis would be possible to probe the relative prevalence of ciliated epithelium and goblet mucosal cells to better establish the presence of all lineages of differentiated epithelium. However, once it became clear that the functional analysis effectively prevented further histological analysis it was determined that observing and quantifying ciliary beat frequency was more vital to overall study goals.

It is entirely possible that alteration or removal of basement membrane constituents as a result of decellularization disrupts the mechanical and biological cues that assist with site specific differentiation. However, the anomalous improvement in sodium deoxycholate and Triton X-100 after PAA treatment would make this argument less compelling. Instead it may be better explained by removal of residual detergent. Since sodium deoxycholate has a much higher monomer affinity to proteins, it might also explain the differential improvement between Triton X-100 and sodium deoxycholate. Our proposition that increases in porosity leading to improved vascularity may also have a synergistic effect. This would explain the sparser ciliation and decreased cilia function in non PAA treated specimens. 

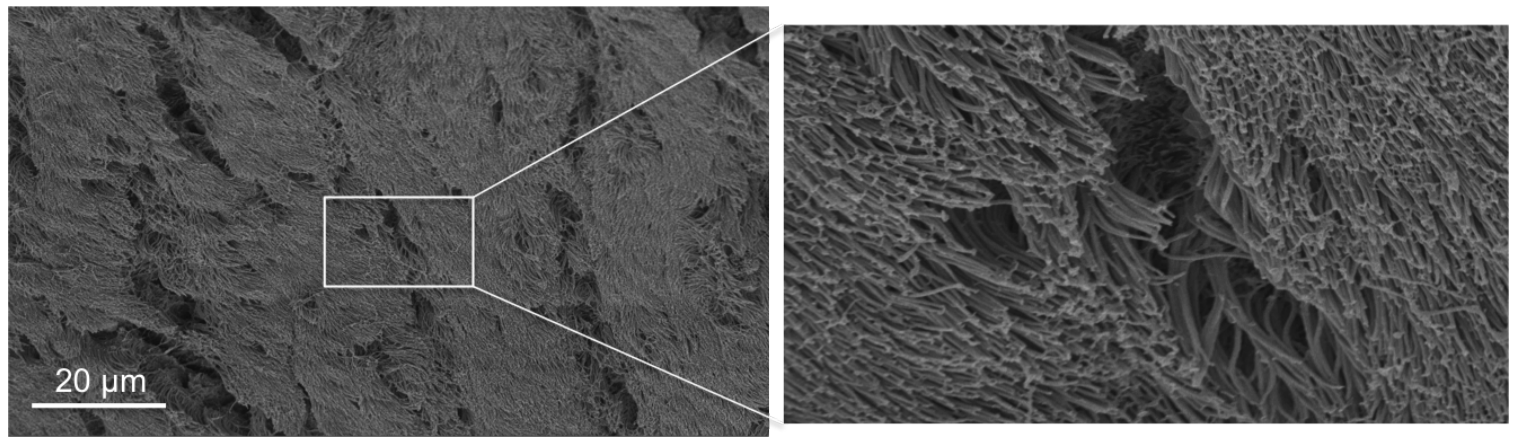

Figure 3.7: Left: Scanning electron micrograph (1000X) of the native rat trachea. Right: Expanded view (5000X) of dense mature ciliated epithelium
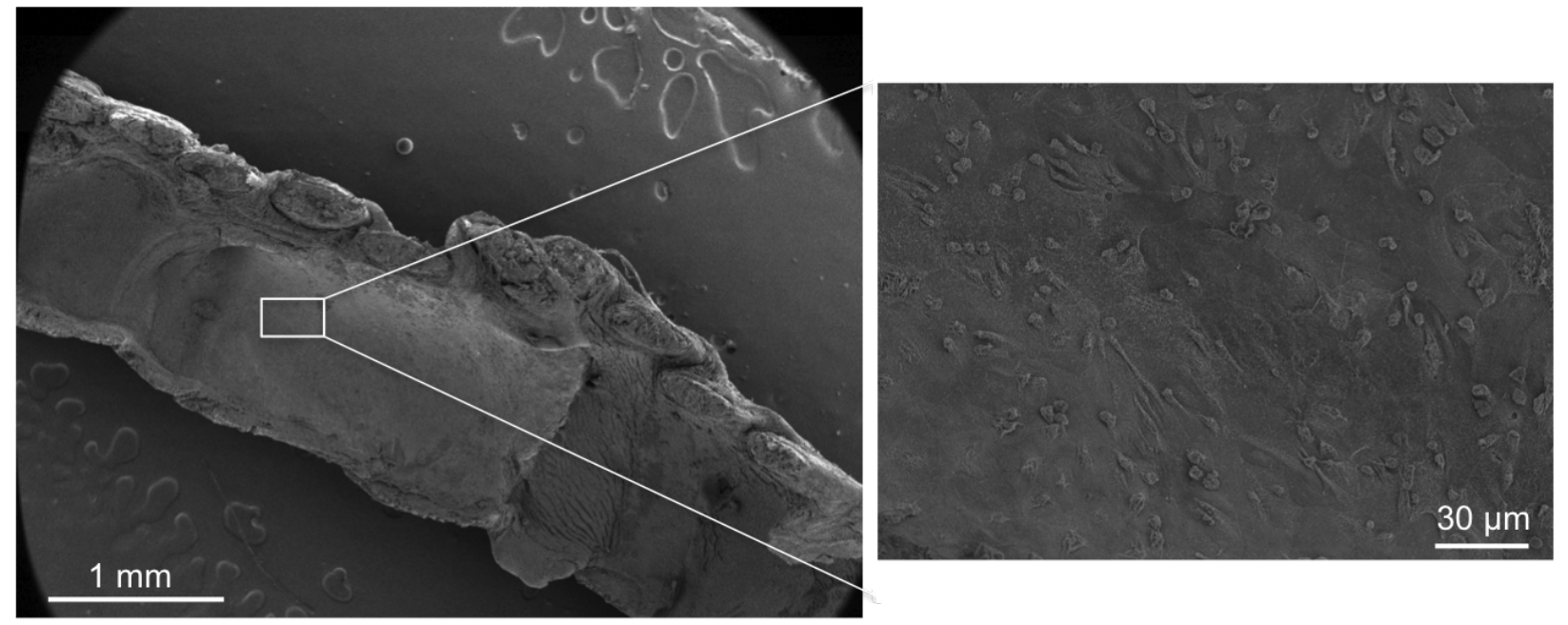

Figure 3.8: Representative scanning electron micrograph (25X) of 12 week CHAPS explant. Right frame shows expanded view (500X) of squamous epithelium 

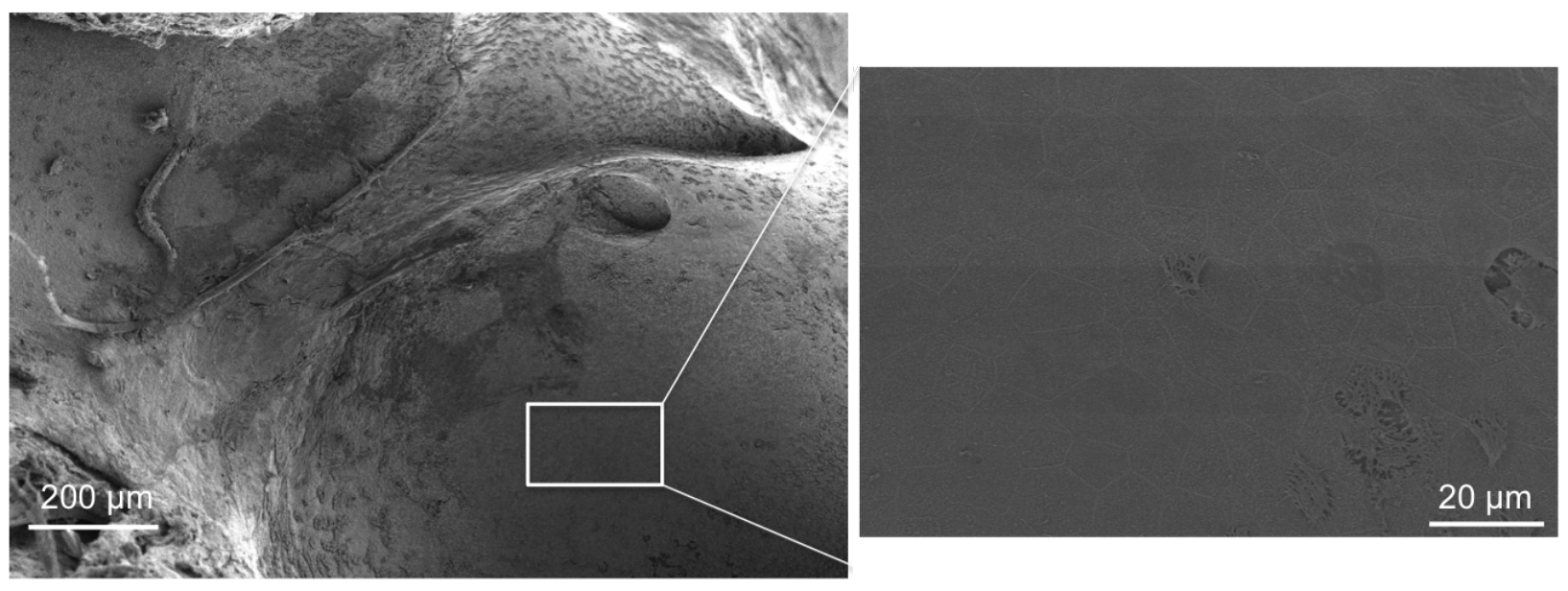

Figure 3.9: Representative scanning electron micrograph of 12 week SDS explant. Left: 100X magnification Right frame shows expanded view (1,000X) of squamous epithelium with several ciliated cells in the bottom right corner

\subsubsection{PAA treated Grafts Regenerate a Functional Immunological Barrier}

In addition to it's vital mechanical function, the trachea also acts as the first immunological barrier to environmental pathogens. Mucous traps inhaled particles and the ciliated epithelium beat in concert to remove the foreign particles (Figure 3.7). Therefore, to quantify the success of our implants, we must assess their function as an immunological barrier. Unfortunately, current techniques used to study airway cilia motility are limited. Clinically, brush biopsies and simple transverse airway sections are utilized, but these techniques are not viable in rodent specimens due to excessive damage upon removal. Alternatively, some groups employ various cell culture techniques to obtain sheets of differentiated ciliated epithelia from harvested airway cells. This technique requires significant time and the relevance to in vivo performance is questionable. Real time DIC imaging allows quick and simple determination of airway cilia function in a large number of different treatment groups. While being an in vitro technique the airway epithelium remains attached with its underlying and supporting 

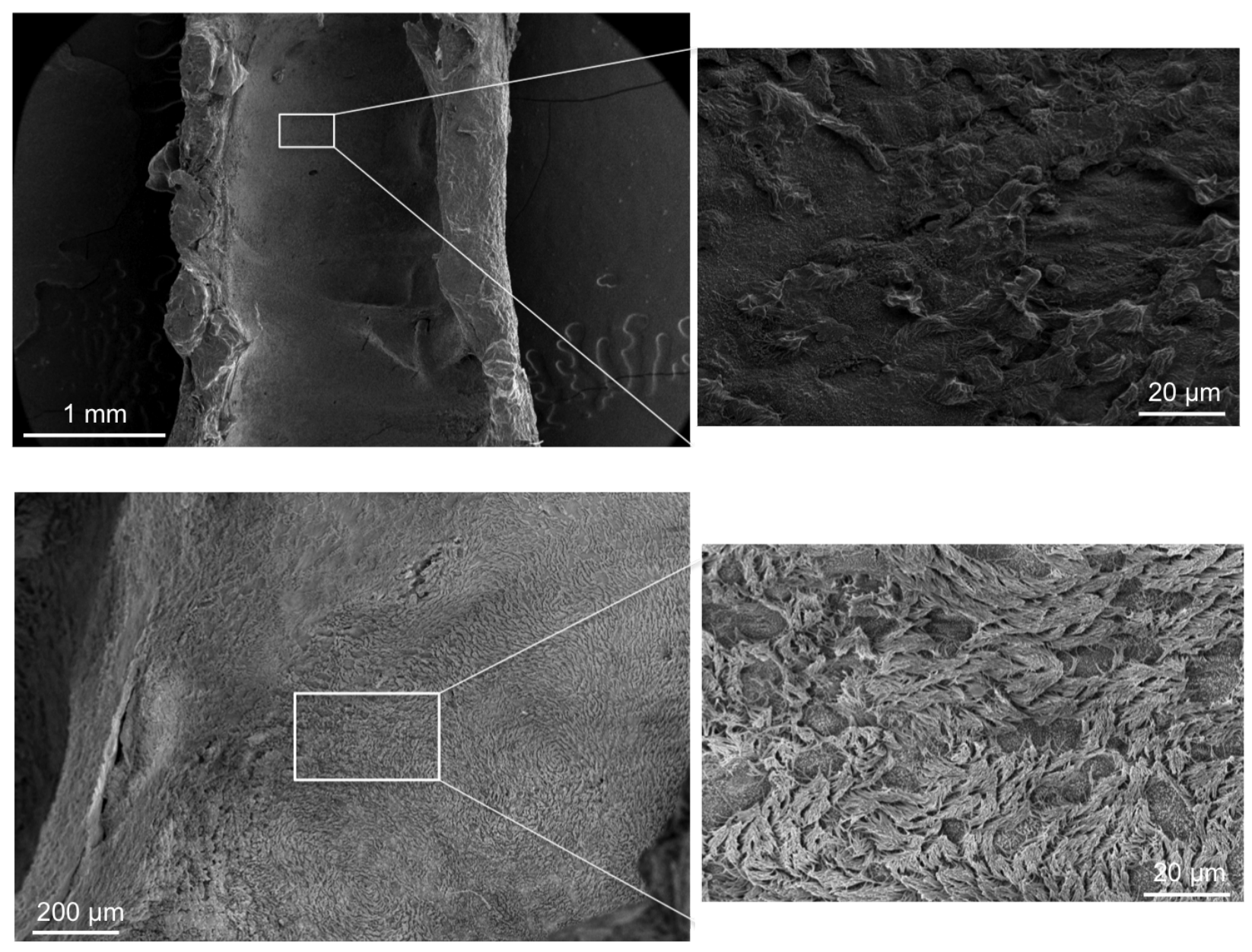

Figure 3.10: Representative scanning electron micrographs of 12 week Triton X-100 explants with expanded views. Top: Non-PAA treated control showing a patent lumen with good epithelial recolonization and differentiation. Bottom: PAA treated Triton X-100 graft showing excellent epithelial coverage with mature cilia. 

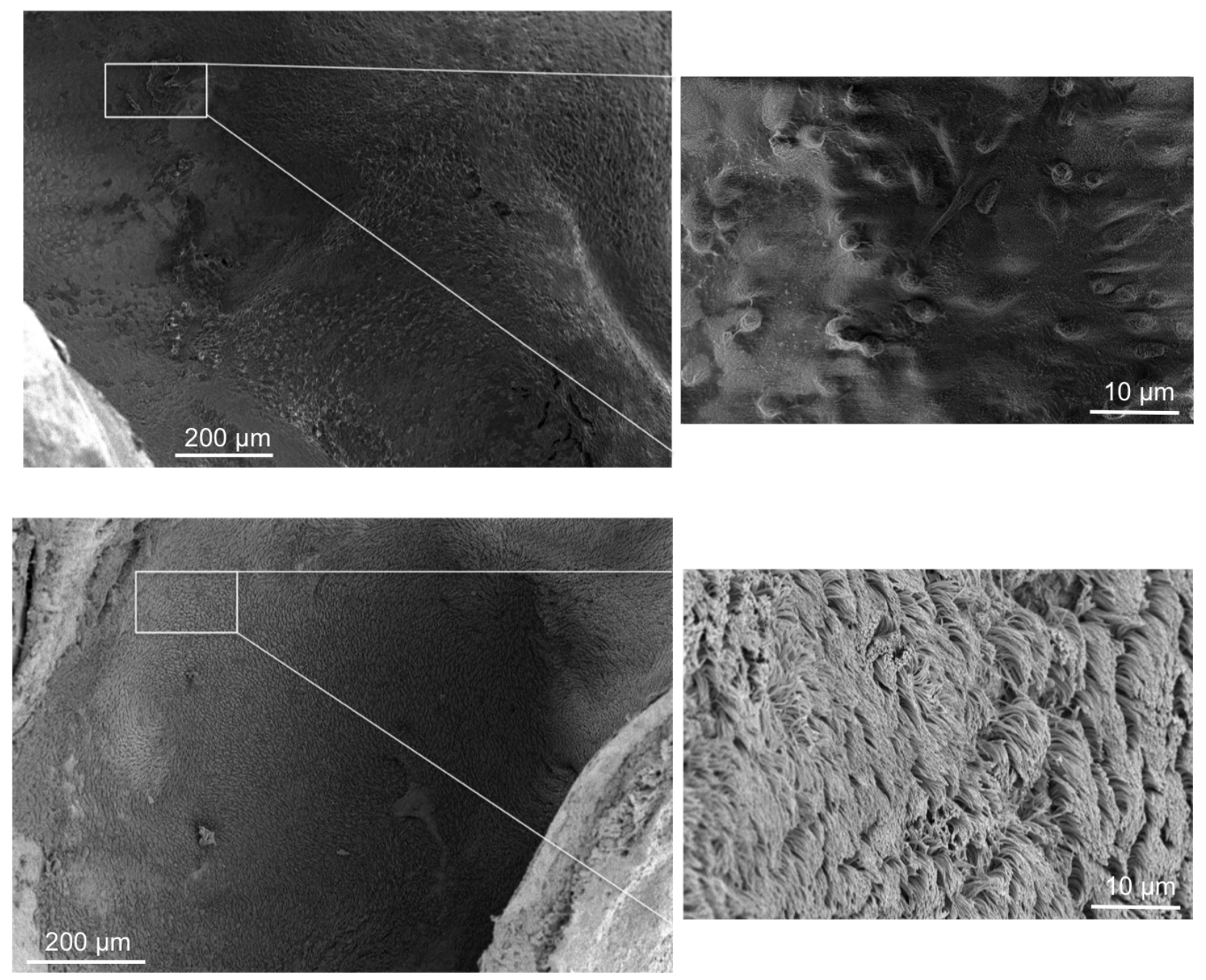

Figure 3.11: Representative scanning electron micrographs of 12 week sodium deoxycholate explants with expanded views. Top: Non-PAA treated control showing squamous epithelium with distributed patches of bare ECM (100X) . Bottom: PAA treated Triton X-100 graft showing excellent epithelial coverage with mature cilia. Right frame shows expanded view of squamous epithelium 


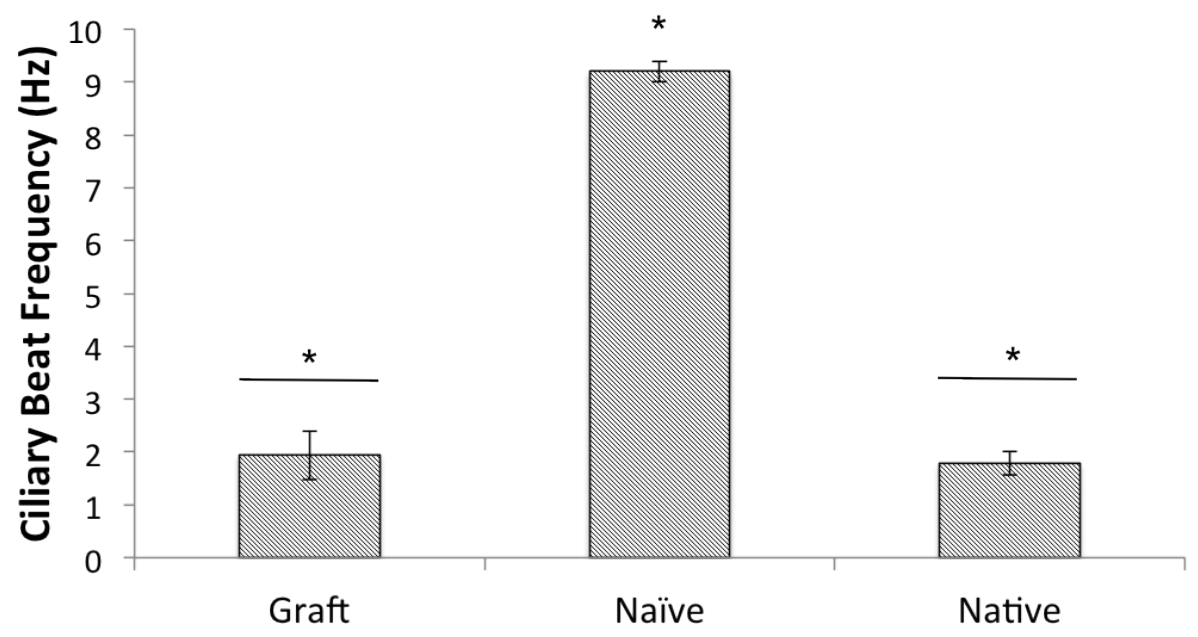

Figure 3.12: Ciliary beat frequencies computed from 12 week non PAA treated Triton X-100 explants $(\mathrm{n}=5)$. Explanted tracheal sections, both graft and native, have cilia function significantly lower than naive controls.

tissues which closely mimics its natural in vivo tissue environment.

Upon DIC imaging, it became clear that among the non PAA treated grafts, only Triton X-100 showed any significant cilia function. The sparse ciliation observed with SEM among SDS, CHAPS, and sodium deoxycholate groups was confirmed with DIC microscopy. The patchy regions of motile cilia in SDS and sodium deoxycholate grafts was insufficient for analysis. The non PAA treated Triton X-100 grafts showed beat frequency similar to the flanking native segments, however, these values were significantly depressed compared to naive controls (Figure 3.12). Remarkably, both Triton X-100 and sodium deoxycholate grafts treated with PAA showed ciliary beat frequency comparable to both flanking native sections and naive controls (Figure 3.13). Unfortunately, we were not able to preserve specimen orientation during imaging, therefore, we are unable to quantify global beat orientation. However, it is possible to qualitatively assess the beat coordination and uniformity of beat direction. It is clear within PAA treated groups there is significant coordination among neighboring cells, with PAA treated Triton X-100 grafts 


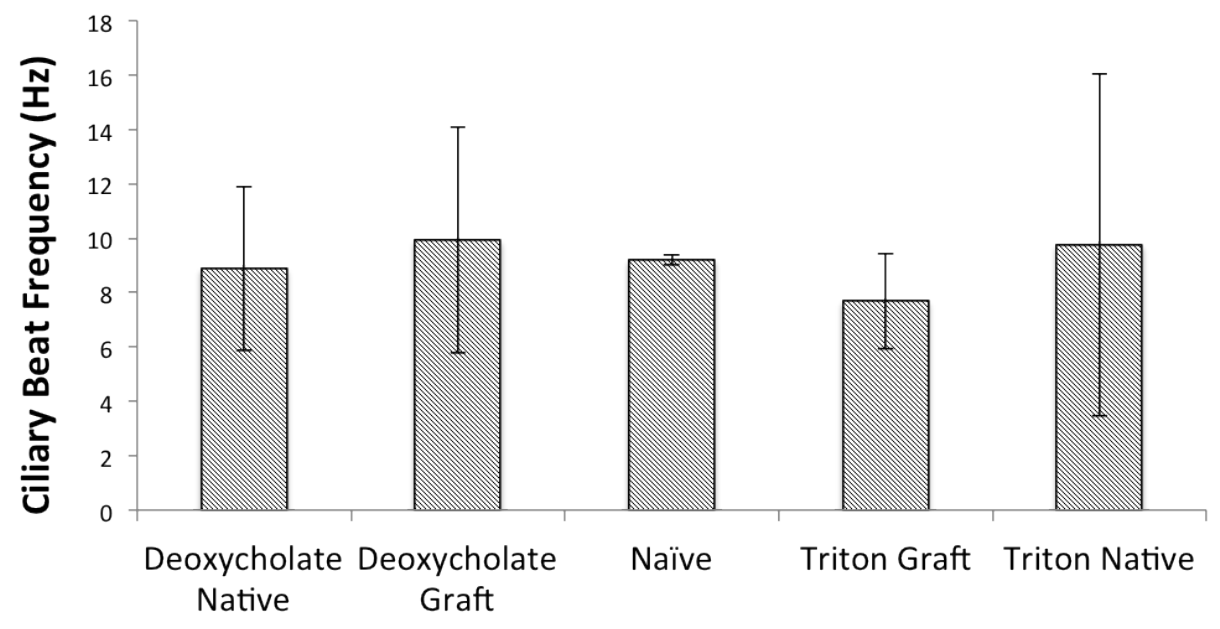

Figure 3.13: Ciliary beat frequencies computed from 12 week PAA treated Triton X-100 and sodium deoxycholate explants $(\mathrm{n}=5$ each). Naive cilia function is restored among all PAA treated grafts

demonstrating the longest range cilia coordination. Further work could be conducted to quantify this finding with particle image velocimetry (PIV) techniques. In this approach, tracer particles, are dispersed within the fluid and their motion is correlated to fluid flow characteristics. Since fluorescent particles may be employed within our existing setup, we could utilize lower magnification videos to gain longer range data on flow patterns and velocity rather than simply ciliary beat frequency. This approach has been previously employed in mutant murine models to assess features of cilia development and function, but would be a novel addition to the tissue engineered literature [211]. Regardless of our inability to quantify the fluid transport, this report is the first demonstration of cilia regeneration and restoration of native-like function within a tissue engineered transplant. 


\subsubsection{Limitations and Future Work}

In this study, we employed a relatively short graft, which in clinical settings would have simply been reanastomosed. While we suggest that the lack of initial cell seeding may improve outcome by temporarily slowing healing, the lack of an antigen barrier immediately at implant is a potentially serious concern. Given that there were no infections detected in over 30 animals, it seems that suitable prophylactic antibiotic administration can significantly hedge this risk. Unfortunately, the progression to a larger graft may require us to reevaluate this risk and develop more sophisticated approaches. Another major concern which has been partially addressed in previous section is our inability to specifically identify the mechanism of PAA improvement. Further studies are necessary to improve upon the in vivo analysis through more mechanistic studies of tracheal reepithelialization. For example, a recent study from our group has examined proliferation of various populations of tracheal basal cells over time [212]. The kinetics of epithelial healing was shown in murine tracheal reconstruction via immunofluorescent labeling of $\mathrm{K} 5+/ \mathrm{K} 14+$ basal cells. Histological data demonstrates a correlation between the depletion of the $\mathrm{K} 5+/ \mathrm{K} 14+$ cell population and the generation of a mature differentiated epithelium (Figure 3.13 and 3.14). This finding confirms other studies which have shown that $\mathrm{K} 5+/ \mathrm{K} 14+$ basal cells represent a precursor cell population with the capacity to develop into ciliated $(\mathrm{ACT}+)$ and secretory $(\mathrm{CCSP}+)$ cells. Potentially, this more detailed approach could shed light on the relationship between altered scaffold basement membranes seen in specific aim 1, differential basal cell proliferation and eventually epithelial differentiation in vivo.

\subsection{CONCLUSIONS}

It was found that there were dramatic differences in vivo among the various detergent de-

cellularized groups. Bearing in mind that the current clinical protocol employs sodium deoxycholate, these findings are extremely concerning. Of the tested specimens sodium deoxycholate performed among the worst. Our hypothesis that treatment with milder non-ionic 

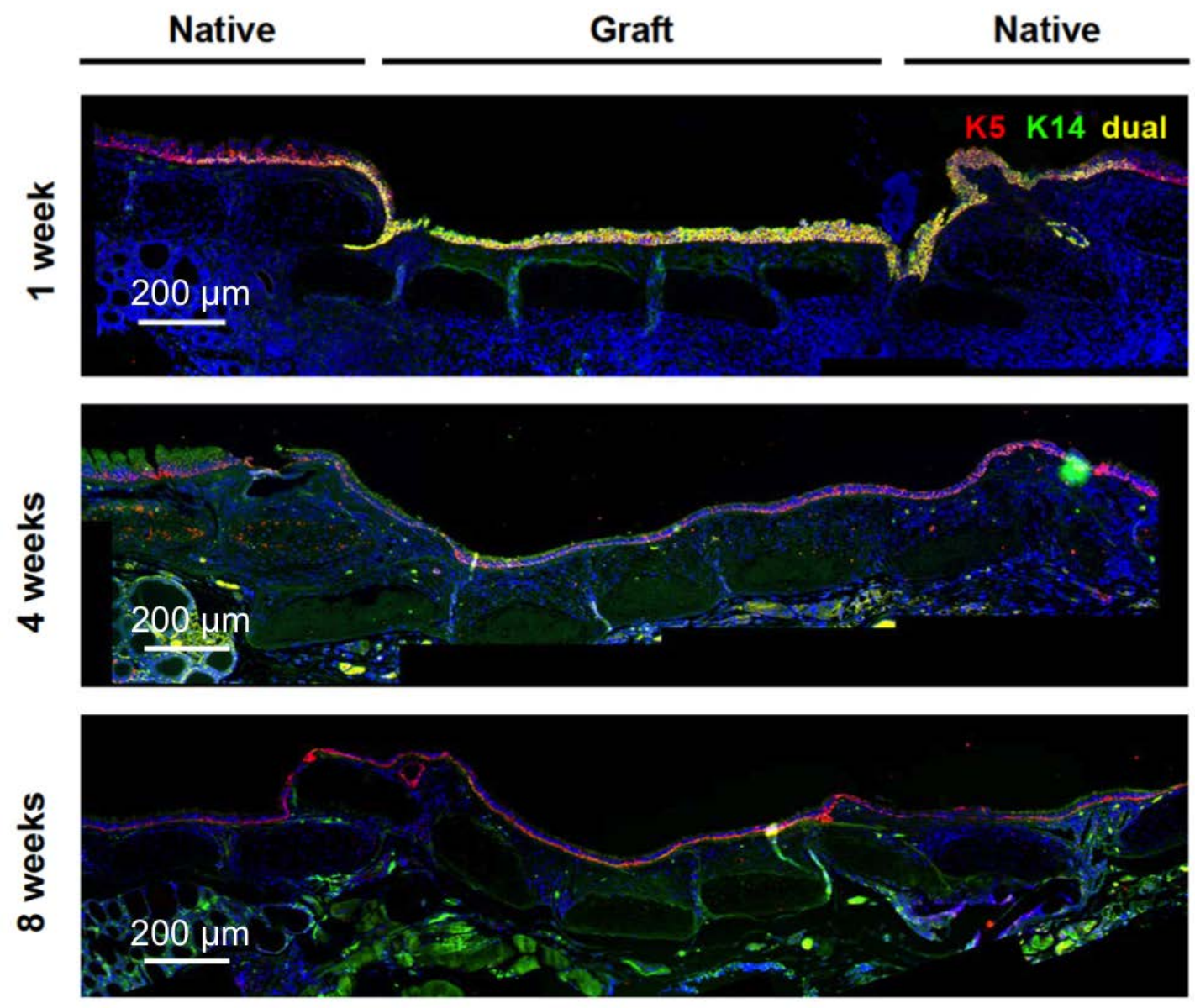

Figure 3.14: Histologic examination of basal cell protein expression within decellularized mouse tracheal grafts. Tracheas were explanted at 1, 4, and 8 weeks following surgery and were examined for expression of keratins 5 (K5, red) and 14 (K14, green), followed by DAPI staining (blue). Dual-positive cells are highlighted in yellow. Scale bar 200 microns. 

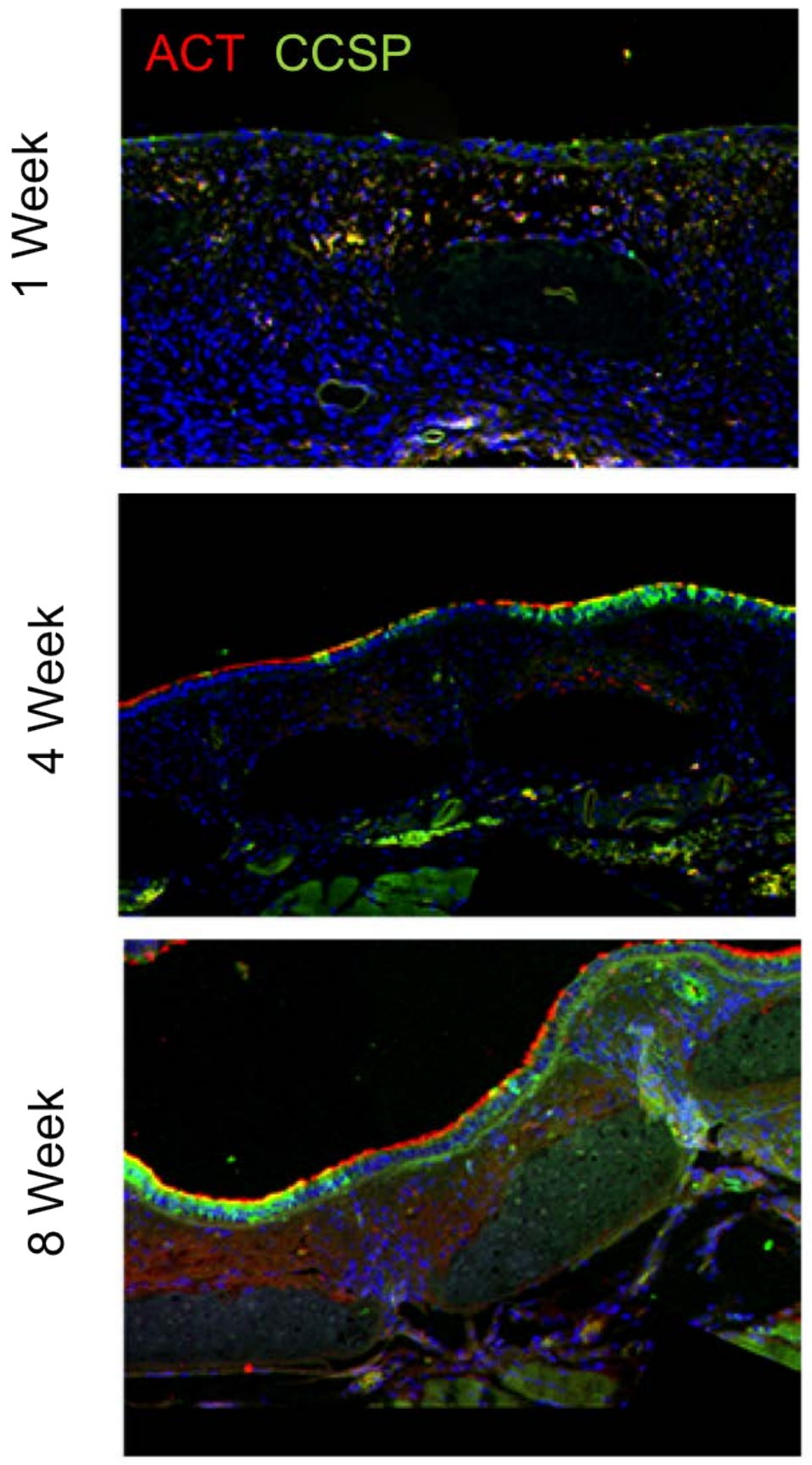

Figure 3.15: Explants of mice one, four, or eight weeks following surgery and immunofluorescent labeling for markers of mature epithelial cells was performed, followed by DAPI-staining (blue). Images are shown at a mid-graft location. Cells expressing acetylated tubulin (ACT, red) and Clara cell secretory protein (CCSP, green) are shown 
or zwitter-ionic detergents would improve host mediated remodeling was partially confirmed. Triton X-100 seemed to perform the best among the non PAA treated specimens, however, CHAPS performed most poorly, potentially due to it's weak amphiphilic character or it's

small micelle size. Our other hypothesis suggesting a degradation in scaffold performance with PAA post treatment was strongly refuted. It was found that PAA treatment significantly improved epithelial differentiation and restored a native-like immune barrier. In conclusion, it's clear that simple divergence from native structure is not a good metric for predicting scaffold performance. Second, simply picking an appropriate detergent is insufficient to ensure a positive implant outcome. While our current study is unable to establish a clear mechanism for this result, the empirical results are impressive. There are likely to be further challenges when translating to a large mamlian trachea, but our results suggest that employing Triton X-100 with a PAA post-treatment may dramatically improve the performance of the ECM implants through improvements in graft revascularization. Therefore, in specific aim 3 we will endeavor to translate this approach to a relevant large animal model.

\subsection{ACKNOWLEDGEMENTS}

We would like to thank Dr. Richard Francis for his assistance with CBF analysis and Dr. Nicholas Amoroso for his assistance with SEM preparation and analysis. 


\subsection{TRANSLATING TO A CLINCIALLY RELEVANT XENOGENEIC SCAFFOLD}

\subsection{INTRODUCTION}

Preliminary studies have demonstrated the use of decellularized human trachea clinically with excellent short-term results [148]. In this approach, cellular material was removed from a human donor trachea with detergent and enzymatic rinses. The remaining extracellular matrix (ECM), was then reseeded with the patient's own cells and implanted. Unfortunately, wider adoption of this approach has been hampered by significant donor tissue limitations, which has only been exacerbated during the past decade due to the increased incidence of vital organ failure. The increasing shortfall of adequate organs, largely driven by an aging population, has resulted in major organ shortages, not only significantly increasing in the number of patients on transplant waiting lists, but also wait time and mortality. In the United States, for example, a patient is added to the organ waiting list every 10 minutes. On average 79 people receive organ transplants daily, however, 18 people will die waiting for those same transplants [213]. Given this critical dearth of suitable human tissue for transplantation, it's not surprising that there is even less human donor tissue available for use in the wider adoption of the current clinical tissue engineering paradigm. In the infancy of the tissue engineering field, this limitation became immediately obvious and significant work was attempted to assess the feasibility of polymeric scaffolds and ECMs derived from xenogeneic sources. In the case of xenogeneic ECMS, thankfully, it was found that with proper processing conditions they were well tolerated during transplant and applied with enormous success to repair damaged tissues such as muscle[214], epidermis [215], bone [216], and tendon [217]. This success is evident in the extraordinary growth rate in the number of 
procedures. By 2002 more than 200,000 human patients had been implanted with xenogeneic ECM scaffolds [218]. This number quickly grew to exceed 1 million patients by 2007 [219].

Since the end-goal of this study is to engineer a decellularized porcine trachea suitable for human transplantation, we propose translating the optimum protocol identified in specific aim 2 (Triton X-100 with a PAA post treatment) to a clinically relevant porcine scaffold. Due to the obvious size differences among species it is not necessarily possible to directly translate an existing protocol. Often, translation requires harsher treatments or longer contact times, potentially negating the improvements produced as a result of optimized processing. Therefore, a previously developed novel cyclical pressure device will be implemented to aid in the most direct translation to porcine tracheal tissue.

\subsubsection{Cyclical Pressure Decellularization}

Since its inception as a field, there has been significant work to characterize the structure and biology of ECM scaffolds and their effect in vivo; yet, the chemicals and techniques employed during decellularization have remained relatively unchanged. There have been several novel approaches employing using ultrahigh pressure [220] or even supercritical carbon dioxide [221]. However, these have shown minimal improvements for the substantial increase in complexity and cost. Other than hypo- and hypertonic solutions, traditional treatments do not dramatically improve perfusion into and out of the tissue. Therefore, cyclical hydrostatic pressure was tested to improve infiltration of decellularization chemicals and removal of cellular debris. In order to test this hypothesis, a device was assembled from commercially available parts including a vacuum pump, cryotrap, pressure chamber and solenoid valve (Figure 4.1). Custom LabView software was written to control the rotary vane vacuum pump and 3-way solenoid valve. Cycle rate, number, and duty cycle were controlled independently. Pressurization and depressurization rates also were controlled through the proportional solenoid valve. Through extensive empirical testing, it was found that when tissues were submerged in decellularization solutions and exposed to negative pressures with high pressure ramp rates the efficacy of standard decellularization chemicals was dramatically improved. To demonstrate the efficacy and logic of this approach and we will present 
three studies. Two explore the retention of native tissue mechanics during extended decellularization of dermis and vocal fold compared to traditional agitation controls. The third, demonstrates the ability of cyclical pressure decellularization to fully decellularize aorta and successfully perform in long-term large animal implants.

\subsubsection{Pressured Assisted Decellularization Improves Retention of Native Me- chanical Properties}

Porcine Dermis. The thickness, density, and complexity of the dermis has historically required the use of a variety of mechanical and chemical methods during tissue harvest and decellularization. Hot water sanitation and liming are common practices prior to decellularization. Hot water treatment sanitizes the skin and aids in hair removal, while liming serves the dual purpose of disinfection and hair removal. Further decellularization steps have employed harsh enzymatic treatments with trypsin and long contact times with strong detergents such as SDS [222]. We have attempted to demonstrate that none of these steps are necessary with suitable perfusion resulting from cyclical pressure decellularization. In our study porcine dermis was obtained in PBS at $4^{\circ} \mathrm{C}$ from Tissue Source. The tissue was then frozen at $-80^{\circ} \mathrm{C}$ overnight. Dermis was thawed in DI water and subjected to decellularization with 60 second alternating vacuum cycles submerged in DI water, $3 \%$ Triton X-100, $3 \mathrm{M} \mathrm{NaCl}$, then 2,000KU DNAse each for 30 minutes. This process was repeated twice, then the specimens were rinsed overnight in $\mathrm{PBS}$ at $4^{\circ} \mathrm{C}$. This whole protocol was conducted 7 more times. Traditional H\&E sections of the specimens were obtained to evaluate structure and removal of intact nuclei and cellular debris with the cyclical pressure decellularization approach. As we can see from Figure 4.2, a 7 day decellularization protocol employing these steps appears to be capable of complete nuclear removal with almost no structural disruption. To confirm the structural findings observed in histological sections, we sought to obtain mechanical properties in response to a ball-burst test of native, agitation control, and vacuum decellularized dermis. Specimens were cut into square samples $25 \mathrm{x} 25 \mathrm{~mm}$ and examined using an American Society of Testing and Materials (ASTM) standard ball-burst apparatus with a ball diameter of $9.52 \mathrm{~mm}$ and a sample surface area of $216 \mathrm{~mm}$. The tissues 

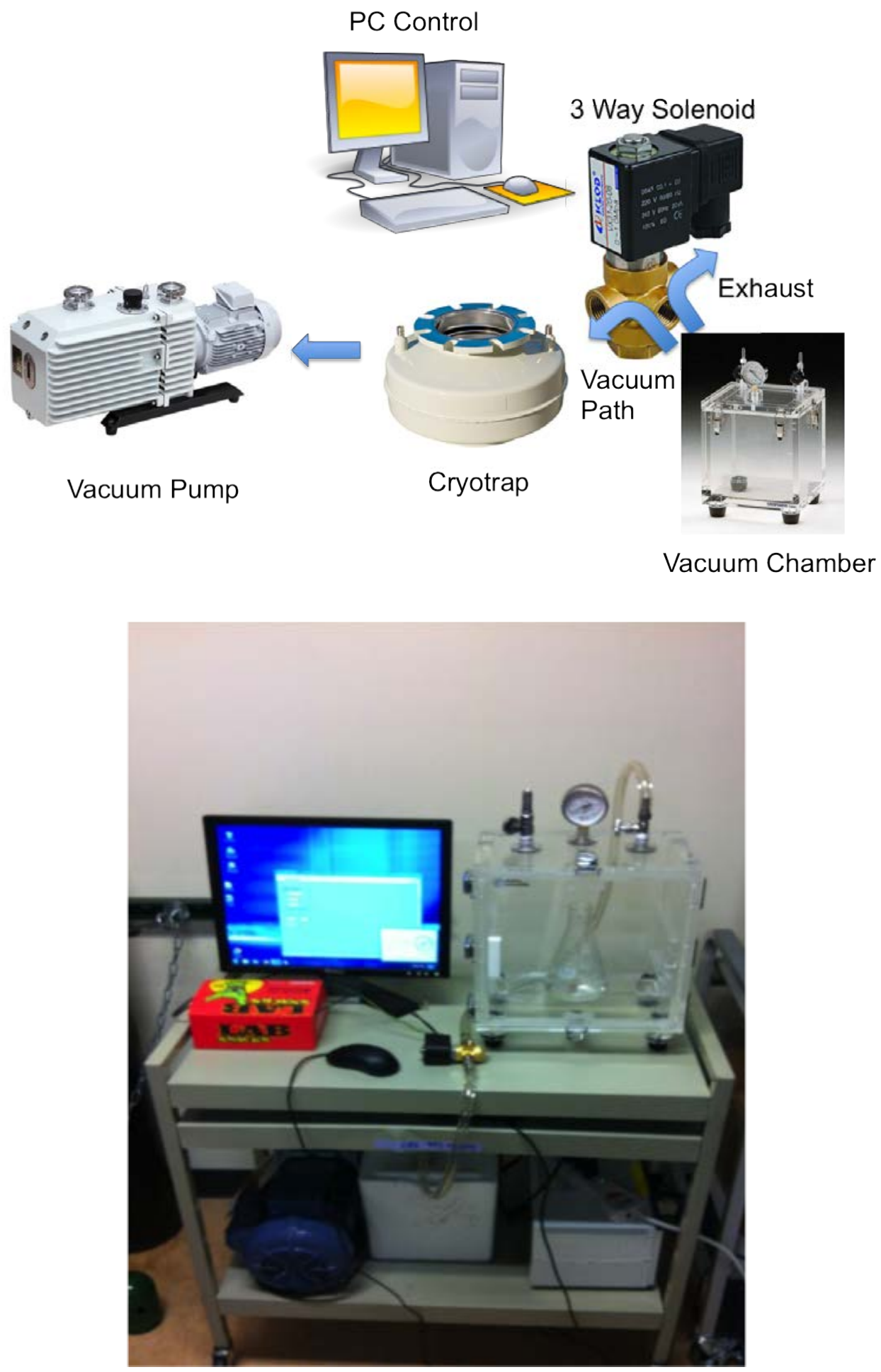

Figure 4.1: Cyclical pressure device comprised of a PC controlled vacuum pump, solenoid valves, pressure sensor, and vacuum chamber. Pressure is cyclically pulsed with tissue submerged in a detergent to effect decellularization 


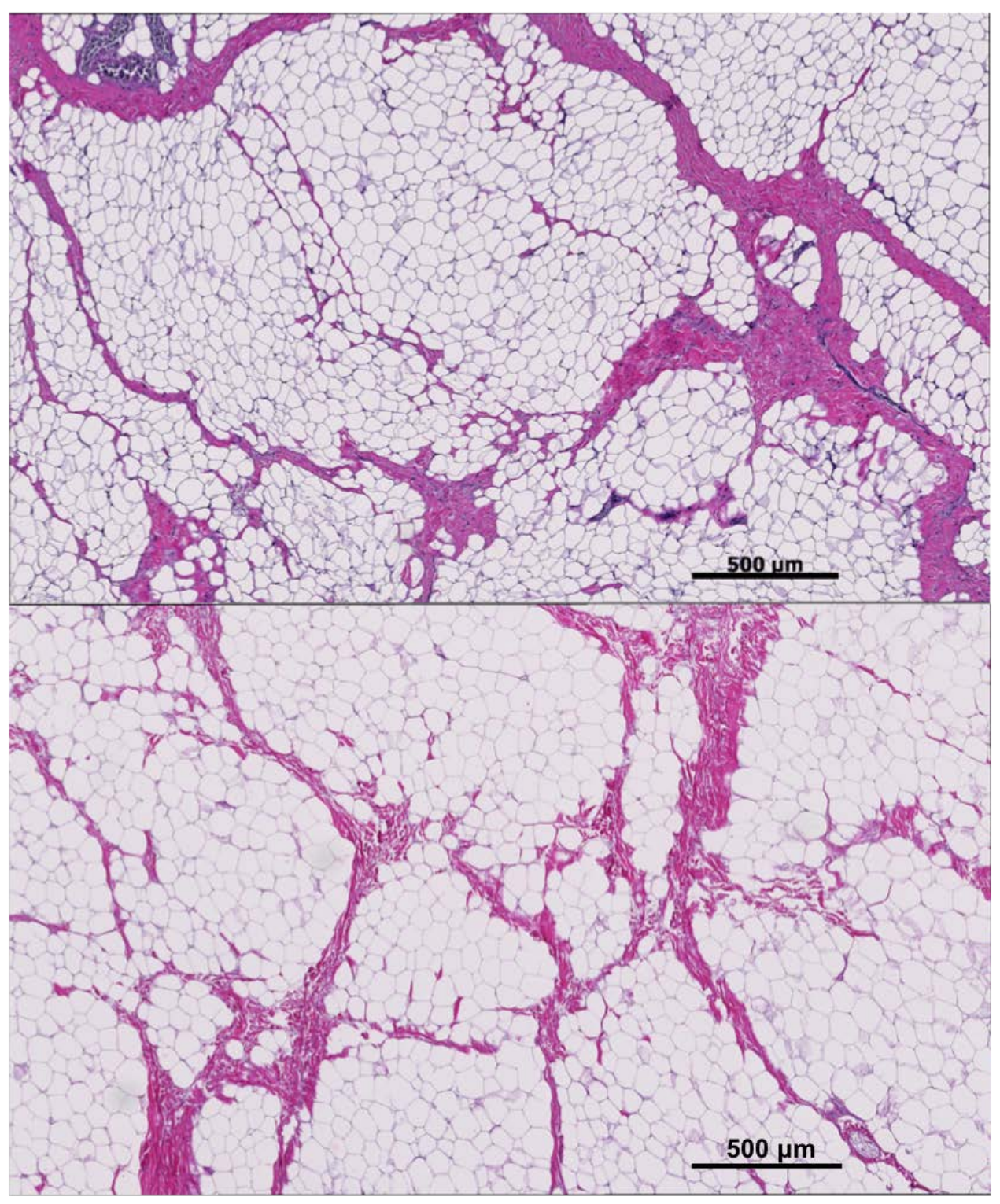

Figure 4.2: H\&E sections of: Native porcine dermis (Top) and Cyclical Pressure Decellularization (Bottom) 
were secured between two flat clamps with interlocking triangular grooves and mounted onto a custom stand, which attaches to the base of an Instron 4502 (Instron, Norwood, MA). The ball was connected in series with a load cell (Honeywell, $5 \mathrm{kN}$ ) and a movable cross-head. Prior to testing, a small pre-load of $1 \mathrm{~N}$ was applied to ensure contact between the dermis and the ball while minimizing tissue deformation. Each sample was then loaded to failure at a rate of $10 \mathrm{~mm} / \mathrm{min}$. The resulting load-elongation curves were analyzed to determine the structural properties of each tissue. Failure load (N) and maximum extension (mm) corresponding to the ball breaking through the specimen were recorded. To calculate stiffness $(\mathrm{N} / \mathrm{mm})$, the maximum slope over a running window of $20 \%$ of the failure elongation was used. As evident in Figure 4.3, there is a significant retention of native mechanics in the vacuum decellularized scaffolds compared to agitated controls, confirming our original hypothesis.

Porcine Vocal Fold. Unlike dermis, the vocal fold's function is dependent on a series of very fine and highly specific mechanical properties. Past studies raise concern that the use of harmful chemical and mechanical agitation disrupts the internal matrix structure, causing the scaffold to become significantly more elastic than the properties of native tissue. Thus, the current generation of decellularized vocal fold scaffolds do not possess the correct viscous mechanical properties and over-exaggerate vibratory functions [223]. As with the dermis, our goal with vocal fold was to assess the relative retention of appropriate mechanics during decellularization. To this end, we employed linear shear analysis, which is a physiologically relevant testing modality that mimics the vibratory function of the vocal folds.

Larynxes were harvested and received from TissueSource. Tissues were rinsed with Type I water and frozen at $-80^{\circ} \mathrm{C}$ until use. Vocal folds were isolated from the larynx in approximately $6 \mathrm{~mm}$ by $6 \mathrm{~mm}$ sections using a scalpel and forceps. Each specimen was subjected to three 10 minute washes of Type I water, 3\% Triton X-100, and 3M NaCl. During each wash the vacuum chamber containing the specimens was evacuated for 30 seconds at 1 minute intervals. Between each 10 minute cycle, specimens were rinsed with Type I water. Between vacuum treatments, tissues were stored at $4^{\circ} \mathrm{C}$ on an orbital shaker at $200 \mathrm{rpm}$ in solutions of Type I water, $3 \%$ Triton $\mathrm{x}-100$, and $3 \mathrm{M} \mathrm{NaCl}$ for each respective night. Lasting for 4 days, the protocol exposed tissues to 12 cycles of all three solutions. Upon comple- 


\section{Maximum Stiffness}

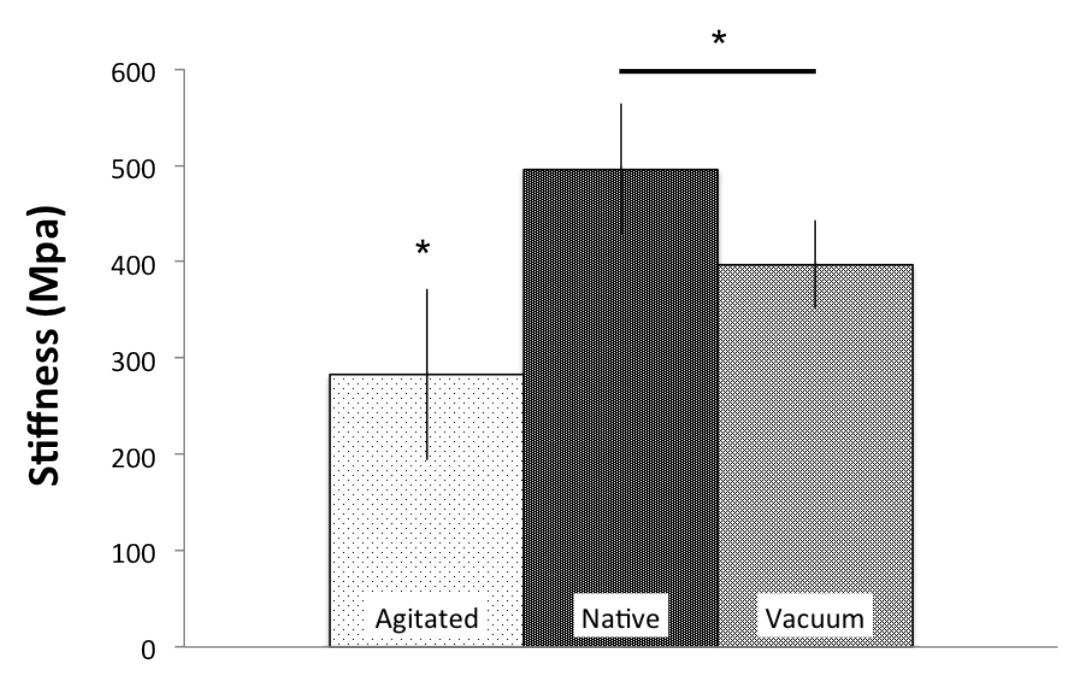

Failure Stress

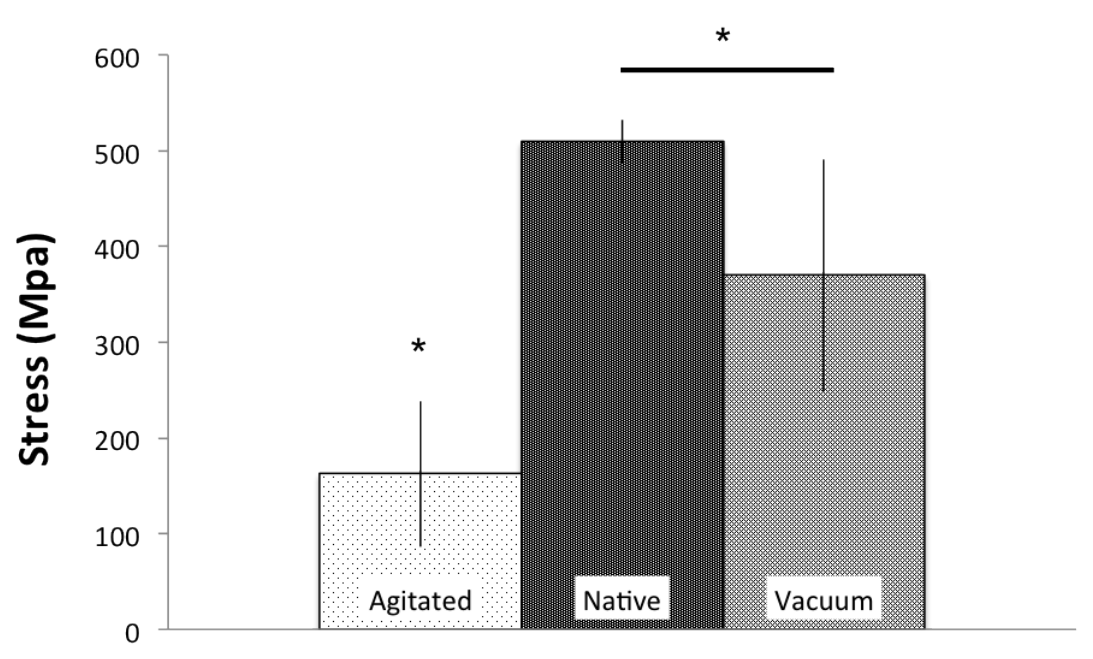

Figure 4.3: Plots comparing maximum stiffness and failure stress for cyclical pressure decellularized, agitation controls, and native vocal folds. Please note the significant difference between agitation controls and both cyclical pressure decellularized and native tissue for both measured parameters. 
tion of vacuum decellularization, the remaining decellularized connective tissue matrix was characterized as VF-ECM. 0.1\% (v/v) peracetic acid was used to disinfect the tissues. The disinfected VF-ECM was washed twice for 15 minutes with PBS ( $\mathrm{pH} \overline{7} .4$ ) and twice for 15 minutes with Type I water. The VF-ECM was then packaged in physiologic saline and terminally sterilized through gamma irradiation. Assessment of the viscoelastic properties held by the cyclical pressure decellularized scaffold, the agitated control, and native tissue was completed via linear shear analysis using an EnduraTEC Electro-Force Mechanical Testing System (Model ELF 3200, Bose, Minnetonka, MN). Capable of strain oscillations of up to $200 \mathrm{~Hz}$, a frequency sweep was performed from 0.1 to $175 \mathrm{~Hz}$ in $5 \mathrm{~Hz}$ increments. Prior to applying any load, compression was set to $40 \%$ to ensure an adequate and representative tissue response. Properties of native tissue were collected to establish a baseline for comparison. Scaffolds were attached to the lower, stationary plate by mounting them on sand paper. As the upper plate oscillated, values of mechanical properties were assessed and collected within an Excel file. Upon completion of the data acquisition phase, the Excel files were sent to a Mathematica code, which characterized the viscoelastic properties of the specimen. Mann-Whitney statistics were conducted to assess significance between the mechanical parameters of native and decellularized tissues, significance was gained at $\mathrm{p}<0.05$. It was shown that traditional agitation based decellularization approaches were incapable of producing VF-ECM with appropriate mechanical properties, whereas, there were no significant differences between cyclical pressure decellularization and native specimens (Figure 4.4).

\subsubsection{Demonstrated Success of Cyclical Pressure Technique in Three Month Large Animal Implants}

Porcine Aorta. Given the benefits observed in other tissues known to be notoriously difficult to decellularize, it was felt that the cyclical pressure device could be beneficial if applied sucessfully to porcine aorta. In this study, we sought to demonstrate decellularization without the trypsin or other harsh detergents employed in other studies, which have

been shown to have a strong effect on the resulting mechanical properties [224, 225]. To 


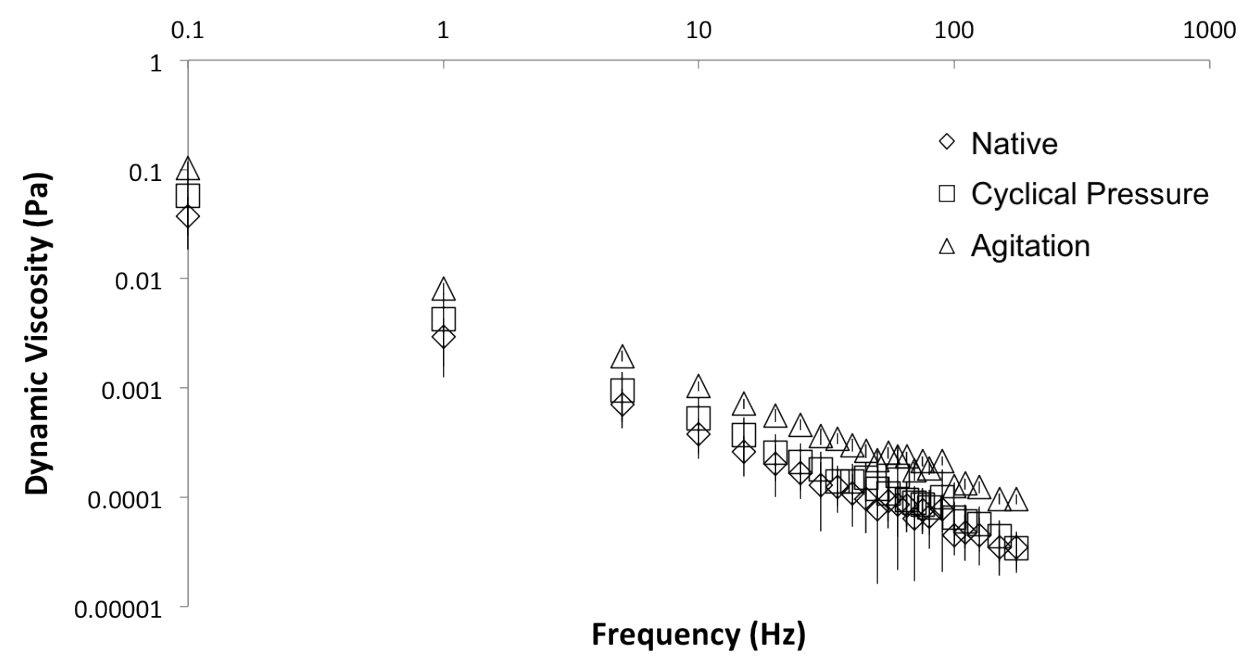

Figure 4.4: Viscoelastic properties of cyclical pressure decellularized, agitated controls, and native vocal folds. Agitated controls were found to be significantly different than both cyclical pressure decellularized specimens and the native control. No significant difference was observed between native and cyclical pressure decellularized specimens. 


\section{Native}

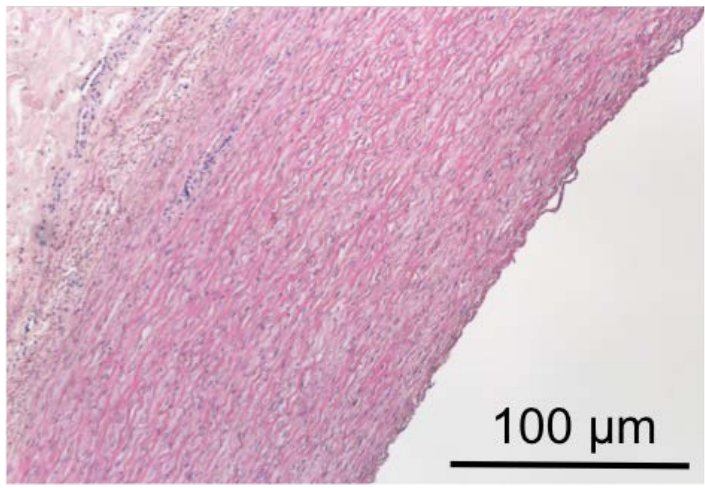

Agitated Control

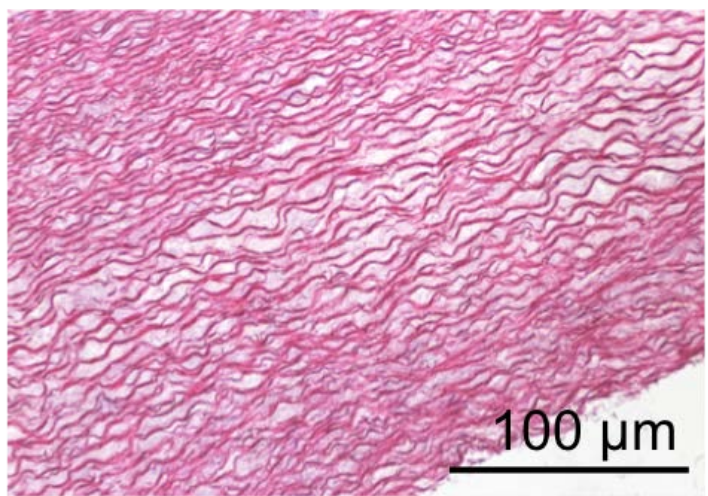

\section{Cyclical Vacuum Decellularization}

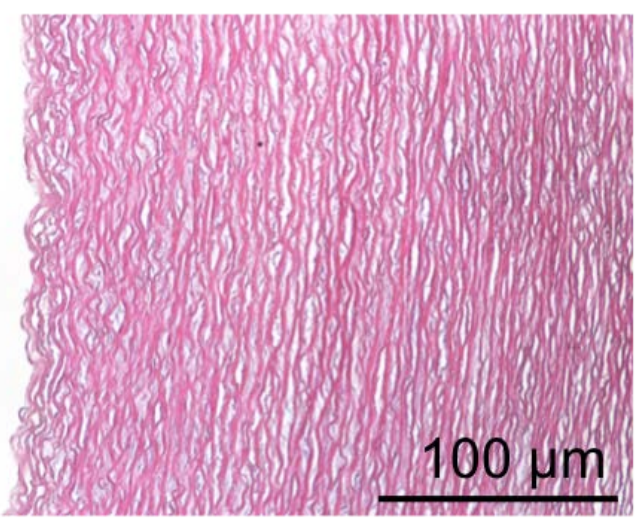

Figure 4.5: Comparison of hematoxylin and eosin staining for native, agitation control, and cyclical pressure decellularized porcine aorta demonstrating improved retention of tissue micro-structure in pressure assisted techniques compared to standard agitation decellularization. 
accomplish this, porcine aorta was obtained in PBS at $4 \mathrm{C}$ from Tissue Source. The tissue was then frozen at $-80^{\circ} \mathrm{C}$ overnight. The aorta was thawed in DI water and subjected to decellularization with 60 second alternating vacuum cycles submerged in DI water, $3 \%$ Triton $\mathrm{X}-100,3 \mathrm{M} \mathrm{NaCl}$, then 2,000KU DNAse each for 30 minutes. This process was repeated twice, then the specimens were rinsed overnight in PBS at $4^{\circ} \mathrm{C}$. This whole protocol was conducted 12 more times, to ensure complete decellularization as determined by DNA gel electrophoresis and histology (Figure 4.5). The aortic tissue was then packaged in physiologic saline and terminally sterilized through gamma irradiation. Patch aortaplasty was conducted on $15 \mathrm{~kg}$ piglets under anesthesia and aseptic conditions - shaved skin, sterile surgical fields, preparation with Betadine. A left thoracotomy was performed in the fourth intercostal space. Vascular clamps were placed across the descending aorta separated by 4 $\mathrm{cm}$. The aortic clamp was maintained for approximately 20 minutes. A 4cm long, 50\% circumferential segment of the descending aorta was resected and substituted with an arterial graft to repair the surgical defect with polypropylene 5/0 suture. The clamps were released with appropriate deairing of the anastamosis and hemostasis assured (Figure 4.6). The ribs were reapproximated with heavy vicryl suture, the lung fully expanded, and the peritoneal layer and skin closed in two layers. A chest tube was placed with a purse string suture, if necessary, and removed within 48 hours depending on drainage. After 12 weeks, animals were euthanized and the aorta was excised with several $\mathrm{cm}$ of flanking native tissue. At explant, the patches had become remarkably well integrated with no thrombosis or evidence of an ongoing inflammatory response (Figure 4.7). The patch had also appeared to have completely reendothelialized.

\subsection{MATERIALS \& METHODS}

\subsubsection{Tissue Collection}

The trachea from Whiteshire Hamroc pigs were harvested immediately following euthanasia (Tissue Source). The animals were of similar genetic heritage, and were raised and kept 


\section{Preimplant}

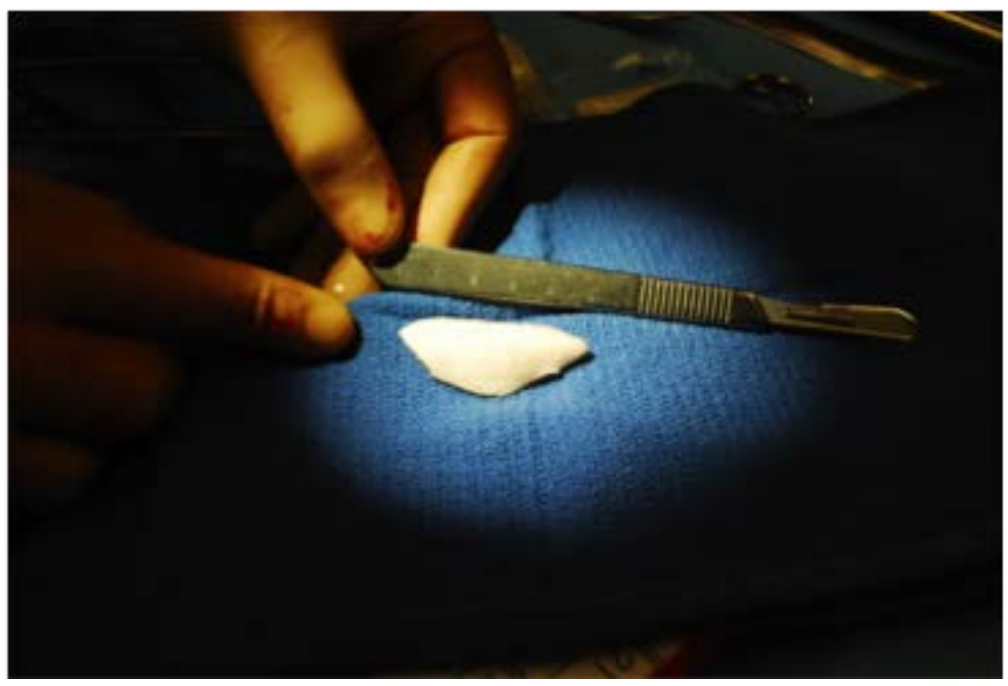

\section{Prior to Closure}

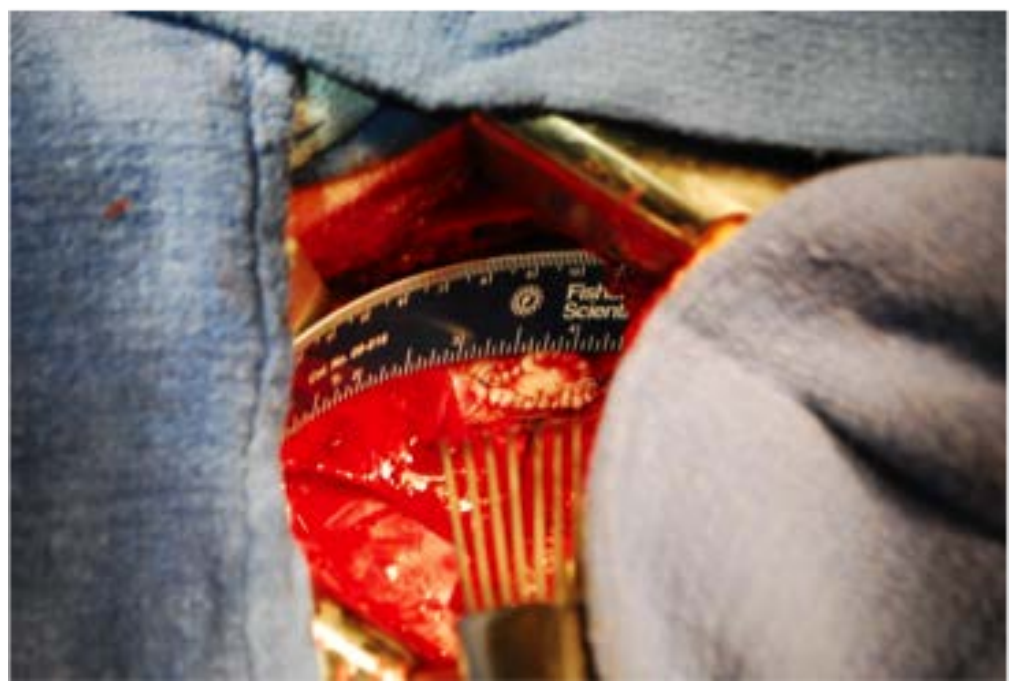

Figure 4.6: Surgical images demonstrating the graft prior to implant (top) and completed surgery immediately before closure (bottom) 

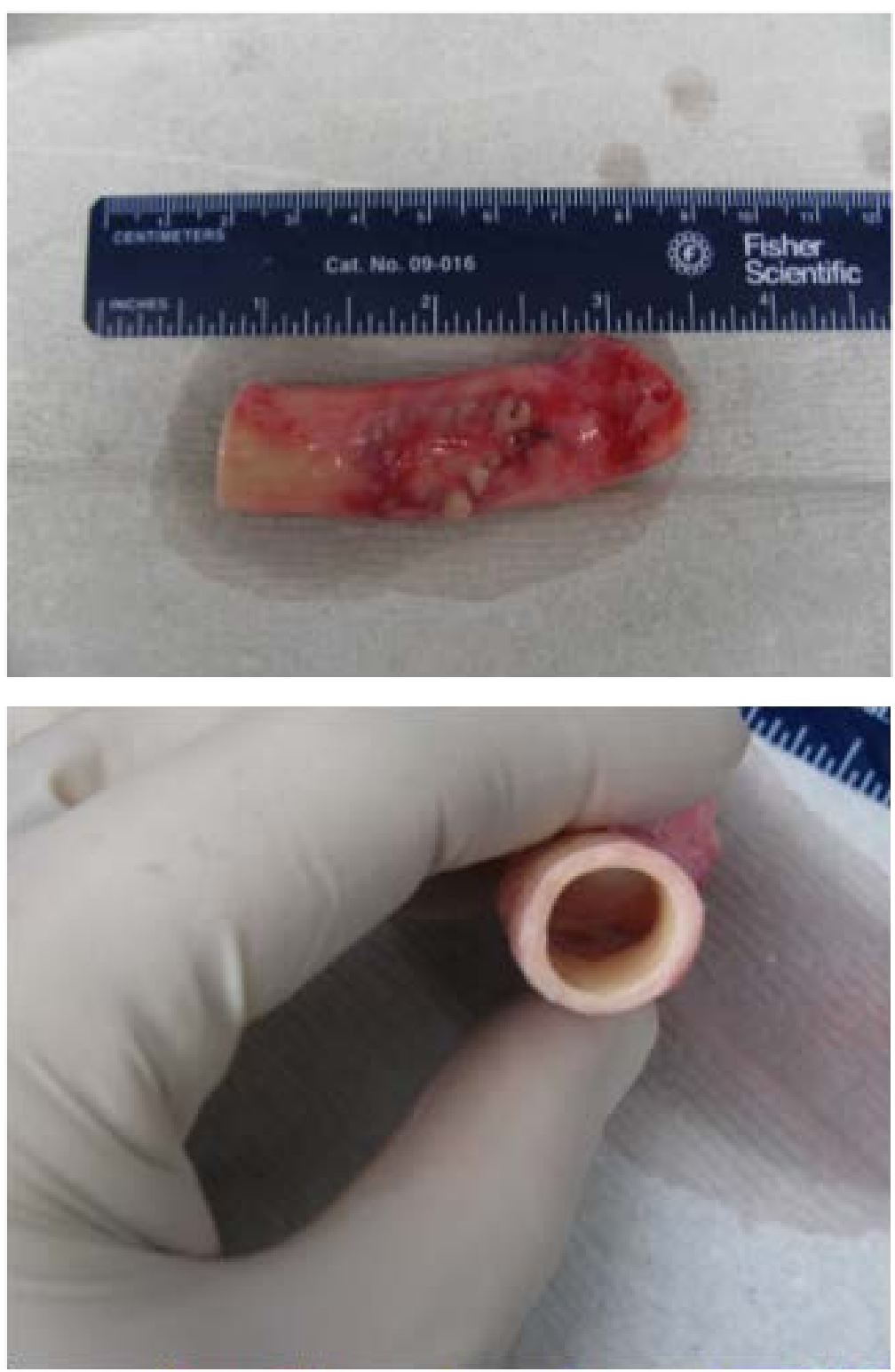

Figure 4.7: 12 week explanted cyclical pressure decellularized specimens 
in identical husbandry conditions including diet and vaccination history. All tissues were harvested on the same day and stored on ice during overnight shipping.

\subsubsection{Porcine Trachea Vacuum Decellularization}

Specimens were thoroughly rinsed with deionized (DI) water and cleaned of extraneous tissue under a Zeiss DV4 dissecting scope. Samples were frozen at $-80^{\circ} \mathrm{C}$ until use. Porcine trachea were decellularized in 14 days with overnight rinses. Each day, tracheas were submerged in four separate solutions (DI water, $3 \%$ triton X-100 3M, and 2,000 KU DNAse I in $1 \mathrm{M}$ $\mathrm{NaCl}$ ) each for 30 minutes. During submersion in each solution, the pressure within the surrounding chamber was cycled once a minute (evacuate chamber from $0.1 \mathrm{MPa}$ to $0.006 \mathrm{MPa}$ in 30 seconds, hold 25 seconds, pressurize $0.006 \mathrm{MPa}$ to $0.1 \mathrm{MPa}$ in 5 seconds, Figure 4.8). Overnight, specimens were stored in 1\% anti/anti in PBS. After 14 days of decellularization,

specimens were exposed to a single $0.1 \%$ PAA and $4 \%$ ethanol treatment for 90 minutes followed by three 30 minutes rinses in PBS. Finally, all trachea were individually packaged in physiologic saline and gamma irradiated to ensure terminal sterilization.

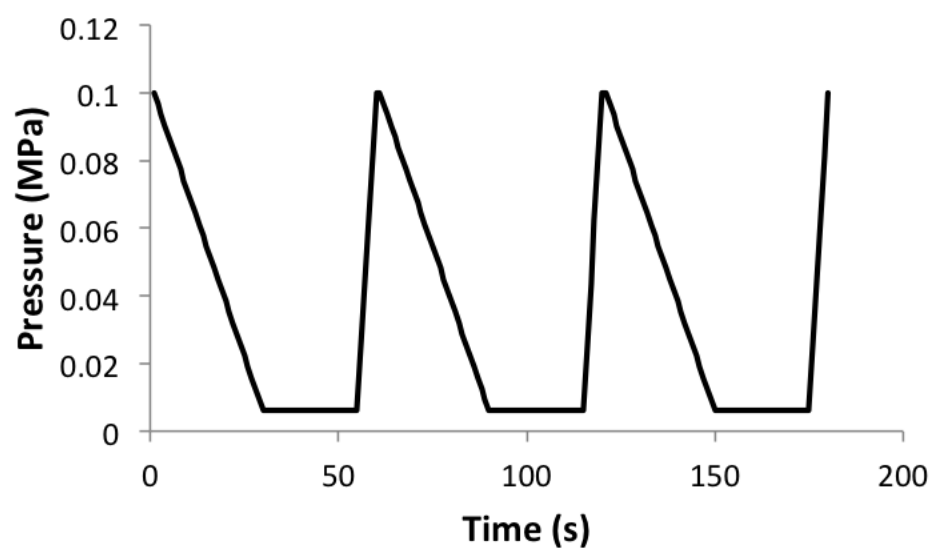

Figure 4.8: Chart demonstrating programmed pressure profile for three decell cycles taking approximately one minute each 


\subsubsection{Suture Retention Testing}

Tracheas were transversely dissected and reanastomosed using 2-0 chromic gut in 5 equally spaced, interrupted sutures. The proximal and distal ends of the trachea were clamped between between two flat clamps with interlocking triangular grooves (Figure 4.2). A uniaxial Instron 4502 (Instron, Norwood, MA) with 5kN load cell (Honeywell) and a moveable crosshead was employed for testing. Prior to testing, a small preload of $1 \mathrm{~N}$ was applied. Each sample was then loaded to failure at a rate of $50 \mathrm{~mm} / \mathrm{min}$. The resulting load-elongation curves were analyzed to determine the suture retention properties of the decellularized trachea compared to native controls. Average maximum failure load (N) was computed.

\subsubsection{Pressure Diameter Testing}

The effects of decellularization on the mechanical behavior of the decellularized graft was determined by performing pressure diameter testing employing the device described in Specific Aim 1. Three porcine tracheas were tested both before and after decellularization. Trachea were installed and retained on the notched adapter with zip ties. Upon mounting, a small tension was placed on the tissue along the length of the graft to simulate physiologic tension. Tracheas undwent 10 preconditioning cycles, from $-10 \mathrm{mmHg}$ to $60 \mathrm{mmHg}$ to simulate physiological respiration. Trachea were then tested quasistatically at -200 mmHg and 200 $\mathrm{mmHg}$ to confirm structural integrity at sub and supra-physiologic pressures [226].

\subsubsection{In vitro Cell Culture}

Human brochial epithelial cells were obtained from excess pathological tissue remaining after lung transplantation under a protocol approved by the University of Pittsburgh Investigational Review Board. Tissue was obtained following lung transplantation for a variety of pathological conditions including bronchiectasis, emphysema, primary pulmonary hypertension, pulmonary fibrosis, and alpha1-antitrypsin deficiency. Tissues was acquired by care-

fully removing 2-3 cm pieces of airway from lungs with sterile scissors and forceps. Excess parenchyma was removed and the airway opened lengthwise. The airway lumen was cleaned 
by wiping on the sterile field. The airway was then placed in sterile, cold EMEM/HEPES + sodium bicarbonate $\mathrm{pH}$ 7.41,8 (referred to as EMEM/HEPES) on rocker at 4C overnight. Media was then aspirated, excess tissue was removed from outside of airway, the tissue was then rinsed aggressively in EMEM/HEPES and blotted on a sterile towel. Tissue is then placed in 1mg/mL Protease media containing type XIV: Bacterial, From Streptomyces griseusin in EMEM/HEPES media for 36-48h at 4C. After culture, tissue in gently scraped with a scalpel blade. After scraping all tissue pieces, the petri dish is washed with fresh, cold EMEM/HEPES media to collect cells. Cells are spun down cells for 5 minutes at 900 RPM and resuspended in Accutase for 10 minutes on rocker at 4C. Accutase is supplemented with $1 \%$ FBS and spun down for 5 minutes at 900 RPM. Supernatant is removed and cells are resupsended in Lifeline media containing antibiotics as necessary. Cells are plated onto collagen coated flasks at approximately 4 millions cells per flask and stored at 37C and 5\% CO2. After $24 \mathrm{~h}$ of seeding if there are still floating cells, add $8 \mathrm{~mL}$ of fresh LifeLine media without removing any media. Cells are fed daily with fresh warm LifeLine media. Upon reaching $80 \%$ confluence, cell culture plates are prepared by placing small picces of decellularized tracheal mucosa in the wells. Cells are trypsinized from the flasks, spun down at 900RPM for five minutes and resuspended in LifeLine media. LifeLine media is added to the well and approximately 250,000 cells are added to the apical mucosal surface. Tissue was cultured in an incubator at 37C and 5\% carbon dioxide. Three days later cells were fixed with $10 \%$ neutral buffered formalin for imaging.

\subsubsection{Histology}

Fixed porcine tissue was embedded in parrafin for traditional hemotoxylin and eosin staining of 5 micron sections. Fixed tracheal mucosa was imaged with differential interference contrast microscopy to visualize epithelial coverage and phenotype. To visualize cytoskeletal structure a FITC conjugated phalloidin stain was also employed. Specimens were permeabilized in 0.1\% Triton X-100 in PBS for 1 hour prior to staining with 1:200 dilutions of phalloidin (Molecular Probes, Life Technologies) 


\subsubsection{Statistics}

Statistical comparisons between ultimate load at failure during suture retention was accomplished via t-test. Significance was gained for all tests at $\mathrm{p}<0.05$.

\subsection{RESULTS AND DISCUSSION}

\subsubsection{Cyclical Vacuum Capable of Successful Porcine Tracheal Decellularization}

Cyclic pressure variation was hypothesized to accelerate and improve tissue decellularization through increased tissue perfusion. The removal of mechanical agitation necessary in many of the previous approaches is also believed to contribute to an improvement in the tissue properties. Additional work was conducted employing this technique on a host of other tissues, including aorta, dermis, vocal fold, brain, optic nerve, and urinary bladder matrix. Cyclical pressure was shown to be efficacious for both dense fibrous tissues, as well as delicate

nervous tissue such as brain. Figure 4.9 demonstrates hematoxylin and eosin staining of decellularized porcine trachea processed with the device. Protocol was capable of cellular removal from the mucosa and intercartilaginous segments as with rodent trachea. However, as before, some residual nucelar material was maintained within the chondrocyte lacunae.

\subsubsection{Xenogeneic Scaffold is Mechanically Suitable for Transplant}

Suture retention testing demonstrated little to no difference in load to failure or stiffness of decellularized and native porcine trachea. Interestingly there was a pronounced toe region within the uniaxial data suggesting a loosening of the fibrillar structure which may in turn delay full fiber recruitment (Figure 4.10). However, it is not expected that this effect will alter the physiological function in any way. Additionally, pressure diameter testing at both highly supra- and sub-physiologic pressures demonstrated a safety factor $>3$. 


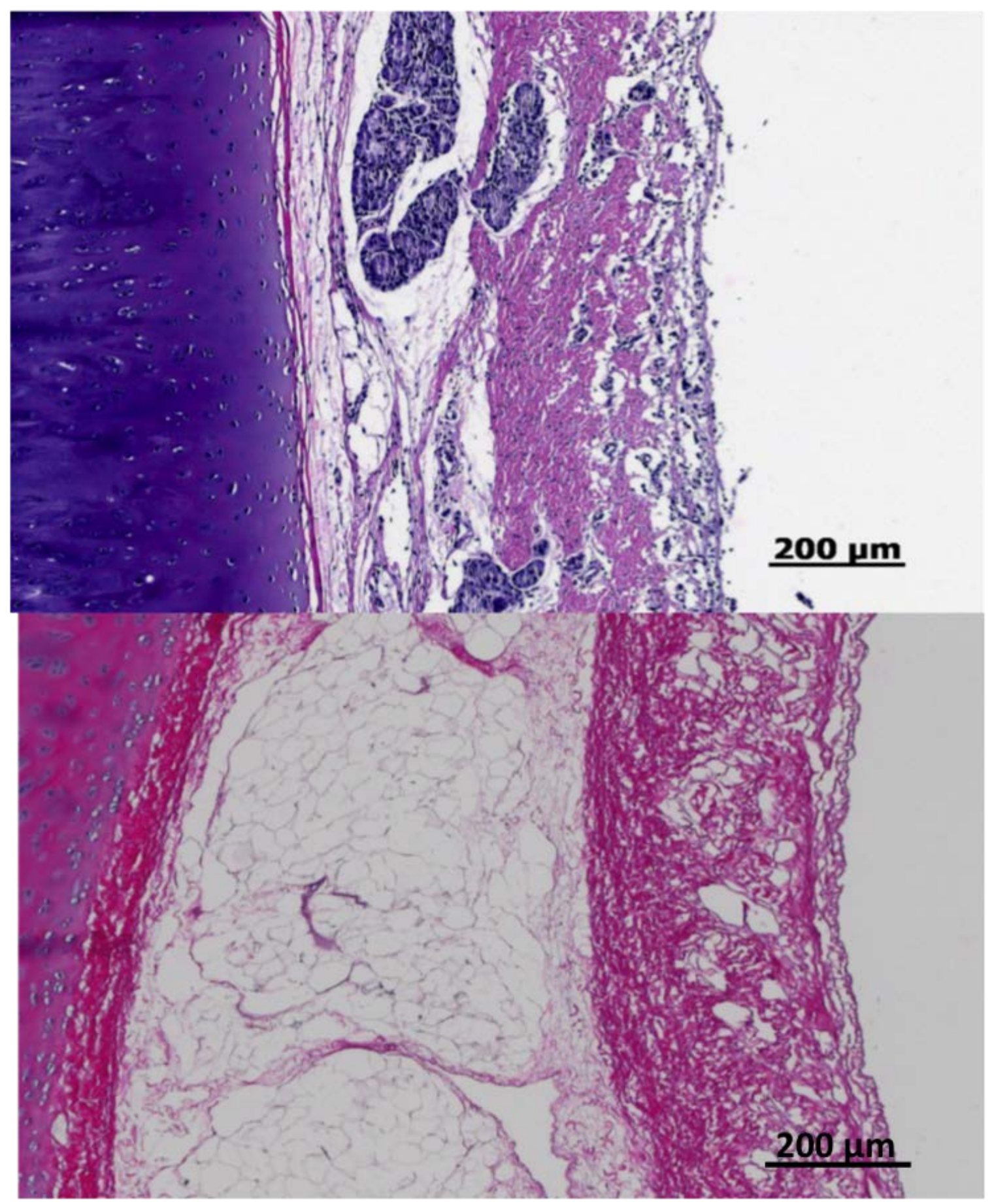

Figure 4.9: Hemotoxylin \& Eosin staining of native (top) and vacuum decellularized (bottom) porcine trachea 

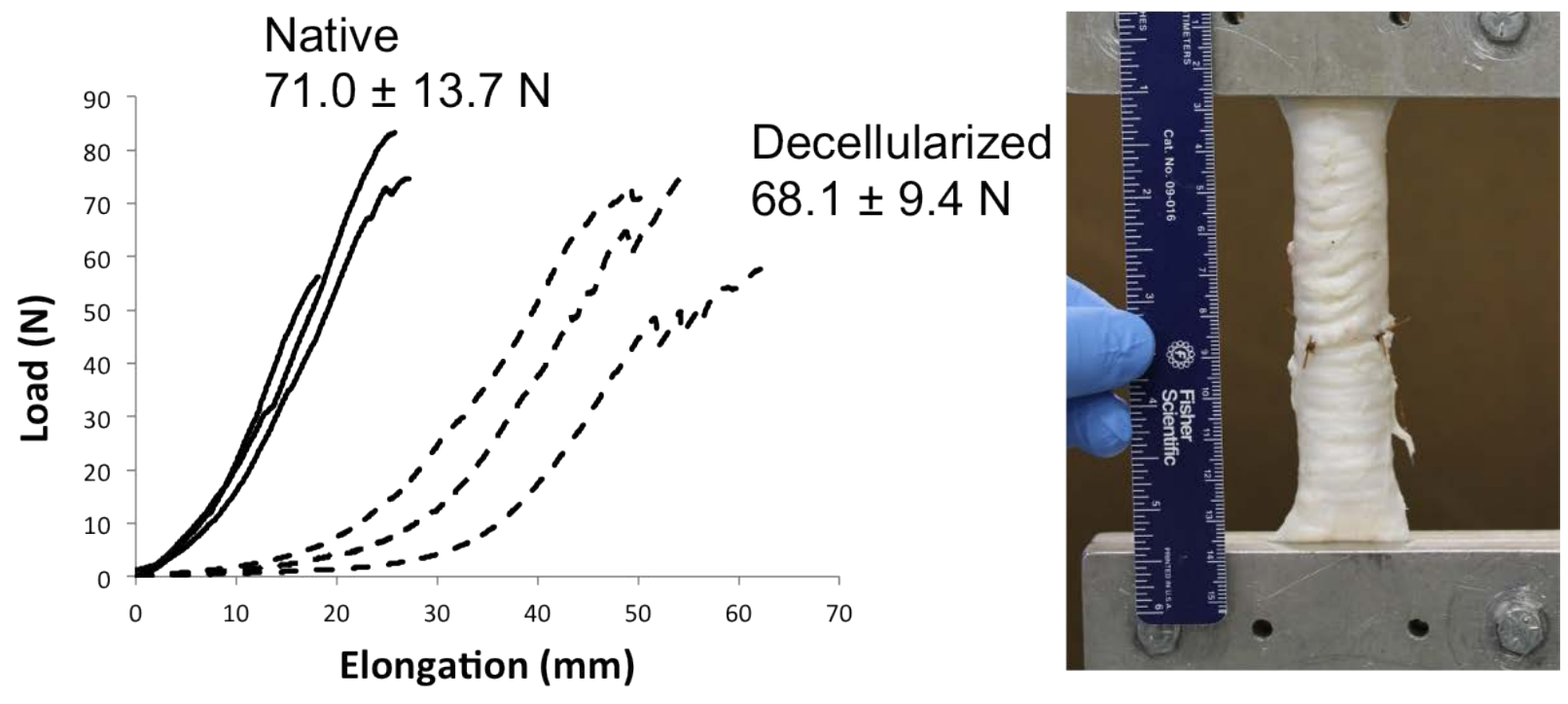

Figure 4.10: Left: Suture retention curves showing load elongation. Mean and standard deviation for each group is presented. Right: Suture retention setup showing a reanastomosed trachea clamped prior to uniaxial load to failure testing on a Instron 4502 


\subsubsection{Porcine Scaffolds Support Healthy Human Respiratory Epithelium}

After 72 hours in vitro human bronchial epithelial cells showed good attachment with excellent coverage (Figure 4.11). A control specimen for the cells was also seeded onto a transwell filter for full air liquid interface culture to demonstrate the ability of this particular patient's epithelial cells to fully differentiate. Results demonstrated full differentiation with cilia production, as evaluated by phase contrast microscopy.

\subsubsection{Limitations and Future Work}

As discussed, one of the main concerns with the translation to a large scaffold is the necessity of quick vascular restoration upon implantation. The grafts to date have survived with a combination of omental wrapping and in vitro seeding of bone marrow derived mesenchymal stem cells. Autologous MSC retrieval is mostly non-invasive and likely necessary to maintain cartilage structure and function in a larger implant. Additionally, MSC seeding is relatively straight forward and would not require complex rotating air liquid culture. To address concerns with reepithelialization over a longer implant length, we propose an intraoperative epithelial seeding process as first tested clinically by Macchiarini et al. to simplify the preculture process and expedite implantation[117, 148]. Given the dramatic improvement seen with Triton X-100 and PAA treatment in a rodent model, epithelial seeding may eventually be found to be unnecessary.

\subsection{CONCLUSIONS}

Decellularization with Triton X-100 and PAA was identified as the optimum procedure in specific aim 2, however, due mostly to size differences, direct translation to a porcine tissue was not possible. Therefore, a cyclical pressure device was developed and employed. The resultant decellularization process was efficacious, demonstrating cellular removal from the mucosa and intercartilaginous segments. Additionally, the cyclical pressure approach obviated the need for long treatment times with harsh detergents, our 14 day protocol is nearly 

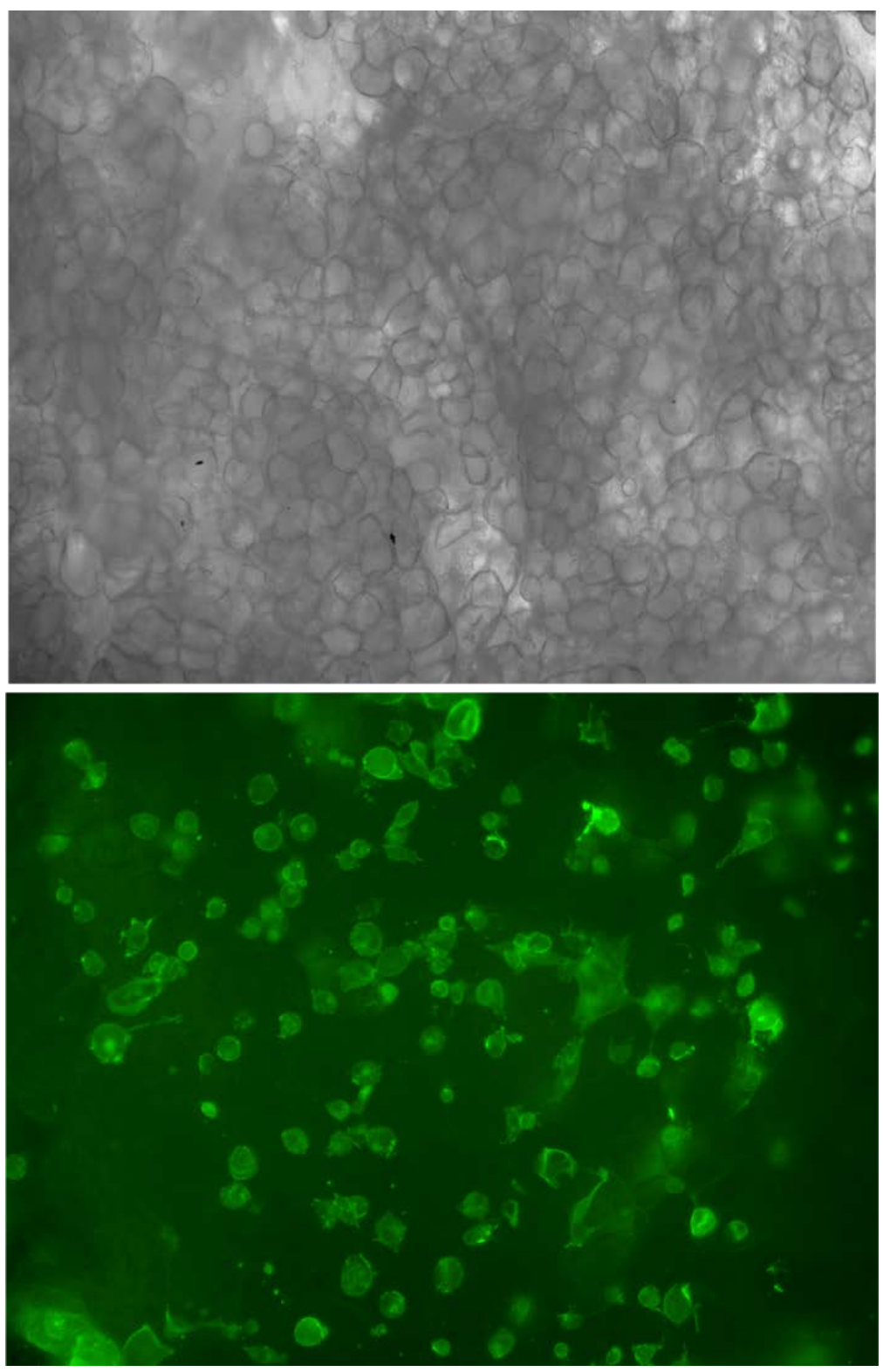

Figure 4.11: Histology of fixed tracheal mucosa after 72 hours seeding in vitro with human bronchial epithelial cells. Top: Differential interference microscopy stack showing confluent epithelium. Bottom: FITC conjugated phalloidin stained epithelial cells 
$300 \%$ faster than existing clinical protocols. The use of decellularized xenografts is of further benefit when the ability to stock pre-sized grafts eliminates the need for lengthy graft preparations for each patient. However, most importantly, our development of a xenogeneic graft obviates the need for any of the critically needed human donor tissue. It is hoped that after suitable large animal implants we may eventually progress towards a first in human trial.

\subsection{ACKNOWLEDGMENTS}

We'd like to thank William Barone, Michelle Weaver, and Dr. Steven Abramowitch for their help with all of the mechanical testing modalities described above. We'd also like to thank Stefanie Brown and Dr. Myerburg for their assistance with HBEC culture and air liquid interface controls. 


\subsection{SUMMARY AND CONCLUSIONS}

Patients diagnosed with primary tracheal cancers were historically palliated in the absence of any effective treatment options. Thankfully, landmark studies from European clinicians have demonstrated success in limited trials of decellularized human trachea grafts [148]. These ECM implants offer a wonderful opportunity for patients with no other viable options. However, there are significant barriers to more widespread implementation of this life saving technology. Current standards employ harsh ionic detergents in a long, drawn out process. Sodium deoxycholate, the most commonly employed detergent, is known to denature proteins both in vitro and in vivo [180, 181, 182, 183, 184]. More than simply removing cellular antigens and debris, ECM grafts processed in this way may not retain the growth factors, proteoglycans, and other matrix proteins in a native configuration capable of providing cues to infiltrating host cells, potentially degrading performance and outcomes. In addition to the harsh processing there are prohibitive preparation times and dire supply limitations associated with human donor tissue. In this study we have sought to evaluate a number of approaches to develop a clinically viable xenogeneic tracheal graft. It was hoped that the use of such a xenograft would eliminate many of the challenges associated with the current generation of devices.

\subsection{NOVEL FINDINGS AND FUTURE DIRECTIONS}

Through improved pre-implant characterization, empirical testing in small animal models, and translation to a porcine derived xenogeneic scaffold using a novel decellularization approach, we have demonstrated that improvements to existing prosthetics are eminently at- 
tainable with minor modifications to well accepted existing protocols. While further work is obviously necessary to prove efficacy in a large animal model prior to human trials, we believe that this work has demonstrated that a clinical xenograft is certainly realistic and potentially attainable. The following synopsis is a concise summary of the notable findings.

\subsubsection{Structural and Compositional Characterization of Decellularized Scaffolds is Vital to Tissue Engineering Success}

When employed for tissue engineering, extracellular matrix (ECM) scaffolds are desirable for many reasons. They are readily repopulated by host cells, degraded in vivo and replaced with de novo tissue deposited by the invading host cell population. Unlike polymeric scaffolds, they also contain remnant proteins and signaling molecules, which can provide very potent cues for regeneration. These decellularized matrices may be obtained through treatment with detergents, acids/bases, enzymes, alcohol, or even mechanical disruption. Unfortunately, there is no universal protocol so the most effective decellularization agents must be identified for each tissue due to the specificity of structure, composition and geometry. It is generally accepted that no tissue may be decellularized without disruption of the native structure. Therefore, the processing conditions and disruptions which drive negative functional outcomes must be identified and avoided. Unfortunately, the clinically employed protocol, the detergent enzymatic method (DEM) was not generated based on a rational and structured approach. Rather, they have simply employed the first protocol which appeared to work well-enough in large animal testing. Sadly, this is in line with much of the literature. A variety of detergents have been widely employed for decellularization of cartilaginous tissues and a number of these studies have shown histologic retention of native-like microstructure [194, 195]. However, few studies employ head-to-head comparisons in the same animal model under controlled circumstances. Therefore, we took a step back and expanded the process design space in the hopes of identifying a more efficacious approach, or at least correlating a wider range of scaffold parameters to functional outcomes.

In addition to the sodium deoxycholate employed in the DEM, we evaluated three alternate, commonly employed detergents: CHAPS, Triton X-100, and SDS. We also tested per- 
acetic acid (PAA), since previous work had shown that PAA effectively removes cells and remnant DNA while having a minimal effect on ECM composition and structure [185, 186, 187]. It was found after processing that all groups were capable of successful decellularization, effectively maintaining organ shape and structure. Unfortunately, traditional metrics employed to assess decellularization were ineffective. H\&E staining between groups was highly similar and both DNA content and fragment length were virtually identical. Other histological stains (Movat's, etc.) were basically indistinguishable, showing only minor differences in some protein levels. Traditionally employed biochemical assays (Blyscan, etc.) that specifically measure ECM constituents were found to be imprecise due to their complex user input requirements. These results all suggested that more complex analysis was required. To this end we employed differential scanning calorimetry (DSC), a sensitive technique whereby differences in the amount of enthalpy required to increase the temperature of a sample compared to a reference is measured as a function of temperature. It allowed us to probe the thermal stability of proteins within an extracellular matrix scaffold. Various components within a scaffold have remarkably different denaturation temperatures, thus DSC can be used to not only assess compositional changes, but also differences in protein cross linking, hydrogen bonding, and hydration states. Previously, probing these questions required detailed transmission electron microscopy (TEM) studies. As with any electron microscopy technique, TEM require extensive sample processing, which may result in structural artifacts. The application of DSC to our tracheal scaffolds enabled us to assess micro-structural and compositional changes with each step of the decellularization process in a bulk, high throughput manner.

The DSC results demonstrated enormous differences in scaffolds which had previously seemed remarkably similar. SDS was shown to completely denature the higher order collagen structures in both the fibrous and cartilaginous portion of the trachea, while Triton X-100 and CHAPS demonstrated preservation of mature collagen cross-linking and matrixassociated proteins. Sodium deoxycholate treatment demonstrated partial denaturation, somewhere between SDS and Triton X-100. This is to be expected given it's relative strength compared to these detergents. Through DSC analysis it also became clear that PAA treatment has a much more marked effect than originally anticipated. This contradicts existing 
literature $[185,186,187]$ and is yet another testament to the importance of increasingly sophisticated analysis techniques, especially those capable of bulk tissue analysis. Through quantification of total enthalpy, a measure of overall thermal stability and higher order structure, we were able to determine that PAA significantly reduces thermal stability compared to all non-PAA groups. Additionally, SDS was found to be significantly different than all of the non-PAA treated specimens.

Further work is necessary to contextualize these DSC findings and identify the classes of proteins and other structural elements that may be retained or removed. Proteomic analysis is an ideal tool to investigate this question and is a logical next step. The ability to cross reference a database of retained proteins with a specific DSC curve would be enormously valuable moving forward. In conjunction with targeted immunofluorescent stains it may be possible to build a model employing informatics techniques to predict host remodeling in a specific tissue. We also recognize that filtration and characterization of discarded decellularization solutions may be a tractable approach to investigate the time-varying removal of protein during processing. In conjunction with experiments assessing the bioactivity of retained growth factors, especially those correlated with cell survival and proliferation in vitro, we could develop a combined dataset which would allow an increasingly holistic study of the scaffold.

\subsubsection{Processing Conditions Predetermines Host Mediated Remodeling}

It is well understood that upon ECM implantation, host macrophages begin immediately infiltrating the scaffold. Recent studies have shown that these macrophages are potent modulators of disease and tissue remodeling following injury. M1 and M2 macrophages have also been shown to play distinct roles in tissue remodeling following injury and differential macrophage phenotypes may catalyze either positive or negative tissue remodeling. Effectively, the biochemical and structural characteristics of the implanted device influence the host macrophages, altering their polarization profile and predetermine the host response. In Chapter 3 we observed this exact effect through the remarkable differences in host survival and remodeling outcomes among the various treatment groups. Animal survival was 
strongly correlated with treatment regimen. Results demonstrated increased survival trends (non-significant) of Triton X-100 and SDS groups compared to sodium deoxycholate. Oddly, CHAPS decellularized grafts performed significantly worse than any of the other three nonPAA treated groups. Counter to our original hypothesis, the PAA treatment groups demonstrated no deaths, indicating similar performance to the best untreated grafts. Perhaps the most interesting result is that the simple addition of a PAA rinse significantly improves the survival of sodium deoxycholate recipient animals at the 12 week time point. Given the histological finding that mucous obstruction was the most likely cause of death, our initial supposition that acute inflammation is mediated by an infiltrating macrophage population is highly probable. For animals surviving to end point, chronic inflammation and poor host response was more prevalent among those specimens with increased mortality. While all surviving implanted rodents had patent grafts with intact and mechanically sound cartilage, stenosis was readily apparent in many of the CHAPS and sodium deoxycholate specimens. SDS, which had demonstrated the greatest level of scaffold disruption, had excellent survival and minimal stenosis, but fibrotic strictures were observed luminally between the cartilage rings. PAA treatment seems to dramatically improve the gross morphology with decreased stricture formation and stenosis, which we believe is predictive of longer term in vivo performance.

From the review of clinical and experimental literature, lack of revascularization seems to be the largest hurdle for tracheal reconstruction and replacement. Many earlier studies demonstrated that insufficient revascularization was a major obstacle to successful remodeling in living tissue transplantation. Further attempts demonstrated that vascularized grafts were much less likely to necrose than free patches $[90,91,92,93,10]$. It is possible that the necrosis resulting from non-vascularized cells causes a chronic inflammatory response that drives the negative surgical outcomes in these historic studies. While not directly applicable, the concurrent restoration of a viable vascular supply after implant is vital to the success of a tissue engineered graft. From the gross histology we were able to observe that PAA treated specimens demonstrate a healthy, native appearance with distinct vasculature. Overall, it appears that PAA is strongly correlated with revascularization and tissue integration, while CHAPS treatment is highly predictive of poor performance. One significant limitation of the 
current study is that we are unable to specifically determine the mechanisms underpinning these findings. However, there are several potential mechanisms which may be responsible for the notable improvement. Since we know that CHAPS is the weakest detergent with the smallest micelle, it may simply have been incapable of properly solubilizing and removing larger membrane-bound proteins after cell lysis. It's known that membrane-bound proteins are largely responsible for cell-to-cell recognition, and their retention may have triggered a more potent inflammatory response. This stronger response might have subsequently contributed to the lack of revascularization, eventually leading to stenosis and necrosis.

PAA, on the other hand, has been shown in some studies to aid in removal of remnant detergent and enzymes [208], which may substantially improve the host cellular response. This would potentially explain the improvement in sodium deoxycholate, but since we did not test CHAPS and SDS with a PAA rinse we cannot confirm this supposition. This is a logical follow-up experiment. Second, PAA treatment has been shown to increase porosity in a number of dense collagenous tissues, including the tendon [209], which could improve cellular infiltration and subsequently increase angiogenesis. This supposition could be readily examined by mid-point analysis to assess the rate of revascularization. Finally, it is possible that PAA may have acted to partially eliminate endotoxins that may have resulted from bacterial growth during processing. It has been suggested that endotoxins present within the ECM may also affect signalling through the TLR4 pathway, resulting in a pro-inflammatory response [210]. To test this hypothesis, we could simply assess endotoxin levels before and

after the PAA processing step. Other potential avenues of research might yield further information which would allow us to better interpret these results. For example, differential macrophage staining may be employed to further investigate the host immune response [205, 206, 207].

\subsubsection{Peracetic Acid (PAA) Treated Grafts Regenerate a Functional Immuno- logical Barrier}

In addition to its vital mechanical function, the trachea also acts as the first immunological barrier to environmental pathogens. Therefore, to quantify the success of our implants, we 
chose to assess their function as an immunological barrier. Unfortunately, current techniques used to study airway cilia motility are limited. Brush biopsies and simple transverse airway sections are utilized, but these techniques are not viable in rodent specimens due to excessive damage upon removal. Other groups employ various cell culture techniques to obtain sheets of ciliated epithelia from harvested airway cells, but the relevance to in vivo performance is questionable. Real-time DIC imaging allows quick and simple determination of airway cilia function in a large number of different treatment groups. While this is an in vitro technique the airway epithelium remains attached to its supporting tissues which closely mimics its natural in vivo tissue environment. It is important to note that while many studies have been conducted to assess cilia development and function in native and mutant rodent species, no studies to date have shown restoration of functional cilia in a tissue engineered graft. Therefore, the findings presented in Chapter 3 demonstrating regeneration of a fully mature and differentiated epithelium with restoration of native cilia function are unprecedented.

In summary, it was found that the non-PAA treated grafts, excepting Triton X-100, demonstrated sparse ciliation with little to no function. In these specimens, the patchy regions of motile cilia in SDS and sodium deoxycholate grafts were insufficient for analysis. Interestingly, Triton X-100 grafts showed well-distributed functional cilia, but had frequency values that were significantly depressed compared to naive controls. Remarkably, both Triton X-100 and sodium deoxycholate grafts treated with PAA showed ciliary beat frequency comparable to both flanking native sections and naive controls. It is important to note that among the PAA specimens there was also substantial beat coordination and uniformity of beat direction. Since we believe that restoration of cilia function, in terms of both frequency and coordination, is vital to long term graft survival, more in-depth analysis should be undertaken. For example, particle image velocimetry (PIV) is a novel technique in this application. In this approach, tracer particles are dispersed within the fluid and their motion is correlated to fluid flow characteristics. Since fluorescent particles may be employed within our existing setup, we could utilize lower magnification videos to gain longer range data on flow patterns and velocity. We believe this global orientation and fluid flow would be highly predictive of in vivo function and might correlate to the number of re-operations in the mid-term for clinical implants. The lack of functional data accessible through this technique 
and others in the pre-clinical literature is surprising, but simply mirrors the lack of rigor demonstrated by studies culminating in clinical applications. We believe that continuing to employ functional metrics is vital to progressing the field.

Overall, we have made great strides in bringing more complex and sophisticated approaches to processing, scaffold analysis, and explant characterization. The head-to-head comparison of multiple treatment groups coupled with functional analysis is a major step forward. Nevertheless, more work is required to develop a more mechanistic understanding of our findings. It was hoped that immunofluorescent (IF) and histological analysis would be possible to probe the relative prevalence of ciliated epithelium and goblet mucosal cells to better establish the presence of all lineages of differentiated epithelium. However, once it became clear that the functional analysis effectively prevented further histological analysis it was determined that observing and quantifying ciliary beat frequency was more vital to overall study goals. It is entirely possible that alteration or removal of basement membrane constituents as a result of decellularization disrupts the cues that assist with proliferation and site-specific differentiation. However, the anomalous improvement in sodium deoxycholate and Triton X-100 after PAA treatment make this argument less straight-forward. Instead, as described above, it may be better explained by removal of residual detergent. Since sodium deoxycholate has a much higher monomer affinity to proteins, it might also explain the differential performance between non-PAA treated Triton X-100 and sodium deoxycholate. Our proposition that PAA-driven increases in porosity leading to improved vascularity may also have a synergistic effect. This would explain the sparser ciliation and decreased cilia function in non-PAA treated specimens. While it may not be possible to decouple revascularization from broader in vivo cytocompatibility, distinguishing the time line of the epithelial and vascular regeneration may be beneficial to establish the temporal sequence of events. This would be best investigated through detailed histological analyses at study mid points, preferably at 4 and 8 weeks. We would also propose supplemental studies evaluating relative proliferation in populations of tracheal basal cells over time [212]. It is known that $\mathrm{K} 5+/ \mathrm{K} 14+$ basal cells represent a precursor cell population with the capacity to develop into ciliated and secretory cells in native trachea. This more detailed approach could shed light on the relationship between the altered scaffold basement membranes seen 
in Chapter 2, differential basal cell proliferation, and eventual epithelial differentiation in vivo.

\subsubsection{Cyclical Pressure Synergistically Improves Detergent Based Decellular- ization}

There has been significant work to characterize the structure and biology of ECM scaffolds and their effect in vivo, yet the chemicals and techniques employed during decellularization have remained relatively unchanged. There have been several novel approaches employing ultrahigh pressure [220] or supercritical carbon dioxide [221]. However, these have shown minimal improvements for the substantial increase in complexity and cost. Other than hypoand hypertonic solutions, traditional treatments do not dramatically improve perfusion into and out of the tissue. Therefore, cyclical hydrostatic pressure was tested to improve infiltration of decellularization chemicals and removal of cellular debris. The efficacy of this approach was presented in three studies prior to translation to the trachea. Two explored the retention of native tissue mechanics during extended decellularization of dermis and vocal fold compared to traditional agitation controls. The third demonstrated the ability of cyclical pressure to fully decellularize aorta and perform successfully in long-term large animal implants. In all three experiments, remarkable results were observed. In vocal fold, dermis, and aorta, decellularization was accomplished extraordinarily quickly under mild processing. All specimens demonstrated significant retention of native mechanics compared to traditional decellualrization controls. For the implanted aortas, the grafts were well integrated with no thrombosis or evidence of a chronic inflammatory response at 12 weeks. Even more importantly, these implanted aortas performed substantially better than cryopreserved specimens, the current clinical gold standard. The summation of these findings gave us the confidence to employ the cyclical pressure approach in translating our rodent findings to porcine tissue. However, due to the obvious size differences among species it may not be possible to directly translate the best protocol from Chapter 3 . Translation to a larger animal may require harsher treatments or longer contact times, potentially negating the improvements produced as a result of optimized processing. It was our hope that the 
cyclical pressure approach would ease this transition given the promising results in other organ systems. Unfortunately, we are unable to specifically address this disjoint given our experimental structure, but in many respects the work conducted in our rodent models is still highly informative. We have been able to conclusively determine the appropriate testing modalities and moving forward will conduct the proposed experiments in the porcine model to ensure direct applicability.

\subsubsection{Xenogeneic Scaffolds are Suitable for Further Preclinical Testing}

As discussed in Chapter 4, the cyclical pressure approach was adopted with little trouble. Notably, the cyclical pressure approach obviated the need for long treatment times and resulted in a 300\% improvement over existing clinical approaches to a 14-day protocol. The resultant decellularization process was efficacious, demonstrating cellular removal from the mucosa and intercartilaginous segments. However, similar to rodent scaffolds there was some residual nuclear material maintained within the chondrocyte lacunae. Given findings that the human tracheal mucosa was the major antigenic structure of tracheal allografts [121], we do not believe this is a major concern.

There is no consensus in the literature regarding the necessity of cellular seeding prior to graft implantation. It is clear that the success of a tracheal graft depends on its structure, mechanics, and antigenicity; however, the cellular component may be relevant. Various groups have attempted to seed a range of cell types, from chondrocytes to mesenchymal stem cells, obtained from a number of donor tissues. As discussed, one of the main concerns with the translation to a large scaffold is the necessity of quick vascular restoration upon implantation. The clinical grafts to date have survived with a combination of omental wrapping and in vitro seeding of bone marrow derived mesenchymal stem cells. Autologous MSC retrieval is mostly non-invasive and may be necessary to maintain cartilage structure and function in a larger implant. However, we believe that with improved processing conditions the need for in vitro seeding may be eliminated.

In the rodent study a relatively short graft was employed and the lack of initial cell seeding may have improved outcomes by temporarily slowing healing; however, the lack of an antigen 
barrier immediately at implant may be a serious concern. Given that there were no infections detected in over 30 rodents, it seems that suitable prophylactic antibiotic administration can significantly hedge this risk. Nevertheless, the progression to a larger graft may require us to develop more sophisticated approaches to quickly restore an immunological barrier after implant. To address these concerns, we propose an intraoperative epithelial seeding process as first tested clinically by Macchiarini et al. to simplify the preculture process and expedite implantation $[117,148]$. If it becomes necessary to employ extended cell seeding, ideally we would obtain a single cell type capable of differentiating into the multiple required cell types. Adipose-derived stem cells [160], bone marrow stromal cells [161, 162, 146, 163, 150, 164], and amniocytes [165] have been utilized for chondrogenesis in the tissue engineered trachea. Cell sources for airway epithelium include respiratory tract endogenous cells and exogenous cells from other tissues in the body (embryonic, bonewalles marrow, amniotic fluid, etc.) [166]. For example, fully differentiated airway epithelium have been generated from embryonic stem cells through an in vitro culture process [167]. Increased research into stem cell technologies such as iPS presents a new avenue for tracheal tissue engineering. However, regulatory challenges will likely postpone their introduction into mainstream use, thus the leading cell source is most likely the patients own bone marrow mesenchymal cells.

In order to de-risk further pre-clinical studies we have employed a myriad of in vitro tests to assess the suitability of a porcine xenograft for eventual human implantation. We know the trachea must operate on several levels simultaneously, as a mechanical tube to support ventilation as well as a front-line immune barrier. Suture retention demonstrated no significant difference in load to failure or stiffness of decellularized and native porcine trachea. A pronounced toe region suggested a loosening of the fibrillar structure which may in turn delay full fiber recruitment. It is not expected that this mechanical feature will alter the physiological function in any way. Additionally, pressure diameter testing at both highly supra- and sub-physiologic pressures demonstrated a safety factor $>3$. The removal of mechanical agitation necessary in many of the previous approaches is believed to have contributed to this improvement in mechanical properties. When considering the immune role, human bronchial epithelial cells were shown to readily attach and proliferate on decellularized porcine tracheal mucosa. This suggests that host cells will be capable of 
infiltrating and recolonizing a porcine graft. Further work is necessary to demonstrate that air-liquid interface culture can drive differentiation of human bronchial epithelial cells to a mature ciliated form.

In conclusion, the use of xenograft material is of enormous benefit. The ability to stock pre-sized grafts eliminates the need for lengthy graft preparations for each patient. More importantly, our development of a xenogeneic graft obviates the need for any of the critically needed human donor tissue. It is hoped that after suitable large animal implants we may test the feasibility of this approach and eventually progress towards a first in human trial.

\subsection{CLOSING REMARKS}

Due to the extraordinarily complex biological milieu, mechanistic studies optimizing a specific decellularization procedure are not yet possible. Not only is the complexity of native extracellular matrices not fully understood, but work is still ongoing to better characterize the decellularization process itself. With our current level of understanding empirical studies

are necessary, but care must be taken to avoid needless experimentation. The tracheal tissue engineering literature is filled with claims of success and failure, but little is learned from each of these experiments since studies often assess a single experimental group. Clearly we cannot claim to have developed a comprehensive design space, but this work represents an incremental improvement in the understanding of tracheal decellularization. In a broader sense, this dissertation aims to address a question inherent to all decellularized tissue engineered products: how does processing influence host remodeling and graft survival? We believe that the techniques and approaches utilized in this study will inform a more rational approach to the design and development of tissue engineered transplants and hope we have laid the groundwork for the success of those efforts. 


\section{BIBLIOGRAPHY}

[1] Z. Thotathil, J. Agarwal, and S. Shrivastava, "Primary malignant tumors of the trachea-the tata memorial hospital experience," Med. Princ. Pract., vol. 13(2), pp. 6973, Jan 2004.

[2] A. L. Pahor, "Ear, nose and throat in ancient egypt," J Laryngol Otol, vol. 106, pp. 773-9, Sep 1992.

[3] E. Frost, "Tracing the tracheostomy," Ann Otol Rhinol Laryngol, vol. 85(5 PT. 1), pp. 618-24, Jan 1976.

[4] R. Belsey, "Resection and reconstruction of the intrathoracic trachea," $B r J$ Surg, vol. 38 , pp. 200-5, Oct 1950.

[5] A. M. Morfit, A. J. Neerken, A. Prevedel, E. B. Liddle, and L. Kircher, "Sleeve resections of the trachea; experimental studies on regenerative capacity and principles of reconstruction and repair," AMA Arch Surg, vol. 70, pp. 654-61, May 1955.

[6] J. Borrie and N. R. Redshaw, "Prosthetic tracheal replacement," J Thorac Cardiovasc Surg, vol. 60, pp. 829-35, Dec 1970.

[7] J. Borrie, N. R. Redshaw, and T. L. Dobbinson, "Silastic tracheal bifurcation prosthesis with subterminal dacron suture cuffs," J Thorac Cardiovasc Surg, vol. 65, pp. 956-62, Jun 1973.

[8] W. E. Neville, J. P. Bolanowski, and G. G. Kotia, "Clinical experience with the silicone tracheal prosthesis," J Thorac Cardiovasc Surg, vol. 99, pp. 604-12; discussion 612-3, Apr 1990.

[9] H. Toomes, G. Mickisch, and I. Vogt-Moykopf, "Experiences with prosthetic reconstruction of the trachea and bifurcation," Thorax, vol. 40, pp. 32-7, Jan 1985.

[10] H. C. Grillo, "Tracheal replacement: a critical review," Ann. Thorac Surg, vol. 73, pp. 1995-2004, Jun 2002.

[11] R. A. Daniel, "The regeneration of defects of the trachea and bronchi; an experimental study," J Thorac Surg, vol. 17, pp. 335-49, Jun 1948. 
[12] T. W. Wykoff, "A preliminary report on segmental tracheal prosthetic replacement in dogs," Laryngoscope, vol. 83, pp. 1072-7, Jul 1973.

[13] E. J. Beattie, B. Blades, and J. M. Keshishian, "Tracheal reconstruction," J Thorac Surg, vol. 32, pp. 707-27, Dec 1956.

[14] E. Michelson, R. Solomon, L. Maun, and J. Ramirez, "Experiments in tracheal reconstruction," J Thorac Cardiovasc Surg, vol. 41, pp. 748-59, Jun 1961.

[15] W. P. Longmire, "The repair of large defects of the trachea," Ann Otol Rhinol Laryngol, vol. 57, pp. 875-83, Sep 1948.

[16] J. H. Grindlay, O. T. Clagett, and J. H. Moersch, "Resection and anastomosis of the trachea; an experimental study," Ann Otol Rhinol Laryngol, vol. 58, pp. 1225-9, Dec 1949.

[17] R. L. Craig, G. W. Holmes, and E. J. Shabart, "Tracheal resection and replacement with a prosthesis," J Thorac Surg, vol. 25, pp. 384-96, Apr 1953.

[18] R. R. Shaw, A. Aslami, and W. R. Webb, "Circumferential replacement of the trachea in experimental animals," Ann Thorac Surg, vol. 5, pp. 30-5, Jan 1968.

[19] A. J. Spinazzola, J. L. Graziano, and W. E. Neville, "Experimental reconstruction of the tracheal carina," J Thorac Cardiovasc Surg, vol. 58, pp. 1-13, Jul 1969.

[20] D. Kramish and H. M. Morfit, "The use of a teflon prosthesis to bridge complete sleeve defects in the human trachea," Am J Surg, vol. 106, pp. 704-8, Nov 1963.

[21] B. J. Bailey and J. Kosoy, "Observations in the development of tracheal prostheses and tracheal transplantation," Laryngoscope, vol. 80, pp. 1553-65, Oct 1970.

[22] V. O. Bjork and L. E. Rodriguez, "Reconstruction of the trachea and its bifurcation; an experimental study," J Thorac Surg, vol. 35, pp. 596-603, May 1958.

[23] W. H. Moncrief and J. E. Salvatore, "An improved tracheal posthesis," Surg Forum, vol. 9, pp. 350-2, Jan 1958.

[24] F. J. Jarvis, "Pulmonary resection in the treatment of pulmonary tuberculosis. a study of 100 consecutive cases," West J Surg Obstet Gynecol, vol. 58, pp. 643-7, Nov 1950.

[25] B. H. Cotton, B. Hills, and J. R. F. Penido, "Resection of the trachea for carcinoma; report of two cases," J Thorac Surg, vol. 24, pp. 231-45, Sep 1952.

[26] O. T. Clagett, H. J. Moersch, and J. H. Grindlay, "Intrathoracic tracheal tumors: development of surgical technics for their removal," Ann Surg, vol. 136, pp. 520-32, Sep 1952. 
[27] J. W. McCall and C. W. Whitaker, "The use of prostheses in the larynx and trachea. a preliminary report," Trans Am Laryngol Assoc, vol. 83, pp. 16-24, Jan 1962.

[28] M. Y. Atamanyuk and D. G. Melrose, "The treatment of circumferential defects of the trachea," Br J Surg, vol. 52, pp. 59-65, Jan 1965.

[29] S. Ekestrom and E. Carlens, "Teflon prosthesis in tracheal defects in man," Acta Chir Scand Suppl, vol. Suppl 245, pp. 71-5, Jan 1959.

[30] F. Pearson, R. Henderson, A. Gross, R. J. Ginsberg, and R. M. Stone, "The reconstruction of circumferential tracheal defects with a porous prosthesis. an experimental and clinical study using heavy marlex mesh.," J Thorac Cardiovasc Surg, vol. 55(5), pp. 605-616, Jan 1968.

[31] K. Moghissi, "Tracheal reconstruction with a prosthesis of marlex mesh and pericardium," J Thorac Cardiovasc Surg, vol. 69, pp. 499-506, Apr 1975.

[32] R. M. Bucher, W. E. Burnett, and G. P. Rosemond, "Experimental reconstruction of tracheal and bronchial defects with stainless steel wire mesh.," Journal Thorac Surg, vol. 21(6), pp. 572-83, Jan 1951.

[33] S. Greenberg and R. Willms, "Tracheal prostheses: an experimental study in dogs," Arch of Otol, Jan 1962.

[34] S. M. Poticha and F. J. Lewis, "Experimental replacement of the trachea," J Thorac Cardiovasc Surg, vol. 52(1), pp. 61-7, Jan 1966.

[35] C. G. Rob and G. H. Bateman, "Reconstruction of the trachea and cervical esophagus preliminary report," Br J Surg, vol. 37(146), pp. 202-5, Jan 1949.

[36] D. Ferguson, J. Wild, and O. Wangensteen, "Experimental resection of the trachea," Surgery, vol. 28(3), pp. 597-619, Jan. 1950.

[37] M. Carter and J. Strieder, "Resection of the trachea and bronchi; and experimental study," J Thorac Surg, vol. 20(4), pp. 613-27, Jan 1950.

[38] E. Swift, J. Grindlay, and O. Clagett, "The repair of tracheal defects with fascia and tantalum mesh: an experimental study.," J Thorac Surg, vol. 24(5), pp. 482-92, Jan 1952.

[39] L. Kiriluk and K. Merendino, "An experimental evaluation in the dog of bronchial transplantation, bronchial. tracheal and tracheobronchial resection with reconstruction," Ann Surg, vol. 137(4), pp. 490-503, Jan 1953.

[40] S. H. Mendak, R. J. Jensik, M. F. Haklin, and D. L. Roseman, "The evaluation of various bioabsorbable materials on the titanium fiber metal tracheal prosthesis," Ann Thorac Surg, vol. 38(5), pp. 488-493, Jan 1984. 
[41] A. C. Beall, O. Harrington, S. Greenberg, M. G. C, and U. F. C, "Tracheal replacement with heavy marlex meshcircumferential replacement of the cervical trachea," Arch Surg, vol. 84(4), pp. 390-96, Jan 1962.

[42] T. Sekine, T. Nakamura, K. Matsumoto, Y. Liu, H. Ueda, N. Tamura, and Y. Shimizu, "Carinal reconstruction with a y-shaped collagen-conjugated prosthesis," J Thorac Cardiovasc Surg, vol. 119(6), pp. 1162-1168, Jan 2000.

[43] N. Okumura, T. Nakamura, T. Natsume, K. Tomihata, and Y. Shmizu, "Experimental study on a new tracheal prosthesis made from collagen-conjugated mesh.," J Thorac Cardiovasc Surg, vol. 108(2), pp. 337-45, Jan 1994.

[44] M. Teramachi, N. Okumura, T. Nakamura, Y. Yamamoto, T. Kiyotani, Y. Takimoto, S. Matsuda, Y. Ikada, and Y. Shimizu, "Intrathoracic tracheal reconstruction with a collagen-conjugated prosthesis: evaluation of the efficacy of omental wrapping," $J$ Thorac Cardiovasc Surg, vol. 113(4), pp. 701-11, Jan 1997.

[45] J. R. Bottema and C. R. H. Wildevuur, "Incorporation of microporous teflon tracheal prostheses in rabbits: evaluation of surgical aspects," J Surg Res, vol. 41(1), pp. 16-23, Jan 1986.

[46] D. Cull, K. Lally, E. Mair, and M. Daidone..., "Tracheal reconstruction with polytetrafluoroethylene graft in dogs," Annals of Thoracic Surgery, vol. 50(6), pp. 899-901, Jan. 1990.

[47] R. Nelson, L. Goldberg, R. White, E. Shors, and F. M. Hirose, "Neovascularity of a tracheal prosthesis/tissue complex.," J Thorac Cardiovasc Surg, vol. 86(6), pp. 800-8, Jan 1983.

[48] R. E. Taber and L. Tomatis, "Experimental and clinical utilization of a prosthesis for replacement of the trachea," Arch. Surg., vol. 77(4), pp. 576-583, Jan 1958.

[49] J. R. Jacobs, "Investigations into tracheal prosthetic reconstruction," Laryngoscope, vol. 98(11), pp. 1239-45, Jan 1988.

[50] W. G. Cahan, "Carcinoma of intrathoracic trachea: excision and repair by tantalum gauze-fascia lata graft; report of a case," J Thorac Surg, vol. 23, pp. 513-27, May 1952.

[51] M. T. Edgerton, "One-stage reconstruction of the cervical esophagus or trachea," Surgery, vol. 31, pp. 239-50, Feb 1952.

[52] T. J. Yeh, G. Batayias, H. Peters, and R. G. Ellison, "Metastatic carcinoma trachea: report of a case of palliation by resection and marlex graft," J Thorac Cardiovasc Surg, vol. 49, pp. 886-92, May 1965.

[53] M. A. Scherer, R. Ascherl, K. Geissdörfer, W. Mang, G. Blümel, H. Lichti, and W. Fraefel, "Experimental bioprosthetic reconstruction of the trachea," Arch Otorhinolaryngol, vol. 243, pp. 215-23, Jan 1986. 
[54] J. J. Pressman and M. B. Simon, "Tracheal stretching and metaplasia of the tracheal rings from cartilage to bone following the use of aortic homografts," Am Surg, vol. 25, pp. 850-6, Nov 1959.

[55] A. G. Marrangoni, "Homotransplantation of tracheal segments preserved by lyophilization; an experimental study," J Thorac Surg, vol. 21, pp. 398-401, Apr 1951.

[56] S. D. Greenberg, "Tracheal reconstruction: an experimental study," Arch Otolaryngol, vol. 72, pp. 565-74, Nov 1960.

[57] J. Bujía, E. Wilmes, J. Bartual-Pastor, and C. Hammer, "Comparative study of the effect of different chemical procedures on the antigenicity of allogenic transplants of the human trachea]," Acta Otorrinolaringol Esp, vol. 44, pp. 209-16, Jan 1993.

[58] J. P. Jacobs, J. A. Quintessenza, T. Andrews, R. P. Burke, Z. Spektor, R. E. Delius, R. J. Smith, M. J. Elliott, and C. Herberhold, "Tracheal allograft reconstruction: the total north american and worldwide pediatric experiences," The Annals of thoracic surgery, vol. 68, pp. 1043-51; discussion 1052, Sep 1999.

[59] J. P. Jacobs, M. J. Elliott, M. P. Haw, C. M. Bailey, and C. Herberhold, "Pediatric tracheal homograft reconstruction: a novel approach to complex tracheal stenoses in children," J Thorac Cardiovasc Surg, vol. 112, pp. 1549-58; discussion 1559-60, Dec 1996.

[60] M. J. Elliott, M. P. Haw, J. P. Jacobs, C. M. Bailey, J. N. Evans, and C. Herberhold, "Tracheal reconstruction in children using cadaveric homograft trachea," Eur $J$ Cardiothorac Surg, vol. 10, pp. 707-12, Jan 1996.

[61] J. S. Davis, "II. The transplantation of free flaps of fascia: An experimental study," Ann Surg, vol. 54, pp. 734-48, Dec 1911.

[62] B. Drettner and C. E. Lindholm, "Experimental tracheal reconstruction with composite graft from nasal septum," Acta Oto-Laryngologica, vol. 70, pp. 401-7, Jan 1970.

[63] L. R. Bryant, "Replacement of tracheobronchial defects with autogenous pericardium," J Thorac Cardiovasc Surg, vol. 48, pp. 733-40, Nov 1964.

[64] E. W. Fonkalsrud and W. G. Plested, "Tracheobronchial reconstruction with autologous periosteum," J Thorac Cardiovasc Surg, vol. 52, pp. 666-74, Nov 1966.

[65] R. C. Cohen, R. M. Filler, K. Konuma, A. Bahoric, G. Kent, and C. Smith, "A new model of tracheal stenosis and its repair with free periosteal grafts," $J$ Thorac Cardiovasc Surg, vol. 92, pp. 296-304, Aug 1986.

[66] B. G. Narodick, L. W. Worman, and A. H. Pemberton, "Relaxation technique for tracheal reconstruction," Ann Thorac Surg, vol. 24, pp. 190-6, Mar 1965. 
[67] F. Eckersberger, E. Moritz, and E. Wolner, "Circumferential tracheal replacement with costal cartilage," J Thorac Cardiovasc Surg, vol. 94, pp. 175-80, Aug 1987.

[68] R. Sato, I. Hasegawa, and J. Nakagawa, "Experimental study of tracheal reconstruction," J Thorac Surg, vol. 34, pp. 526-38, Oct 1957.

[69] L. G. Farkas, A. W. Farmer, W. G. McCain, and W. D. Wilson, "Replacement of a tracheal defect in the dog by a preformed composite graft. a later report," Plast Reconstr Surg, vol. 50, pp. 238-41, Sep 1972.

[70] A. A. Sabás, J. B. Uez, O. Rojas, A. Iñones, and J. A. Aranguren, "Replacement of the trachea with dura mater. experimental work," J Thorac Cardiovasc Surg, vol. 74, pp. 761-5, Nov 1977.

[71] E. E. Cliffton and B. F. Rush, "Experimental reconstruction of the trachea with bladder mucosa," Surgery, vol. 40, pp. 1105-10, Dec 1956.

[72] W. S. Barker and W. B. Litton, "Bladder osteogenesis aids tracheal reconstruction," Arch Otolaryngol, vol. 98, pp. 422-5, Dec 1973.

[73] G. Marshak, J. H. Porter, and A. J. McAdams, "Reconstruction of the canine trachea with urinary bladder wall," Laryngoscope, vol. 83, pp. 1090-5, Jul 1973.

[74] R. E. Jones, R. F. Morgan, K. L. Marcella, S. E. Mills, and I. L. Kron, "Tracheal reconstruction with autogenous jejunal microsurgical transfer," Ann Thorac Surg, vol. 41, pp. 636-8, Jun 1986.

[75] L. Ohlsén and U. Nordin, "Tracheal reconstruction with perichondrial grafts," Scand J Plast Reconstr Surg, vol. 10, pp. 135-45, Jan 1976.

[76] D. L. Paulson, "Plastic reconstruction of trachea and bronchi," Am Rev Tuberc, vol. 64, pp. 477-88, Nov 1951.

[77] P. W. Gebauer, "Further experiences with dermal grafts for healed tuberculous stenosis of the bronchi and trachea," J Thorac Surg, vol. 20, pp. 628-67, Oct 1950.

[78] B. H. Evans, "Myxochondroma of the trachea; a case report," J Thorac Surg, vol. 22, pp. 585-91, Dec 1951.

[79] V. Caputo and V. Consiglio, "The use of patient's own auricular cartilage to repair deficiency of the tracheal wall," J Thorac Cardiovasc Surg, vol. 41, pp. 594-6, May 1961.

[80] S. J. MacHale, "A new technique for repairing the major air passages," J Thorac Cardiovasc Surg, vol. 64, pp. 6-10, Jul 1972. 
[81] A. T. Cheng, C. L. Backer, L. D. Holinger, M. E. Dunham, C. Mavroudis, and F. Gonzalez-Crussi, "Histopathologic changes after pericardial patch tracheoplasty," Arch Otolaryngol Head Neck Surg, vol. 123, pp. 1069-72, Oct 1997.

[82] K. Kimura, N. Mukohara, C. Tsugawa, Y. Matsumoto, C. Sugimura, H. Murata, and H. Itoh, "Tracheoplasty for congenital stenosis of the entire trachea," J Pediatr Surg, vol. 17, pp. 869-71, Dec 1982.

[83] F. S. Idriss, S. Y. DeLeon, M. N. Ilbawi, C. R. Gerson, G. F. Tucker, and L. Holinger, "Tracheoplasty with pericardial patch for extensive tracheal stenosis in infants and children," J Thorac Cardiovasc Surg, vol. 88, pp. 527-36, Oct 1984.

[84] C. L. Backer, C. Mavroudis, M. E. Dunham, and L. D. Holinger, "Reoperation after pericardial patch tracheoplasty," J Pediatr Surg, vol. 32, pp. 1108-11; discussion 11112, Jul 1997.

[85] C. Tsugawa, E. Nishijima, T. Muraji, Y. Matsumoto, M. Yamaguchi, H. Murata, and K. Kimura, "The use of omental pedicle flap for tracheobronchial reconstruction in infants and children," J Pediatr Surg, vol. 26, pp. 762-5, Jul 1991.

[86] E. H. Dykes, A. Bahoric, C. Smith, G. Kent, and R. M. Filler, "Reduced tracheal growth after reconstruction with pericardium," J Pediatr Surg, vol. 25, pp. 25-9, Jan 1990.

[87] E. Blair, "Study of the viable intercoastal pedicle graft in tracheobronchial surgery," $J$ Thorac Surg, vol. 36, pp. 869-78, Dec 1958.

[88] B. F. Akl, J. Mittelman, D. E. Smith, and C. Butler, "A new method of tracheal reconstruction," Ann Thorac Surg, vol. 36(3), pp. 265-9, Sep 1983.

[89] J. Watson, P. J. Donald, I. M. Gourley, R. Pool, and W. Tyler, "Composite vascularized free-rib and pleural transfer for laryngotracheal reconstruction," Otolaryngol Head Neck Surg, vol. 91, pp. 384-95, Aug 1983.

[90] R. Penton and O. Brantigan, "The use of a viable pedicle graft for repairing an extensive tracheobronchial defect," Ann Surg, vol. 135(5), pp. 709-714, Jan 1952.

[91] B. G. Narodick, A. H. Pemberton, and L. W. Worman, "Tracheoplasty by means of a periosteal pedicle graft," J Thorac Cardiovasc Surg, vol. 47, pp. 572-6, Jan 1964.

[92] F. G. Kergin, "Carcinoma of the trachea," J Thorac Surg, vol. 23, pp. 164-8, Feb 1952.

[93] S. Westaby, M. P. Shepherd, and H. C. Nohl-Oser, "The use of diaphragmatic pedicle grafts for reconstructive procedures in the esophagus and tracheobronchial tree," Ann Thorac Surg, vol. 33, pp. 486-90, May 1982.

[94] J. J. Conley, "Rehabilitation of the airway system by neck flaps," Trans Am Laryngol Assoc, vol. 83, pp. 25-33, Jan 1962. 
[95] D. P. Bryce and V. G. Lawson, "The "trough" method of laryngotracheal reconstruction," Ann Otol Rhinol Laryngol, vol. 76, pp. 793-803, Oct 1967.

[96] T. R. Kapur, "Tracheal injury and reconstruction," Br J Surg, vol. 54, pp. 689-94, Aug 1967.

[97] W. F. Keim, "Total reconstruction of the cervical trachea," Arch Otolaryngol, vol. 91, pp. 501-4, Jun 1970.

[98] G. T. Williams, "New material for a prosthesis used in tracheal reconstruction," $J$ Laryngol Otol, vol. 88, pp. 1175-84, Dec 1974.

[99] W. H. Friedman, H. F. Biller, and M. L. Som, "Repair of extended laryngotracheal stenosis," Arch Otolaryngol, vol. 101, pp. 152-6, Mar 1975.

[100] W. W. ontgomery, "Reconstruction of the cervical trachea," Ann Otol Rhinol Laryngol, vol. 73, pp. 1-15, Mar 1964.

[101] H. F. Biller, W. Lawson, and V. Weisberg, "Staged repair of extensive tracheal and laryngotracheal stenoses," Ann Otol Rhinol Laryngol, vol. 95, pp. 586-9, Jan 1986.

[102] I. Eliachar, M. Goldsher, A. R. Moscona, and D. J. Hurwitz, "Reconstruction of the larynx and cervical trachea with the pectoralis major myocutaneous island flap," Head Neck Surg, vol. 6, pp. 880-3, Jan 1984.

[103] A. Serrano, F. Ortiz-Monasterio, and J. Andrade-Pradillo, "Reconstruction of the cervical trachea. reconstruction of the cervical trachea. a technique to obtain a permanently patent airway," Plast Reconstr Surg Transplant Bull, vol. 24, pp. 333-40, Oct 1959.

[104] C. Papp, J. B. McCraw, and P. G. Arnold, "Experimental reconstruction of the trachea with autogenous materials," J Thorac Cardiovasc Surg, vol. 90, pp. 13-20, Jul 1985.

[105] Y. P. Krespi, H. F. Biller, and S. M. Baek, "Tracheal reconstruction with a pleuroperiosteal flap," Otolaryngol Head Neck Surg, vol. 91, pp. 610-4, Dec 1983.

[106] M. Kon and A. van den Hooff, "Cartilage tube formation by perichondrium: a new concept for tracheal reconstruction," Plast Reconstr Surg, vol. 72, pp. 791-7, Dec 1983.

[107] R. Kato, A. S. Onuki, M. Watanabe, T. Hashizume, M. Kawamura, K. Kikuchi, K. Kobayashi, and T. Ishihara, "Tracheal reconstruction by esophageal interposition: an experimental study," Ann Thorac Surg, vol. 49, pp. 951-4, Jun 1990.

[108] E. Letang, J. Sánchez-Lloret, J. M. Gimferrer, J. Ramírez, and A. Vicens, "Experimental reconstruction of the canine trachea with a free revascularized small bowel graft," Ann Thorac Surg, vol. 49, pp. 955-8, Jun 1990. 
[109] D. E. Strandness, I. J. Gustafson, and J. T. Payne, "Surgical resection of the thoracic trachea: an experimental study in dogs," J Thorac Surg, vol. 34, pp. 269-77, Aug 1957.

[110] W. E. Neville, P. J. Bolanowski, and H. Soltanzadeh, "Homograft replacement of the trachea using immunosuppression," J Thorac Cardiovasc Surg, vol. 72, pp. 596-601, Oct 1976.

[111] R. Nakanishi, T. Shirakusa, and T. Takachi, "Omentopexy for tracheal autografts," Ann Thorac Surg, vol. 57, pp. 841-5, Apr 1994.

[112] R. Nakanishi, T. Shirakusa, and T. Mitsudomi, "Maximum length of tracheal autografts in dogs," J Thorac Cardiovasc Surg, vol. 106, pp. 1081-7, Dec 1993.

[113] S. C. Balderman and G. Weinblatt, "Tracheal autograft revascularization," J Thorac Cardiovasc Surg, vol. 94, pp. 434-41, Sep 1987.

[114] J. F. Khalil-Marzouk, "Allograft replacement of the trachea. experimental synchronous revascularization of composite thyrotracheal transplant," J Thorac Cardiovasc Surg, vol. 105, pp. 242-6, Feb 1993.

[115] P. Macchiarini, B. Lenot, V. de Montpreville, E. Dulmet, G. M. Mazmanian, M. Fattal, F. Guiard, A. Chapelier, and P. Dartevelle, "Heterotopic pig model for direct revascularization and venous drainage of tracheal allografts. paris-sud university lung transplantation group," J Thorac Cardiovasc Surg, vol. 108, pp. 1066-75, Dec 1994.

[116] P. Macchiarini, G. M. Mazmanian, V. T. de Montpréville, E. M. Dulmet, A. L. Chapelier, and P. G. Dartevelle, "Maximal preservation time of tracheal allografts. the parissud university lung transplantation group," The Annals of thoracic surgery, vol. 60, pp. 1597-604, Dec 1995.

[117] P. Macchiarini, G. M. Mazmanian, V. de Montpréville, E. Dulmet, M. Fattal, B. Lenot, A. Chapelier, and P. Dartevelle, "Experimental tracheal and tracheoesophageal allotransplantation. paris-sud university lung transplantation group," J Thorac Cardiovasc Surg, vol. 110, pp. 1037-46, Oct 1995.

[118] M. Strome, J. Stein, R. Esclamado, D. Hicks, R. R. Lorenz, W. Braun, R. Yetman, I. Eliachar, and J. Mayes, "Laryngeal transplantation and 40-month follow-up," N Engl J Med, vol. 344, pp. 1676-9, May 2001.

[119] C. R. Pacheco, O. Rivero, and J. K. Porter, "Experimental reconstructive surgery of the trachea," J Thorac Surg, vol. 27, pp. 554-64, Jun 1954.

[120] A. Beigel, R. Steffens-Knutzen, B. Müller, U. Schumacher, and H. Stein, "Tracheal transplantation. iii. demonstration of transplantation antigens on the tracheal mucosa of inbred rat strains," Arch Otorhinolaryngol, vol. 241, pp. 1-8, Jan 1984. 
[121] J. Bujia, E. Wilmes, C. Hammer, and E. Kastenbauer, "Tracheal transplantation: demonstration of hla class ii subregion gene products on human trachea," Acta Otolaryngol, vol. 110, pp. 149-54, Jan 1990.

[122] T. H. Kalb, M. T. Chuang, Z. Marom, and L. Mayer, "Evidence for accessory cell function by class ii mhc antigen-expressing airway epithelial cells," Am J Respir Cell Mol Biol, vol. 4, pp. 320-9, Apr 1991.

[123] K. G. Rose, K. Sesterhenn, and F. Wustrow, "Tracheal allotransplantation in man," Lancet, vol. 1, p. 433, Feb 1979.

[124] T. Tojo, K. Niwaya, N. Sawabata, K. Kushibe, K. Nezu, S. Taniguchi, and S. Kitamura, "Tracheal replacement with cryopreserved tracheal allograft: experiment in dogs," Ann Thorac Surg, vol. 66, pp. 209-13, Jul 1998.

[125] T. Mukaida, N. Shimizu, M. Aoe, A. Andou, H. Date, K. Okabe, M. Yamashita, and S. Ichiba, "Experimental study of tracheal allotransplantation with cryopreserved grafts," J Thorac Cardiovasc Surg, vol. 116, pp. 262-6, Aug 1998.

[126] H. Yokomise, K. Inui, H. Wada, T. Goh, K. Yagi, S. Hitomi, and M. Takahashi, "Highdose irradiation prevents rejection of canine tracheal allografts," $J$ Thorac Cardiovasc Surg, vol. 107, pp. 1391-7, Jun 1994.

[127] S. Moriyama, N. Shimizu, and S. Teramoto, "Experimental tracheal allotransplantration using omentopexy," Transplant Proc, vol. 21, pp. 2596-600, Feb 1989.

[128] Y. Liu, T. Nakamura, Y. Yamamoto, K. Matsumoto, T. Sekine, H. Ueda, and Y. Shimizu, "Immunosuppressant-free allotransplantation of the trachea: the antigenicity of tracheal grafts can be reduced by removing the epithelium and mixed glands from the graft by detergent treatment," J Thorac Cardiovasc Surg, vol. 120, pp. 108-14, Jul 2000 .

[129] H. Yokomise, K. Inui, H. Wada, M. Ueda, and S. Hitomi, "Long-term cryopreservation can prevent rejection of canine tracheal allografts with preservation of graft viability," J Thorac Cardiovasc Surg, vol. 111, pp. 930-4, May 1996.

[130] T. Mukaida, N. Shimizu, M. Aoe, A. Andou, H. Date, and S. Moriyama, "Origin of regenerated epithelium in cryopreserved tracheal allotransplantation," Ann Thorac Surg, vol. 66, pp. 205-8, Jul 1998.

[131] H. Moriyama, T. Sasajima, S. Hirata, K. Yamazaki, E. Yatsuyanagi, and Y. Kubo, "Revascularization of canine cryopreserved tracheal allografts," The Annals of thoracic surgery, vol. 69, pp. 1701-6, Jun 2000.

[132] T. Tojo, S. Kitamura, S. Gojo, K. Kushibe, K. Nezu, and S. Taniguchi, "Epithelial regeneration and preservation of tracheal cartilage after tracheal replacement with 
cryopreserved allograft in the rat," J Thorac Cardiovasc Surg, vol. 116, pp. 624-7, Oct 1998.

[133] K. Inutsuka, K. Kawahara, T. Takachi, K. Okabayashi, T. Shiraishi, and T. Shirakusa, "Reconstruction of trachea and carina with immediate or cryopreserved allografts in dogs," The Annals of thoracic surgery, vol. 62, pp. 1480-4, Nov 1996.

[134] R. Langer and J. P. Vacanti, "Tissue engineering," Science, vol. 260, pp. 920-6, May 1993.

[135] J. F. Grimmer, C. B. Gunnlaugsson, E. Alsberg, H. S. Murphy, H. J. Kong, D. J. Mooney, and R. A. Weatherly, "Tracheal reconstruction using tissue-engineered cartilage," Arch Otolaryngol Head Neck Surg, vol. 130, pp. 1191-6, Oct 2004.

[136] K. Kojima, L. J. Bonassar, A. K. Roy, C. A. Vacanti, and J. Cortiella, "Autologous tissue-engineered trachea with sheep nasal chondrocytes," J Thorac Cardiovasc Surg, vol. 123, pp. 1177-84, Jun 2002.

[137] C. Vacanti, K. Paige, W. Kim, and J. Sakata..., "Experimental tracheal replacement using tissue-engineered cartilage," J Pediatr Surg, Jan 1994.

[138] X. Luo, G. Zhou, W. Liu, W. J. Zhang, L. Cen, L. Cui, and Y. Cao, "In vitro precultivation alleviates post-implantation inflammation and enhances development of tissueengineered tubular cartilage," Biomed Mater, vol. 4, p. 025006, Apr 2009.

[139] L. Yang, S. Korom, M. Welti, S. P. Hoerstrup, G. Zünd, F. J. Jung, P. Neuenschwander, and W. Weder, "Tissue engineered cartilage generated from human trachea using degrapol scaffold," Eur J Cardiothorac Surg, vol. 24, pp. 201-7, Aug 2003.

[140] L. Moroni, M. Curti, M. Welti, S. Korom, W. Weder, J. R. de Wijn, and C. A. van Blitterswijk, "Anatomical 3d fiber-deposited scaffolds for tissue engineering: designing a neotrachea," Tissue Eng, vol. 13, pp. 2483-93, Oct 2007.

[141] Q. Tan, S. Hillinger, C. A. van Blitterswijk, and W. Weder, "Intra-scaffold continuous medium flow combines chondrocyte seeding and culture systems for tissue engineered trachea construction," Interact Cardiovasc Thorac Surg, vol. 8, pp. 27-30, Jan 2009.

[142] H. Igai, S. S. Chang, M. Gotoh, Y. Yamamoto, M. Yamamoto, Y. Tabata, and $\mathrm{H}$. Yokomise, "Tracheal cartilage regeneration and new bone formation by slow release of bone morphogenetic protein (bmp)-2," ASAIO J, vol. 54, pp. 104-8, Jan 2008.

[143] J. H. Henderson, J. F. Welter, J. M. Mansour, C. Niyibizi, A. I. Caplan, and J. E. Dennis, "Cartilage tissue engineering for laryngotracheal reconstruction: comparison of chondrocytes from three anatomic locations in the rabbit," Tissue Eng, vol. 13, pp. 843-53, Apr 2007. 
[144] M. Weidenbecher, J. H. Henderson, H. M. Tucker, J. Z. Baskin, A. Awadallah, and J. E. Dennis, "Hyaluronan-based scaffolds to tissue-engineer cartilage implants for laryngotracheal reconstruction," Laryngoscope, vol. 117, pp. 1745-9, Oct 2007.

[145] Y. Ni, X. Zhao, L. Zhou, Z. Shao, W. Yan, X. Chen, Z. Cao, Z. Xue, and J. J. Jiang, "Radiologic and histologic characterization of silk fibroin as scaffold coating for rabbit tracheal defect repair," Otolaryngol Head Neck Surg, vol. 139, pp. 256-61, Aug 2008.

[146] P. Macchiarini, P. Jungebluth, T. Go, M. A. Asnaghi, L. E. Rees, T. A. Cogan, A. Dodson, J. Martorell, S. Bellini, P. P. Parnigotto, S. C. Dickinson, A. P. Hollander, S. Mantero, M. T. Conconi, and M. A. Birchall, "Clinical transplantation of a tissue-engineered airway," Lancet, vol. 372, pp. 2023-30, Dec 2008.

[147] A. Seguin, D. Radu, M. Holder-Espinasse, P. Bruneval, A. Fialaire-Legendre, M. Duterque-Coquillaud, A. Carpentier, and E. Martinod, "Tracheal replacement with cryopreserved, decellularized, or glutaraldehyde-treated aortic allografts," Ann Thorac Surg, vol. 87, pp. 861-7, Mar 2009.

[148] P. Macchiarini, T. Walles, C. Biancosino, and H. Mertsching, "First human transplantation of a bioengineered airway tissue," J Thorac Cardiovasc Surg, vol. 128, pp. 63841, Oct 2004.

[149] T. Walles, B. Giere, P. Macchiarini, and H. Mertsching, "Expansion of chondrocytes in a three-dimensional matrix for tracheal tissue engineering," Ann Thorac Surg, vol. 78, pp. 444-8; discussion 448-9, Aug 2004.

[150] T. Walles, B. Giere, M. Hofmann, J. Schanz, F. Hofmann, H. Mertsching, and P. Macchiarini, "Experimental generation of a tissue-engineered functional and vascularized trachea," J Thorac Cardiovasc Surg, vol. 128, pp. 900-6, Dec 2004.

[151] T. W. Gilbert, S. Gilbert, M. Madden, S. D. Reynolds, and S. F. Badylak, "Morphologic assessment of extracellular matrix scaffolds for patch tracheoplasty in a canine model," Ann Thorac Surg, vol. 86, pp. 967-74; discussion 967-74, Sep 2008.

[152] T. Go, P. Jungebluth, S. Baiguero, A. Asnaghi, J. Martorell, H. Ostertag, S. Mantero, M. Birchall, A. Bader, and P. Macchiarini, "Both epithelial cells and mesenchymal stem cell-derived chondrocytes contribute to the survival of tissue-engineered airway transplants in pigs," J Thorac Cardiovasc Surg, vol. 139, pp. 437-43, Feb 2010.

[153] P. Jungebluth, T. Go, A. Asnaghi, S. Bellini, J. Martorell, C. Calore, L. Urbani, H. Ostertag, S. Mantero, M. T. Conconi, and P. Macchiarini, "Structural and morphologic evaluation of a novel detergent-enzymatic tissue-engineered tracheal tubular matrix," J Thorac Cardiovasc Surg, vol. 138, pp. 586-93; discussion 592-3, Sep 2009.

[154] N. T. Remlinger, C. A. Czajka, M. E. Juhas, D. A. Vorp, D. B. Stolz, S. F. Badylak, S. Gilbert, and T. W. Gilbert, "Hydrated xenogeneic decellularized tracheal matrix as a scaffold for tracheal reconstruction," Biomaterials, vol. 31, pp. 3520-6, May 2010. 
[155] Y. Yamamoto, T. Okamoto, M. Goto, H. Yokomise, M. Yamamoto, and Y. Tabata, "Experimental study of bone morphogenetic proteins-2 slow release from an artificial trachea made of biodegradable materials: evaluation of stenting time," ASAIO J, vol. 49, pp. 533-6, Jan 2003.

[156] K. von der Mark, V. Gauss, H. von der Mark, and P. Müller, "Relationship between cell shape and type of collagen synthesised as chondrocytes lose their cartilage phenotype in culture," Nature, vol. 267, pp. 531-2, Jun 1977.

[157] H. C. Grillo, "Development of tracheal surgery: a historical review. part 1: Techniques of tracheal surgery," The Annals of thoracic surgery, vol. 75, pp. 610-9, Feb 2003.

[158] M. Komura, H. Komura, Y. Tanaka, Y. Kanamori, M. Sugiyama, S. Nakahara, H. Kawashima, K. Suzuki, K. Hoshi, and T. Iwanaka, "Human tracheal chondrocytes as a cell source for augmenting stenotic tracheal segments: the first feasibility study in an in vivo culture system," Pediatr Surg Int, vol. 24, pp. 1117-21, Oct 2008.

[159] K. Kojima, L. J. Bonassar, R. A. Ignotz, K. Syed, J. Cortiella, and C. A. Vacanti, "Comparison of tracheal and nasal chondrocytes for tissue engineering of the trachea," Ann Thorac Surg, vol. 76, pp. 1884-8, Dec 2003.

[160] T. Suzuki, K. Kobayashi, Y. Tada, Y. Suzuki, I. Wada, T. Nakamura, and K. Omori, "Regeneration of the trachea using a bioengineered scaffold with adipose-derived stem cells," Ann Otol Rhinol Laryngol, vol. 117, pp. 453-63, Jun 2008.

[161] J. R. Fuchs, D. Hannouche, S. Terada, J. P. Vacanti, and D. O. Fauza, "Fetal tracheal augmentation with cartilage engineered from bone marrow-derived mesenchymal progenitor cells," J Pediatr Surg, vol. 38, pp. 984-7, Jun 2003.

[162] K. Kojima, R. A. Ignotz, T. Kushibiki, K. W. Tinsley, Y. Tabata, and C. A. Vacanti, "Tissue-engineered trachea from sheep marrow stromal cells with transforming growth factor beta2 released from biodegradable microspheres in a nude rat recipient," J Thorac Cardiovasc Surg, vol. 128, pp. 147-53, Jul 2004.

[163] T. Nakamura, T. Sato, M. Araki, S. Ichihara, A. Nakada, M. Yoshitani, S. ichi Itoi, M. Yamashita, S. ichi Kanemaru, K. Omori, Y. Hori, K. Endo, Y. Inada, and K. Hayakawa, "In situ tissue engineering for tracheal reconstruction using a luminar remodeling type of artificial trachea," J Thorac Cardiovasc Surg, vol. 138, pp. 811-9, Oct 2009 .

[164] J. rui Zhang, F. lin Chen, W. Wu, J. hua Wei, X. hua Feng, and T. qiu Mao, "[constructing tissue engineered trachea-like cartilage graft in vitro by using bone marrow stromal cells sheet and plga internal support: experimental study in bioreactor]," Zhonghua Zheng Xing Wai Ke Za Zhi, vol. 25, pp. 124-8, Mar 2009. 
[165] S. M. Kunisaki, D. A. Freedman, and D. O. Fauza, "Fetal tracheal reconstruction with cartilaginous grafts engineered from mesenchymal amniocytes," J Pediatr Surg, vol. 41, pp. 675-82; discussion 675-82, Apr 2006.

[166] G. M. Roomans, "Tissue engineering and the use of stem/progenitor cells for airway epithelium repair," Eur Cell Mater, vol. 19, pp. 284-99, Jan 2010.

[167] C. Coraux, B. Nawrocki-Raby, J. Hinnrasky, C. Kileztky, D. Gaillard, C. Dani, and E. Puchelle, "Embryonic stem cells generate airway epithelial tissue," Am J Respir Cell Mol Biol, vol. 32, pp. 87-92, Feb 2005.

[168] C. Pfenninger, I. Leinhase, M. Endres, N. Rotter, A. Loch, J. Ringe, and M. Sittinger, "Tracheal remodeling: comparison of different composite cultures consisting of human respiratory epithelial cells and human chondrocytes," In Vitro Cell Dev Biol Anim, vol. 43, pp. 28-36, Jan 2007.

[169] E. J. Doolin, L. F. Strande, X. Sheng, and C. W. Hewitt, "Engineering a composite neotrachea with surgical adhesives," J Pediatr Surg, vol. 37, pp. 1034-7, Jul 2002.

[170] K. Kojima, L. J. Bonassar, A. K. Roy, H. Mizuno, J. Cortiella, and C. A. Vacanti, "A composite tissue-engineered trachea using sheep nasal chondrocyte and epithelial cells," FASEB J, vol. 17, pp. 823-8, May 2003.

[171] J. Sakata, C. Vacanti, B. Schloo, G. Healy, R. Langer, and J. P. Vacanti, "Tracheal composites tissue engineered from chondrocytes, tracheal epithelial cells, and synthetic degradable scaffolding," Transplant Proc, vol. 26(6), pp. 3309-3310, Jan 1994.

[172] Y. Goto, Y. Noguchi, A. Nomura, T. Sakamoto, Y. Ishii, S. Bitoh, C. Picton, Y. Fujita, T. Watanabe, S. Hasegawa, and Y. Uchida, "In vitro reconstitution of the tracheal epithelium," Am J Respir Cell Mol Biol, vol. 20, pp. 312-8, Feb 1999.

[173] B. G. Zani, K. Kojima, C. A. Vacanti, and E. R. Edelman, "Tissue-engineered endothelial and epithelial implants differentially and synergistically regulate airway repair," Proc Natl Acad Sci USA, vol. 105, pp. 7046-51, May 2008.

[174] G. Tani, N. Usui, M. Kamiyama, T. Oue, and M. Fukuzawa, "In vitro construction of scaffold-free cylindrical cartilage using cell sheet-based tissue engineering," Pediatr Surg Int, vol. 26, pp. 179-85, Feb 2010.

[175] E. J. O. ten Hallers, G. Rakhorst, H. A. M. Marres, J. A. Jansen, T. G. van Kooten, H. K. Schutte, J.-P. van Loon, E. B. van der Houwen, and G. J. Verkerke, "Animal models for tracheal research," Biomaterials, vol. 25, pp. 1533-43, Apr 2004.

[176] S. H. Kamil, R. D. Eavey, M. P. Vacanti, C. A. Vacanti, and C. J. Hartnick, "Tissueengineered cartilage as a graft source for laryngotracheal reconstruction: a pig model," Arch Otolaryngol Head Neck Surg, vol. 130, pp. 1048-51, Sep 2004. 
[177] M. Weidenbecher, H. M. Tucker, D. A. Gilpin, and J. E. Dennis, "Tissue-engineered trachea for airway reconstruction," Laryngoscope, vol. 119, pp. 2118-23, Nov 2009.

[178] H. C. Ott, T. S. Matthiesen, S. K. Goh, L. D. Black, S. M. Kren, T. I. Netoff, and D. A. Taylor, "Perfusion-decellularized matrix: using nature's platform to engineer a bioartificial heart," Nat. Med., vol. 14, pp. 213-221, Feb 2008.

[179] B. E. Uygun, A. Soto-Gutierrez, H. Yagi, M. L. Izamis, M. A. Guzzardi, C. Shulman, J. Milwid, N. Kobayashi, A. Tilles, F. Berthiaume, M. Hertl, Y. Nahmias, M. L. Yarmush, and K. Uygun, "Organ reengineering through development of a transplantable recellularized liver graft using decellularized liver matrix," Nat. Med., vol. 16, pp. 814-820, Jul 2010.

[180] J. L. Proc, M. A. Kuzyk, D. B. Hardie, J. Yang, D. S. Smith, A. M. Jackson, C. E. Parker, and C. H. Borchers, "A quantitative study of the effects of chaotropic agents, surfactants, and solvents on the digestion efficiency of human plasma proteins by trypsin," J. Proteome Res., vol. 9, pp. 5422-5437, Oct 2010.

[181] L. Du, X. Wu, K. Pang, and Y. Yang, "Histological evaluation and biomechanical characterisation of an acellular porcine cornea scaffold," $\mathrm{Br} J$ Ophthalmol, vol. 95, pp. 410-414, Mar 2011.

[182] B. N. Brown, J. E. Valentin, A. M. Stewart-Akers, G. P. McCabe, and S. F. Badylak, "Macrophage phenotype and remodeling outcomes in response to biologic scaffolds with and without a cellular component," Biomaterials, vol. 30, pp. 1482-1491, Mar 2009.

[183] T. Woods and P. F. Gratzer, "Effectiveness of three extraction techniques in the development of a decellularized bone-anterior cruciate ligament-bone graft," Biomaterials, vol. 26, pp. 7339-7349, Dec 2005.

[184] T. W. Hudson, S. Y. Liu, and C. E. Schmidt, "Engineering an improved acellular nerve graft via optimized chemical processing," Tissue Eng., vol. 10, no. 9-10, pp. 1346-1358, 2004.

[185] J. Hodde and M. Hiles, "Virus safety of a porcine-derived medical device: evaluation of a viral inactivation method," Biotechnol. Bioeng., vol. 79, pp. 211-216, Jul 2002.

[186] J. Hodde, A. Janis, D. Ernst, D. Zopf, D. Sherman, and C. Johnson, "Effects of sterilization on an extracellular matrix scaffold: part I. Composition and matrix architecture," J Mater Sci Mater Med, vol. 18, pp. 537-543, Apr 2007.

[187] T. W. Gilbert, S. Wognum, E. M. Joyce, D. O. Freytes, M. S. Sacks, and S. F. Badylak, "Collagen fiber alignment and biaxial mechanical behavior of porcine urinary bladder derived extracellular matrix," Biomaterials, vol. 29, pp. 4775-4782, Dec 2008. 
[188] L. Santelices, S. Calano, J. Erhart, R. L. Prantil, J. L. Haney, D. A. Vorp, and J. M. Ahearn, "Experimental system for ex vivo measurement of murine aortic stiffness," Physiol Meas, vol. 28(8), p. N39, Jan 2007.

[189] S. F. Badylak, D. A. Vorp, A. R. Spievack, A. Simmons-Byrd, J. Hanke, D. O. Freytes, A. Thapa, T. W. Gilbert, and A. Nieponice, "Esophageal reconstruction with ecm and muscle tissue in a dog model," Journal of Surgical ..., vol. 128(1), pp. 87-97, Jan 2005.

[190] R. J. Jankowski, R. L. Prantil, M. O. Fraser, M. B. Chancellor, W. C. de Groat, J. Huard, and D. A. Vorp, "Development of an experimental system for the study of urethral biomechanical function," Am J Physiol, vol. 286, pp. F225-F232, Jan 2004.

[191] T. Gibson, "The long term survival of cartilage homografts in man," Br J Plast Surg, vol. 11, pp. 177-87, 1958.

[192] P. P. Aubin, H. K. Cheah, A. M. Davis, and A. E. Gross, "Long-term followup of fresh femoral osteochondral allografts for posttraumatic knee defects," Clin. Orthop. Relat. Res., pp. S318-327, Oct 2001.

[193] P. Whiteman, "The quantitative measurement of Alcian Blue-glycosaminoglycan complexes," Biochem. J., vol. 131, pp. 343-350, Feb 1973.

[194] Y. Wang, Y. C. Huang, A. A. Gertzman, L. Xie, A. Nizkorodov, S. L. Hyzy, K. Truncale, R. E. Guldberg, Z. Schwartz, and B. D. Boyan, "Endogenous regeneration of critical-size chondral defects in immunocompromised rat xiphoid cartilage using decellularized human bone matrix scaffolds," Tissue Eng Part A, vol. 18, pp. 2332-2342, Nov 2012.

[195] B. D. Elder, S. V. Eleswarapu, and K. A. Athanasiou, "Extraction techniques for the decellularization of tissue engineered articular cartilage constructs," Biomaterials, vol. 30, pp. 3749-3756, Aug 2009.

[196] W. Q. Sun and P. Leung, "Calorimetric study of extracellular tissue matrix degradation and instability after gamma irradiation," Acta Biomater, vol. 4, pp. 817-826, Jul 2008.

[197] V. Samouillan, F. Delaunay, J. Dandurand, N. Merbahi, J.-P. Gardou, M. Yousfi, A. Gandaglia, M. Spina, and C. Lacabanne, "The use of thermal techniques for the characterization and selection of natural biomaterials," Journal of Functional Biomaterials, vol. 2, no. 3, pp. 230-248, 2011.

[198] G. S. Kassab, "Biomechanics of the cardiovascular system: the aorta as an illustratory example," J R Soc Interface, vol. 3, pp. 719-740, Dec 2006.

[199] S. Haykal, Y. Zhou, P. Marcus, M. Salna, T. Machuca, S. O. Hofer, and T. K. Waddell, "The effect of decellularization of tracheal allografts on leukocyte infiltration and of 
recellularization on regulatory T cell recruitment," Biomaterials, vol. 34, pp. 58215832, Jul 2013.

[200] M. Zang, Q. Zhang, E. I. Chang, A. B. Mathur, and P. Yu, "Decellularized tracheal matrix scaffold for tracheal tissue engineering: in vivo host response," Plast. Reconstr. Surg., vol. 132, pp. 549e-559e, Oct 2013.

[201] T. W. Gilbert, T. L. Sellaro, and S. F. Badylak, "Decellularization of tissues and organs," Biomaterials, vol. 27, pp. 3675-3683, Jul 2006.

[202] M. V. Kisilevski, N. I. u. Anisimova, O. V. Lebedinskaia, B. E. Polotski, and M. I. Davydov, "Heterotopic transplantation of non-immunogenic trachea populated with recipient bone marrow stromal cells," Morfologiia, vol. 141, no. 1, pp. 66-70, 2012.

[203] M. J. Elliott, P. D. Coppi, S. Speggiorin, D. Roebuck, C. R. Butler, E. Samuel, C. Crowley, C. McLaren, A. Fierens, D. Vondrys, L. Cochrane, C. Jephson, S. Janes, N. J. Beaumont, T. Cogan, A. Bader, A. M. Seifalian, J. J. Hsuan, M. W. Lowdell, and M. A. Birchall, "Stem-cell-based, tissue engineered tracheal replacement in a child: a 2-year follow-up study," The Lancet, vol. 380, no. 9846, pp. 994 - 1000, 2012.

[204] C. Carruthers, Tissue and Whole Organ Decellularization: An Evaluation of Cytocompatibility and Mechanics. Doctoral Dissertation, University of Pittsburgh, 2014.

[205] B. N. Brown, B. D. Ratner, S. B. Goodman, S. Amar, and S. F. Badylak, "Macrophage polarization: an opportunity for improved outcomes in biomaterials and regenerative medicine," Biomaterials, vol. 33, pp. 3792-3802, May 2012.

[206] B. N. Brown, R. Londono, S. Tottey, L. Zhang, K. A. Kukla, M. T. Wolf, K. A. Daly, J. E. Reing, and S. F. Badylak, "Macrophage phenotype as a predictor of constructive remodeling following the implantation of biologically derived surgical mesh materials," Acta Biomater, vol. 8, pp. 978-987, Mar 2012.

[207] T. J. Keane, R. Londono, N. J. Turner, and S. F. Badylak, "Consequences of ineffective decellularization of biologic scaffolds on the host response," Biomaterials, vol. 33, pp. 1771-1781, Feb 2012.

[208] D. Faulk, S. Johnson, and S. Badylak, "7 - decellularized biological scaffolds for cardiac repair and regeneration," in Cardiac Regeneration and Repair (R.-K. Li and R. D. Weisel, eds.), pp. 180 - 200, Woodhead Publishing, 2014.

[209] C. Y. Woon, B. C. Pridgen, A. Kraus, S. Bari, H. Pham, and J. Chang, "Optimization of human tendon tissue engineering: peracetic acid oxidation for enhanced reseeding of acellularized intrasynovial tendon," Plast. Reconstr. Surg., vol. 127, pp. 1107-1117, Mar 2011. 
[210] K. Daly, S. Liu, V. Agrawal, B. Brown, S. Johnson, C. Medberry, and S. Badylak, "Damage associated molecular patterns within xenogeneic biologic scaffolds and their effects on host remodeling," Biomaterials, vol. 33, no. 1, pp. 91 - 101, 2012.

[211] J. Hussong, R. Lindken, P. Faulhammer, K. Noreikat, K. Sharp, W. Kummer, and J. Westerweel, "Cilia-driven particle and fluid transport over mucus-free mice tracheae," Journal of Biomechanics, vol. 46, no. 3, pp. 593 - 598, 2013.

[212] J. C. Kutten, D. McGovern, C. M. Hobson, S. A. Luffy, A. Nieponice, K. Tobita, R. J. Francis, S. D. Reynolds, J. S. Isenberg, and T. W. Gilbert, "Decellularized Tracheal Extracellular Matrix Supports Epithelial Migration, Differentiation, and Function.," Tissue engineering. Part A, Sep 2014.

[213] G. Abouna, "Organ shortage crisis: Problems and possible solutions," Transplantation Proceedings, vol. 40, no. 1, pp. $34-38,2008$.

[214] A. R. Kolker, D. J. Brown, J. S. Redstone, V. M. Scarpinato, and M. K. Wallack, "Multilayer reconstruction of abdominal wall defects with acellular dermal allograft (AlloDerm) and component separation," Ann Plast Surg, vol. 55, pp. 36-41, Jul 2005.

[215] D. J. Wainwright, "Use of an acellular allograft dermal matrix (AlloDerm) in the management of full-thickness burns," Burns, vol. 21, pp. 243-248, Jun 1995.

[216] M. J. Sawkins, W. Bowen, P. Dhadda, H. Markides, L. E. Sidney, A. J. Taylor, F. R. Rose, S. F. Badylak, K. M. Shakesheff, and L. J. White, "Hydrogels derived from demineralized and decellularized bone extracellular matrix," Acta Biomater, vol. 9, pp. 7865-7873, Aug 2013.

[217] M. S. Lee, "GraftJacket augmentation of chronic Achilles tendon ruptures," Orthopedics, vol. 27, pp. s151-153, Jan 2004.

[218] S. F. Badylak, "Xenogeneic extracellular matrix as a scaffold for tissue reconstruction," Transpl. Immunol., vol. 12, pp. 367-377, Apr 2004.

[219] S. F. Badylak, D. O. Freytes, and T. W. Gilbert, "Extracellular matrix as a biological scaffold material: Structure and function," Acta Biomaterialia, vol. 5, no. 1, pp. 1 13, 2009.

[220] J. Negishi, S. Funamoto, T. Kimura, K. Nam, T. Higami, and A. Kishida, "Effect of treatment temperature on collagen structures of the decellularized carotid artery using high hydrostatic pressure," Journal of Artificial Organs, vol. 14, no. 3, pp. 223-231, 2011.

[221] K. Sawada, D. Terada, T. Yamaoka, S. Kitamura, and T. Fujisato, "Cell removal with supercritical carbon dioxide for acellular artificial tissue," Journal of Chemical Technology \& Biotechnology, vol. 83, no. 6, pp. 943-949, 2008. 
[222] J. E. Reing, B. N. Brown, K. A. Daly, J. M. Freund, T. W. Gilbert, S. X. Hsiong, A. Huber, K. E. Kullas, S. Tottey, M. T. Wolf, and S. F. Badylak, "The effects of processing methods upon mechanical and biologic properties of porcine dermal extracellular matrix scaffolds," Biomaterials, vol. 31, pp. 8626-8633, Nov 2010.

[223] R. W. Chan and N. Tayama, "Biomechanical effects of hydration in vocal fold tissues," Otolaryngology - Head and Neck Surgery, vol. 126, no. 5, pp. 528 - 537, 2002.

[224] J. Liao, E. M. Joyce, and M. S. Sacks, "Effects of decellularization on the mechanical and structural properties of the porcine aortic valve leaflet," Biomaterials, vol. 29, pp. 1065-1074, Mar 2008.

[225] Y. Zou and Y. Zhang, "Mechanical evaluation of decellularized porcine thoracic aorta," J. Surg. Res., vol. 175, pp. 359-368, Jun 2012.

[226] M. H. Lavietes, S. C. Smeltzer, S. D. Cook, R. M. Modak, and G. C. Smaldone, "Airway dynamics, oesophageal pressure and cough," Eur. Respir. J., vol. 11, pp. 156-161, Jan 1998. 